Exact computation of one-loop correction to the energy of folded spinning string in $\operatorname{AdS}_{5} \times S^{5}$

This content has been downloaded from IOPscience. Please scroll down to see the full text.

2010 J. Phys. A: Math. Theor. 43165402

(http://iopscience.iop.org/1751-8121/43/16/165402)

View the table of contents for this issue, or go to the journal homepage for more

Download details:

IP Address: 194.94.224.254

This content was downloaded on 15/01/2015 at 11:35

Please note that terms and conditions apply. 


\title{
Exact computation of one-loop correction to the energy of folded spinning string in $\operatorname{AdS}_{5} \times S^{5}$
}

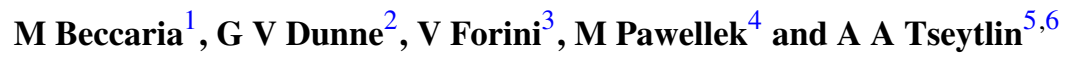 \\ ${ }^{1}$ Physics Department, Salento University and INFN, 73100 Lecce, Italy \\ 2 Department of Physics, University of Connecticut, Storrs CT 06269-3046, USA \\ ${ }^{3}$ Max-Planck-Institut für Gravitationsphysik, Albert-Einstein-Institut Am Mühlenberg 1, \\ D-14476 Potsdam, Germany \\ ${ }^{4}$ Institut für Theoretische Physik III, Universität Erlangen-Nürnberg, Staudtstr.7, \\ D-91058 Erlangen, Germany \\ 5 The Blackett Laboratory, Imperial College, London SW7 2AZ, UK \\ 6 Also at Lebedev Institute, Moscow \\ E-mail: beccaria@le.infn.it, dunne@phys.uconn.edu, forini@aei.mpg.de, \\ Michael.Pawellek@physik.uni-erlangen.de and tseytlin@imperial.ac.uk
}

Received 3 February 2010

Published 31 March 2010

Online at stacks.iop.org/JPhysA/43/165402

\begin{abstract}
We consider the one-loop correction to the energy of the folded spinning string solution in the $\mathrm{AdS}_{3}$ part of $\mathrm{AdS}_{5} \times S^{5}$. The classical string solution is expressed in terms of elliptic functions so an explicit computation of the corresponding fluctuation determinants for generic values of the spin appears to be a nontrivial problem. We show how it can be solved exactly by using the staticgauge expression for the string partition function (which we demonstrate to be equivalent to the conformal gauge one) and observing that all the corresponding second-order fluctuation operators can be put into the standard (single-gap) Lamé form. We systematically derive the small-spin and large-spin expansions of the resulting expression for the string energy and comment on some of their applications.
\end{abstract}

PACS numbers: 11.25.Tq, 11.25.-w, 11.15.Kc

(Some figures in this article are in colour only in the electronic version)

\section{Introduction}

Classical string solutions in $\mathrm{AdS}_{5} \times S^{5}$, or non-topological solitons of the string sigma model, play an important guiding role in the study of gauge-string duality. One of the basic examples is the folded spinning string in AdS [1,2]. The classical string energy is a non-trivial function of the spin, interpolating between the flat-space regime, $E \sim \sqrt{S}+\cdots$, for small spin and the 
scaling AdS regime, $E=S+a \ln S+\cdots$, for large spin. The dual gauge-theory interpretation of the latter was suggested in [1] and since then was used and verified in many papers.

The form of this spinning string solution is determined by an elliptic sn function (a solution of the sinh-Gordon equation). Computing the quantum correction to its energy in $\mathrm{AdS}_{5} \times S^{5}$ string theory is thus a non-trivial problem, first addressed in [3]. In [3] the one-loop correction to the energy $E_{1}(S)$ was expressed in terms of the determinants of the bosonic and fermionic fluctuation operators with elliptic-function potentials. It was explicitly computed only in the large-spin limit when the solution simplifies drastically (elliptic-function potentials become constant). Recently, attempts were made to compute the first few leading terms in $E_{1}(S)$ in the small $S$ [9] and the large $S$ [10] expansions.

The aim of the present paper is to solve the problem addressed in [3], i.e. to present the general analytic expression for the one-loop correction $E_{1}(S)$ for an arbitrary value of the spin. This would enable us to systematically expand $E_{1}$ in the small $S$ or large $S$ limits.

The study of the large $S$ expansion of string energy is important for several reasons, e.g. (i) for comparison with the Bethe ansatz predictions (see, e.g. [4-7]); (ii) for further verification of the reciprocity property at strong coupling $[8,10]$; (iii) for understanding the on-set of finite size (exponential or 'wrapping') corrections in the anomalous dimension of the corresponding twist two-gauge-theory operator (cf [11-13]) and the problem of orders of large-spin/large-coupling limits. The study of the small $S$ expansion may shed light on quantum corrections to quantum string states or 'short' operators [9, 14].

It is also interesting to compare the explicit form of the one-loop string correction derived directly from the string theory action with the expression coming out of the approach based on classical integrability of the string sigma model $[15,16]$. The two should match in general (see [17] and references therein) but detailed comparison may teach us important lessons about the workings of the integrability in the case of cylindrical world-sheet topology.

We shall start in section 2 with a summary of the basic relations for the classical spinning string solution of $[1,2]$. Then in section 3 we shall review the approach of [3] to the computation of the one-loop correction $E_{1}$ to the string energy. In addition to having ellipticfunction potentials in the quadratic fluctuation operators, a complication of the conformalgauge expression for the one-loop partition function of the string sigma model expanded near this solution is a mixing of the three $\mathrm{AdS}_{3}$ modes. This mixing is absent in the static gauge [3], and we go beyond the discussion in [3] by arguing that the conformal-gauge and the staticgauge expressions are indeed equivalent (in particular, the string correction in the static gauge is also UV finite). This allows us to use the static-gauge expression for $E_{1}$ in which all $8+8$ bosonic and fermionic fluctuation modes are decoupled as a starting point of our investigation.

A further crucial observation made in section 3.3 is that all second-order fluctuation operators in this stationary soliton problem can be put into the standard single-gap Lamé ordinary differential operator form on a circle. As discussed in section 4, this allows us to compute their determinants in an explicit way. In section 4.1 we review several equivalent forms of the general expression for the determinant of the second-order ordinary differential operator $\mathcal{O}=-\partial_{x}^{2}+V(x)$ on a circle: in terms of the discriminant, in terms of the quasimomentum and in terms of the $\zeta$-function or resolvent. In section 4.2 we specify these relations to the case of the Lamé potential $V=2 k^{2} \operatorname{sn}^{2}\left(x \mid k^{2}\right)$. Then in section 4.3 we apply this formalism to the case of the fluctuation operators whose determinants appear in the string one-loop correction $E_{1}$.

In section 5 we first demonstrate that the resulting expression for $E_{1}$ is UV finite as expected. We then check the equivalence between the conformal-gauge and the static-gauge expressions for $E_{1}$ by numerically evaluating the 'mixed' conformal-gauge determinant and comparing it with its static-gauge counterpart that we found analytically. We also plot $E_{1}$ and 
compare it with its large-spin and small-spin asymptotics derived analytically in sections 6 and 7. In section 6 we also check the reciprocity constraints on the few leading terms in the large-spin expansion of the energy.

Some concluding remarks are made in section 8. One natural extension that we plan to address in the future [18] is to repeat the analysis of the present paper in the case of the $(S, J)$ folded string solution with an extra orbital momentum in $S^{5}$ [3]. This problem is more complicated in that even the static-gauge expression for the fluctuation Lagrangian has now two mixed fluctuations and thus the standard expressions for the Lamé operator determinant cannot be directly applied.

There are several appendices containing notation and technical details. In appendix A we summarize the basic definitions for the elliptic functions, and describe the Landen transformation used to convert certain fluctuation operators to the Lamé form. Appendix B describes the Gel'fand-Yaglom numerical method for computing determinants of secondorder differential operators, including the case of coupled operators. Appendix C contains the details of the relevant elliptic-function expansions needed for studying the small-spin and the large-spin limits, while in appendix D we evaluate the leading correction due to the (exponentially suppressed) contributions that we neglect in the main calculation. In appendix E we consider an alternative approach to the expansion of the one-loop energy in the large-spin limit. Appendix F relates our exact results to the perturbative expansion of the associated determinants.

\section{Review of the folded spinning string solution in $\mathbf{A d S}_{3}$}

The folded spinning string in $\mathrm{AdS}_{3}$ space

$$
\mathrm{d} s^{2}=-\cosh ^{2} \rho \mathrm{d} t^{2}+\mathrm{d} \rho^{2}+\sinh ^{2} \rho \mathrm{d} \phi^{2}
$$

is a classical closed string solution given by [1]

$$
t=\kappa \tau, \quad \phi=\omega \tau, \quad \rho=\rho(\sigma)=\rho(\sigma+2 \pi),
$$

where $\kappa, \omega$ are the constant parameters. The equation of motion in conformal gauge ${ }^{7}$ and its solution with initial condition $\rho(0)=0$ are $^{8}$

$\rho^{\prime 2}=\kappa^{2} \cosh ^{2} \rho-w^{2} \sinh ^{2} \rho$

$\sinh \rho(\sigma)=\frac{k}{\sqrt{1-k^{2}}} \operatorname{cn}\left(\omega \sigma+\mathbb{K} \mid k^{2}\right), \quad \rho^{\prime}(\sigma)=\kappa \operatorname{sn}\left(\omega \sigma+\mathbb{K} \mid k^{2}\right)$,

where $\mathbb{K} \equiv \mathbb{K}\left(k^{2}\right)$ is the complete elliptic integral of the first kind [30], with elliptic modulus given by $k \equiv \frac{\kappa}{\omega} \cdot{ }^{9}$ Here $\rho$ varies from 0 to its maximal value $\rho_{0}$, which is related to the useful parameter $\eta$ or $k$ by

$$
\operatorname{coth}^{2} \rho_{0}=\frac{\omega^{2}}{\kappa^{2}} \equiv 1+\eta \equiv \frac{1}{k^{2}} \text {. }
$$

The periodicity implies an extra condition for the parameters

$$
2 \pi=\int_{0}^{2 \pi} \mathrm{d} \sigma=4 \int_{0}^{\rho_{0}} \frac{\mathrm{d} \rho}{\sqrt{\kappa^{2} \cosh ^{2} \rho-\omega^{2} \sinh ^{2} \rho}}
$$

7 We use Minkowski signature in both target space and world sheet, so that in conformal gauge $\sqrt{-g} g^{a b}=\eta^{a b}=$ $\operatorname{diag}(-1,1)$.

8 To construct the full ( $2 \pi$ periodic) folded closed string solution one should glue together four such functions $\rho(\sigma)$ on $\frac{\pi}{2}$ intervals and cover the full $0 \leqslant \sigma \leqslant 2 \pi$ interval.

9 See appendix A for notation. We adopt here the Abramowitz-Mathematica notation for the modulus of the elliptic functions. 
integrating which one finds (see (2.5))

$$
\kappa=\frac{2 k}{\pi} \mathbb{K}, \quad \omega=\frac{2}{\pi} \mathbb{K} .
$$

The corresponding induced 2D metric on the $(\tau, \sigma)$ cylinder and its curvature are

$$
g_{a b}=\rho^{\prime 2}(\sigma) \eta_{a b}, \quad R^{(2)}=-\frac{\partial_{\sigma}^{2} \ln \rho^{\prime 2}}{\rho^{\prime 2}}=-2+\frac{2 \kappa^{2} \omega^{2}}{\rho^{\prime 4}} .
$$

The two conserved momenta conjugate to $t$ and $\phi$ are the classical energy and the spin:

$E_{0}=\sqrt{\lambda} \kappa \int_{0}^{2 \pi} \frac{\mathrm{d} \sigma}{2 \pi} \cosh ^{2} \rho \equiv \sqrt{\lambda} \mathcal{E}, \quad S=\sqrt{\lambda} \omega \int_{0}^{2 \pi} \frac{\mathrm{d} \sigma}{2 \pi} \sinh ^{2} \rho \equiv \sqrt{\lambda} \mathcal{S}$.

Using (2.3) and (2.6) we get the following explicit expressions in terms of the complete elliptic integrals $\mathbb{K}=\mathbb{K}\left(k^{2}\right)$ and $\mathbb{E}=\mathbb{E}\left(k^{2}\right)$ (see appendix A):

$$
\begin{aligned}
& \mathcal{E}_{0}=\frac{2}{\pi} \frac{k}{1-k^{2}} \mathbb{E}, \\
& \mathcal{S}=\frac{2}{\pi}\left(\frac{1}{1-k^{2}} \mathbb{E}-\mathbb{K}\right) .
\end{aligned}
$$

To find the energy in terms of the spin, one is to solve for $k$ (or $\eta$ ) in terms of $\mathcal{S}$ and then substitute it into the expression for the energy $\mathcal{E}$. This can be easily done in the following two limiting cases:

(i) large-spin or long-string limit: $\rho_{0} \rightarrow \infty$, i.e. $\eta \rightarrow 0$ or $k \rightarrow 1$

(ii) small-spin or short-string limit: $\rho_{0} \rightarrow 0$, i.e. $\eta \rightarrow \infty$ or $k \rightarrow 0$

In the 'long-string' limit when the string's ends are close to the boundary of $\mathrm{AdS}_{5}$, the spin is automatically large and the parameter $\eta$ is expanded around zero as

$$
\eta=\frac{2}{\mathcal{S}}-\frac{\ln (8 \pi \mathcal{S})-3}{\pi^{2} \mathcal{S}^{2}}+\cdots, \quad \eta \ll 1 .
$$

Substituting this in (2.10) one obtains for the energy the well-known logarithmic behaviour $[1,3,10]$ :

$$
\mathcal{E}_{0}=\mathcal{S}+\frac{\ln (8 \pi \mathcal{S})-1}{\pi}+\frac{\ln (8 \pi \mathcal{S})-1}{2 \pi^{2} \mathcal{S}}+\cdots, \quad \mathcal{S} \gg 1,
$$

where the leading $\ln \mathcal{S}$ term is governed by the so-called scaling function (cusp anomaly) and the subleading ones can be shown to obey non-trivial reciprocity relations $[8,10]$.

In the 'short-string' limit, when the string is rotating in the small central $(\rho=0)$ region of $\mathrm{AdS}_{3}$, the spin is small and the parameter $\eta$ is large

$$
\frac{1}{\eta}=2 \mathcal{S}-\frac{1}{2} \mathcal{S}^{2}+\frac{7}{8} \mathcal{S}^{3}-\frac{117}{64} \mathcal{S}^{4}+\cdots, \quad \eta \gg 1 .
$$

This results in the usual flat-space Regge relation [1, 3, 9]

$$
\mathcal{E}_{0}=\sqrt{2 \mathcal{S}}\left(1+\frac{3}{8} \mathcal{S}+\cdots\right), \quad \mathcal{S} \ll 1
$$

These small- and large-spin expansions of the classical energy $\mathcal{E}_{0}$ are shown in figure 1 , compared to the exact relation. A similar plot for the one-loop correction is provided below, see figure 8 . 


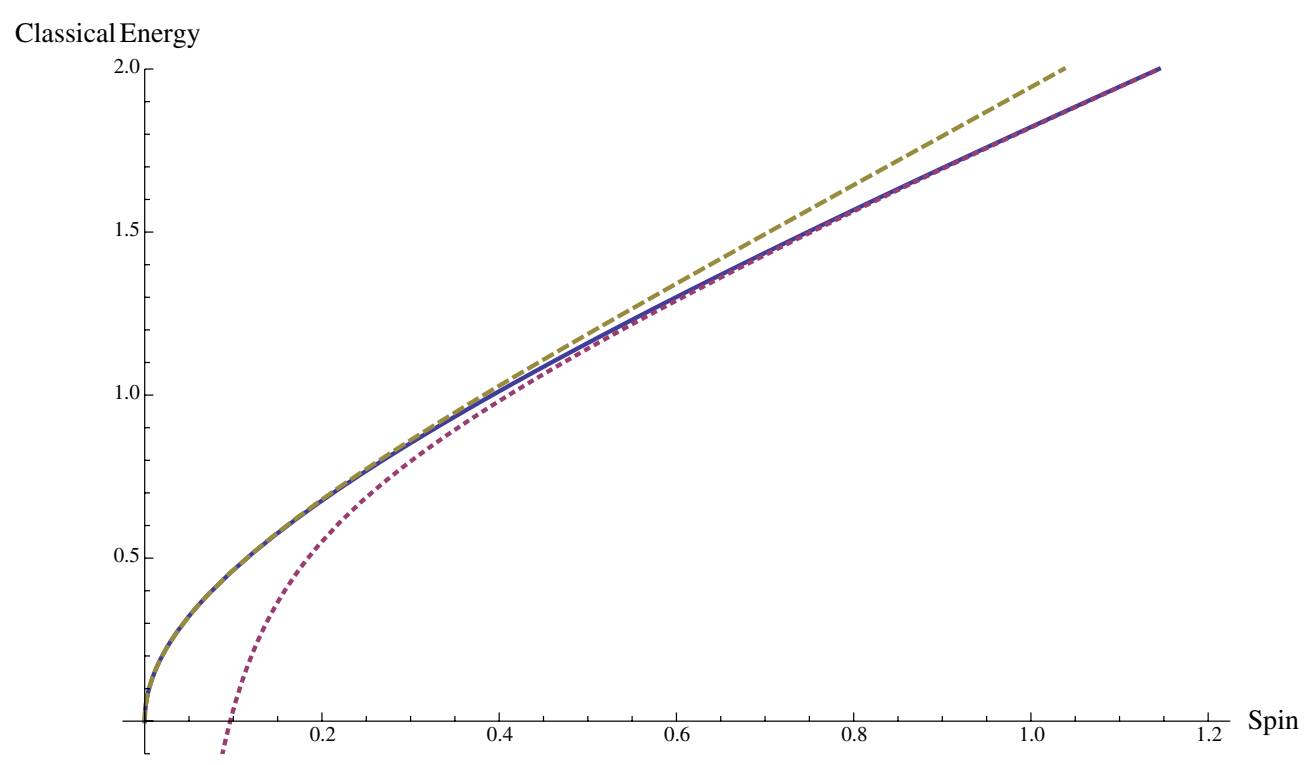

Figure 1. Plot (blue, solid curve) of the classical energy $\mathcal{E}_{0}$ as a function of the spin $\mathcal{S}$, compared with the large-spin expansion (red, dotted curve) in (2.13), and the small-spin expansion (gold, dashed curve) in (2.15).

\section{One-loop correction to the spinning string energy}

As discussed in [3], one can compute the leading quantum correction to the energy of this solution by expanding the action to quadratic order in fluctuations near the classical solution

$$
\widetilde{I}=-\frac{\sqrt{\lambda}}{4 \pi} \int \mathrm{d} \tau \int_{0}^{2 \pi} \mathrm{d} \sigma\left(\widetilde{\mathcal{L}}_{B}+\widetilde{\mathcal{L}}_{F}\right)
$$

and computing the corresponding partition function expressed in terms of determinants of the quadratic fluctuation operators. Then (switching to the Euclidean time $\tau \rightarrow \mathrm{i} \tau$ ) the one-loop correction to the energy can be found from the $2 \mathrm{D}$ effective action $\Gamma$ by dividing over the time interval $(t=\kappa \tau)$

$$
E_{1}=\frac{\Gamma}{\kappa \mathcal{T}}, \quad \mathcal{T} \equiv \int \mathrm{d} \tau \rightarrow \infty, \quad \Gamma=-\ln Z
$$

where $Z$ is given by the ratio of the fermionic and bosonic determinants.

Since the above rigid spinning string solution is stationary, the coefficients in the fluctuation Lagrangian do not depend on $\tau$. Then the relevant 2D functional determinants may be reduced to $1 \mathrm{D}$ determinants as in

$\ln \operatorname{det}\left[-\partial_{\sigma}^{2}-\partial_{\tau}^{2}+M^{2}(\sigma)\right]=\mathcal{T} \int_{-\infty}^{+\infty} \frac{\mathrm{d} \Omega}{2 \pi} \ln \operatorname{det}\left[-\partial_{\sigma}^{2}+\Omega^{2}+M^{2}(\sigma)\right]$,

i.e. we may introduce $\tilde{\Gamma}$ defined by

$$
\Gamma=\mathcal{T} \int_{-\infty}^{+\infty} \frac{\mathrm{d} \Omega}{2 \pi} \tilde{\Gamma}
$$




\subsection{Conformal gauge}

Following [3] one may use either the conformal gauge or the static gauge to compute the fluctuation Lagrangian and thus the corresponding one-loop partition function. The bosonic fluctuation Lagrangian reads

$$
\begin{aligned}
& \tilde{\mathcal{L}}_{B}^{\text {(conf) }}=-\partial_{a} \tilde{t} \partial^{a} \tilde{t}-\mu_{t}^{2} \tilde{t}^{2}+\partial_{a} \tilde{\phi} \partial^{a} \tilde{\phi}+\mu_{\phi}^{2} \tilde{\phi}^{2}+\partial_{a} \tilde{\rho} \partial^{a} \tilde{\rho}+\mu_{\rho}^{2} \tilde{\rho}^{2} \\
& +4 \tilde{\rho}\left(\kappa \sinh \rho \partial_{0} \tilde{t}-\omega \cosh \rho \partial_{0} \tilde{\phi}\right)+\partial_{a} \beta_{u} \partial^{a} \beta_{u}+\mu_{\beta}^{2} \beta_{u}^{2}+\partial_{a} \zeta_{s} \partial^{a} \zeta_{s}, \\
& \mu_{t}^{2}=2 \rho^{\prime 2}-\kappa^{2}, \quad \mu_{\phi}^{2}=2 \rho^{\prime 2}-\omega^{2}, \quad \mu_{\rho}^{2}=2 \rho^{\prime 2}-\omega^{2}-\kappa^{2}, \quad \mu_{\beta}^{2}=2 \rho^{\prime 2} .
\end{aligned}
$$

Here $\beta_{u}(u=1,2)$ are the two $\mathrm{AdS}_{5}$ fluctuations transverse to the $\mathrm{AdS}_{3}$ subspace in which the string is moving, while $\zeta_{s}(s=1, \ldots, 5)$ are fluctuations in $S^{5}$. The three $\operatorname{AdS}_{3}$ fields $(\tilde{t}, \tilde{\rho}, \tilde{\phi})$ are coupled so that the corresponding 1D determinant in (3.3) will involve the following $3 \times 3$ matrix differential operator acting on the three fields $X=(\tilde{t}, \tilde{\rho}, \tilde{\phi})\left(\operatorname{after} \tau \rightarrow \mathrm{i} \tau, \partial_{\tau} \rightarrow \mathrm{i} \Omega\right)$ :

$\mathcal{O}_{t \rho \phi}=\left(\begin{array}{ccc}\partial_{\sigma}^{2}-\Omega^{2}-2 \rho^{\prime 2}+\kappa^{2} & 2 \Omega \kappa \sinh \rho & 0 \\ -2 \Omega \kappa \sinh \rho & -\partial_{\sigma}^{2}+\Omega^{2}+2 \rho^{\prime 2}-\omega^{2} & 2 \Omega \omega \cosh \rho \\ 0 & -2 \Omega \omega \cosh \rho & -\partial_{\sigma}^{2}+\Omega^{2}+2 \rho^{\prime 2}-\omega^{2}-\kappa^{2}\end{array}\right)$.

In addition to the coefficients being dependent on $\sigma$ according to (2.4), this mixing makes finding the determinant of this operator a non-trivial problem. Taking into account the contribution of the two massless conformal-gauge ghosts, the bosonic contribution to $\tilde{\Gamma}=\tilde{\Gamma}_{B}+\tilde{\Gamma}_{F}$ in (3.4) may then be written as

$$
\begin{aligned}
& \tilde{\Gamma}_{B}^{\text {(conf) }}=\frac{1}{2}\left(\ln \operatorname{det} \mathcal{O}_{t \rho \phi}+2 \ln \operatorname{det} \mathcal{O}_{\beta}+3 \ln \operatorname{det} \mathcal{O}_{0}\right), \\
& \mathcal{O}_{\beta}=-\partial_{\sigma}^{2}+\Omega^{2}+2 \rho^{\prime 2}, \quad \mathcal{O}_{0}=-\partial_{\sigma}^{2}+\Omega^{2} .
\end{aligned}
$$

The fermionic part of the quadratic fluctuation Lagrangian can be put into the form [3]

$$
\tilde{\mathcal{L}}_{F}=2 \mathrm{i}\left(\bar{\Psi} \gamma^{a} \partial_{a} \Psi-\mathrm{i} \mu_{F} \bar{\Psi} \gamma_{3} \Psi\right), \quad \mu_{F}=\rho^{\prime},
$$

where $\gamma_{a}$ are $2 \mathrm{D}$ gamma matrices (times a unit $8 \times 8$ matrix) and $\gamma_{3}=\operatorname{diag}(I,-I)$. It may be interpreted as describing a system of 4+4 2D Majorana fermions with $\sigma$-dependent masses $\pm \rho^{\prime}$. Squaring the corresponding Dirac operator, the fermionic contribution to the $2 \mathrm{D}$ effective action $\tilde{\Gamma}$ in (3.4) can be written as (see also [9])

$$
\begin{aligned}
& \tilde{\Gamma}_{F}=-\frac{1}{2}\left(4 \ln \operatorname{det} \mathcal{O}_{\psi_{+}}+4 \ln \operatorname{det} \mathcal{O}_{\psi_{-}}\right), \\
& \mathcal{O}_{\psi_{ \pm}} \equiv-\partial_{\sigma}^{2}+\Omega^{2}+\mu_{\psi_{ \pm}}^{2}, \quad \mu_{\psi_{ \pm}}^{2}= \pm \mu_{F}^{\prime}+\mu_{F}^{2}= \pm \rho^{\prime \prime}+\rho^{\prime 2} .
\end{aligned}
$$

\subsection{Static gauge}

Another approach considered in [3] was to start with the Nambu action and use the same classical solution but impose the static gauge on quantum fluctuations: $\tilde{t}=\tilde{\rho}=0 .{ }^{10}$ In this case the remaining $\mathrm{AdS}_{3}$ mode $\tilde{\phi}$ is decoupled and is described by

$$
\tilde{\mathcal{L}}_{\phi}^{(\text {stat })}=\partial_{a} \tilde{\phi} \partial^{a} \tilde{\phi}+\bar{\mu}_{\phi}^{2} \tilde{\phi}^{2}
$$

${ }^{10}$ The classical solution of section 2 is also the solution of the Nambu action as the induced metric is conformally flat. We may define the static gauge by the condition that $\tau$ and $\sigma$ are such that $t$ and $\rho$ have their classical values, i.e. do not fluctuate. 


$$
\bar{\mu}_{\phi}^{2}=2 \rho^{\prime 2}+\frac{2 \kappa^{2} \omega^{2}}{\rho^{\prime 2}} .
$$

Then the static-gauge analogue of (3.8) takes the form (the masses of other modes are the same as in the conformal gauge but there is no ghost determinant contribution)

$$
\begin{aligned}
& \tilde{\Gamma}_{B}^{\text {(stat) }}=\frac{1}{2}\left(\ln \operatorname{det} \mathcal{O}_{\phi}+2 \ln \operatorname{det} \mathcal{O}_{\beta}+5 \ln \operatorname{det} \mathcal{O}_{0}\right), \\
& \mathcal{O}_{\phi}=-\partial_{\sigma}^{2}+\Omega^{2}+2 \rho^{\prime 2}+\frac{2 \kappa^{2} \omega^{2}}{\rho^{\prime 2}},
\end{aligned}
$$

while the fermionic contribution to the one-loop partition function is the same as in (3.12).

The advantage of the static-gauge expression for the effective action is that here all fluctuation modes are decoupled and are described by elliptic differential operators of the same type, $-\partial_{\sigma}^{2}+V(\sigma)$. On general grounds, one may expect to find the same expression for the on-shell one-loop partition function in the two gauges. ${ }^{11}$ In this case one should get the following relation between the determinants of the conformal-gauge operator $\mathcal{O}_{t \rho \phi}$ in (3.7) and the static-gauge operator $\mathcal{O}_{\phi}(3.16)$ :

$$
\operatorname{det} \mathcal{O}_{t \rho \phi}=\operatorname{det} \mathcal{O}_{\phi}\left(\operatorname{det} \mathcal{O}_{0}\right)^{2}
$$

where $\mathcal{O}_{0}$ is the massless operator in (3.9). ${ }^{12} \mathrm{~A}$ concern about this equality was raised in [3] based on the fact that while the conformal-gauge one-loop partition function is UV finite [21], the static gauge one apparently contains an extra divergent term. Indeed, observing that the one-loop logarithmic UV divergence of $\ln Z$ is given by the sum of mass-squared terms and that $\bar{\mu}_{\phi}^{2}$ in (3.14) may be written in terms of the curvature of the induced metric (2.8) as $\bar{\mu}_{\phi}^{2}=\sqrt{-g}\left(4+R^{(2)}\right)$, one finds that this extra divergence is proportional to $\int \mathrm{d} \tau \mathrm{d} \sigma \sqrt{-g} R^{(2)}$. This is proportional to the Euler number of the world surface, so one may suggest [3] that it may be cancelled by the contribution of some extra 'topological' factor representing the ratio of measures in the Polyakov and Nambu path integrals.

However, this extra divergence actually vanishes in the case of a cylindrical world sheet appropriate for computing the correction to the energy of a closed string state: since $\sqrt{-g} R^{(2)}$ is a total divergence, as long as the induced metric and thus its curvature is defined to be periodic in $\sigma$, the integral over $\sigma$ should vanish. ${ }^{13}$

As we shall explicitly show in section 5 below, the effective action in the static gauge given by the sum of (3.15) and (3.11) is indeed UV finite. Moreover, we shall also verify relation (3.17), i.e. demonstrate the equivalence of the conformal-gauge and static-gauge results for the finite one-loop correction to the folded string energy. In [3] this equivalence was seen only in the long-string (infinite-spin) limit when solution (2.2) approaches the following asymptotic solution [19] $(\omega \rightarrow \kappa \gg 1)$ :

$$
t=\kappa \tau, \quad \phi=\kappa \tau, \quad \rho=\kappa \sigma, \quad \kappa=\frac{1}{\pi} \ln \mathcal{S} \gg 1,
$$

for which $\rho^{\prime}=\kappa=$ const, $R^{(2)}=0$ and relation (3.17) can be easily checked.

${ }^{11}$ For example, in the case of string theory in flat target space the static-gauge Nambu and conformal-gauge Polyakov one-loop partition functions (with non-trivial boundary conditions on a disc) are indeed the same [20].

${ }^{12}$ Here we assume that one of the massless decoupled modes is time-like, like time mode in $\mathcal{O}_{t \rho \phi}$.

${ }^{13}$ More explicitly, here the relevant integral is $\int_{0}^{2 \pi} \mathrm{d} \sigma\left(\ln \rho^{\prime}\right)^{\prime \prime}=\left[\left(\ln \rho^{\prime}\right)^{\prime}\right]_{0}^{2 \pi}=\left[\frac{\rho^{\prime \prime}}{\rho^{\prime}}\right]_{0}^{\pi / 2}+\left[\frac{\rho^{\prime \prime}}{\rho^{\prime}}\right]_{\pi / 2}^{\pi}+\left[\frac{\rho^{\prime \prime}}{\rho^{\prime}}\right]_{\pi}^{3 / 2 \pi}+\left[\frac{\rho^{\prime \prime}}{\rho^{\prime}}\right]_{3 / 2 \pi}^{2 \pi}$, where $\rho^{\prime \prime}=\left(\kappa^{2}-\omega^{2}\right) \sinh \rho \cosh \rho, \rho^{\prime}= \pm \sqrt{\kappa^{2} \cosh ^{2} \rho-\omega^{2} \sinh ^{2} \rho}$. While there is an apparent singularity at the turning points were $\rho^{\prime}=0$, this integral should vanish. One may consider using a suitable regularization of the turning points to make this vanishing manifest. 
Proving (3.17) analytically for any $\kappa$ by direct approach appears to be non-trivial. One indirect way to demonstrate (3.17) is to note that since the corresponding quadratic fluctuation operators appear in the linearized (near folded string solution) form of the string equations of motion in the two gauges, one may be able to relate these operators by relating the two sets of equations.

The conformal-gauge equations for small fluctuations following from (3.5)

$$
\begin{aligned}
& \left(\partial_{\tau}^{2}-\partial_{\sigma}^{2}\right) \tilde{t}+\mu_{t}^{2} \tilde{t}+2 \kappa \sinh \rho \partial_{\tau} \tilde{\rho}=0 \\
& \left(\partial_{\tau}^{2}-\partial_{\sigma}^{2}\right) \tilde{\rho}+\mu_{\rho}^{2} \tilde{\rho}+2\left(\kappa \sinh \rho \partial_{\tau} \tilde{t}-\omega \cosh \rho \partial_{\tau} \tilde{\phi}\right)=0 \\
& \left(\partial_{\tau}^{2}-\partial_{\sigma}^{2}\right) \tilde{\phi}+\mu_{\phi}^{2} \tilde{\phi}+2 \omega \cosh \rho \partial_{\tau} \tilde{\rho}=0,
\end{aligned}
$$

should be supplemented with the conformal-gauge conditions (Virasoro constraints)

$-\kappa \cosh ^{2} \rho \partial_{\tau} \tilde{t}+\left(\omega^{2}-\kappa^{2}\right) \sinh \rho \cosh \rho \tilde{\rho}+\rho^{\prime} \partial_{\sigma} \tilde{\rho}+\omega \sinh ^{2} \rho \partial_{\tau} \tilde{\phi}=0$

$-\kappa \cosh ^{2} \rho \partial_{\sigma} \tilde{t}+\omega \sinh ^{2} \rho \partial_{\sigma} \tilde{\phi}+\rho^{\prime} \partial_{\tau} \tilde{\rho}=0$.

The latter should allow one, in principle, to eliminate the two modes (say $\tilde{t}$ and $\tilde{\phi}$ ) in terms of the third one $(\tilde{\rho})$, getting an effective equation for the latter. Since the $\rho$-background does not depend on $\tau$ and since the above equations are linear, we may do this elimination at the Fourier mode level, i.e. replacing $\tilde{t} \rightarrow \mathrm{e}^{\mathrm{i} \Omega \tau} \bar{t}(\sigma), \tilde{\phi} \rightarrow \mathrm{e}^{\mathrm{i} \Omega \tau} \bar{\phi}(\sigma), \tilde{\rho} \rightarrow \mathrm{e}^{\mathrm{i} \Omega \tau} \bar{\rho}(\sigma)$. Then (3.22) and (3.23) imply (changing to the Euclidean time notation, i.e. $\Omega \rightarrow$ i $\Omega$ )

$$
\begin{aligned}
& \bar{t}=\frac{\sinh \rho}{2 \kappa \Omega}\left(\partial_{\sigma}^{2}-2 \rho^{\prime} \partial_{\sigma}+\kappa^{2}-\omega^{2}-\Omega^{2}\right) \bar{\rho}, \\
& \bar{\phi}=-\frac{\cosh \rho}{2 \omega \Omega}\left(\partial_{\sigma}^{2}+2 \rho^{\prime} \partial_{\sigma}-\kappa^{2}+\omega^{2}-\Omega^{2}\right) \bar{\rho} .
\end{aligned}
$$

Substituting this into the equations of motion (3.19)-(3.21) we find that one of them is satisfied automatically while the other two become equivalent to the following fourth-order differential equation for $\tilde{\rho}$, i.e. $\mathcal{O}^{(4)} \bar{\rho}=0$, where

$\mathcal{O}^{(4)} \equiv \partial_{\sigma}^{4}+2\left(\omega^{2}+\kappa^{2}-\Omega^{2}-4 \rho^{\prime 2}\right) \partial_{\sigma}^{2}-8 \rho^{\prime} \rho^{\prime \prime} \partial_{\sigma}+\kappa^{4}+\left(\Omega^{2}+\omega^{2}\right)^{2}+2 \kappa^{2}\left(\Omega^{2}-\omega^{2}\right)$.

Remarkably, this operator can be factorized as a product of two second-order operators as follows:

$$
\mathcal{O}^{(4)}=\mathcal{O}_{1} \cdot \mathcal{O}_{2}, \quad \mathcal{O}_{1}=\left(\rho^{\prime}\right)^{-1} \mathcal{O}_{\phi} \rho^{\prime}, \quad \mathcal{O}_{2}=\rho^{\prime} \mathcal{O}_{0}\left(\rho^{\prime}\right)^{-1},
$$

where $\mathcal{O}_{\phi}$ and $\mathcal{O}_{0}$ are the same as the static-gauge operator in (3.16) and the massless mode operator in (3.9), respectively. The algebraic $\rho^{\prime}$ and $\left(\rho^{\prime}\right)^{-1}$ factors may be attributed to a change of normalization of the corresponding fluctuations. ${ }^{14}$ This way we see how the staticgauge operator $\mathcal{O}_{\phi}$ emerges from the mixed conformal-gauge fluctuation operator, i.e. provides support for relation (3.17).

In section 5 below we shall verify that the effective action in the static gauge is indeed UV finite, and we demonstrate the equivalence (3.17) between the conformal-gauge and the static-gauge results for the finite one-loop correction to the folded string energy by computing the corresponding functional determinants.

\footnotetext{
${ }^{14}$ A similar discussion could be given at the level of path integral with the conformal-gauge condition accounted for by two delta-functions (as appropriate if one starts with the Nambu path integral). The step analogous to (3.24) and (3.25) would then produce an extra $\operatorname{det} \mathcal{O}_{0}$ factor as required for balance of degrees of freedom. For a discussion of the equivalence of conformal-gauge and static-gauge partition functions in a simpler case of a homogeneous string solution, see also [23].
} 


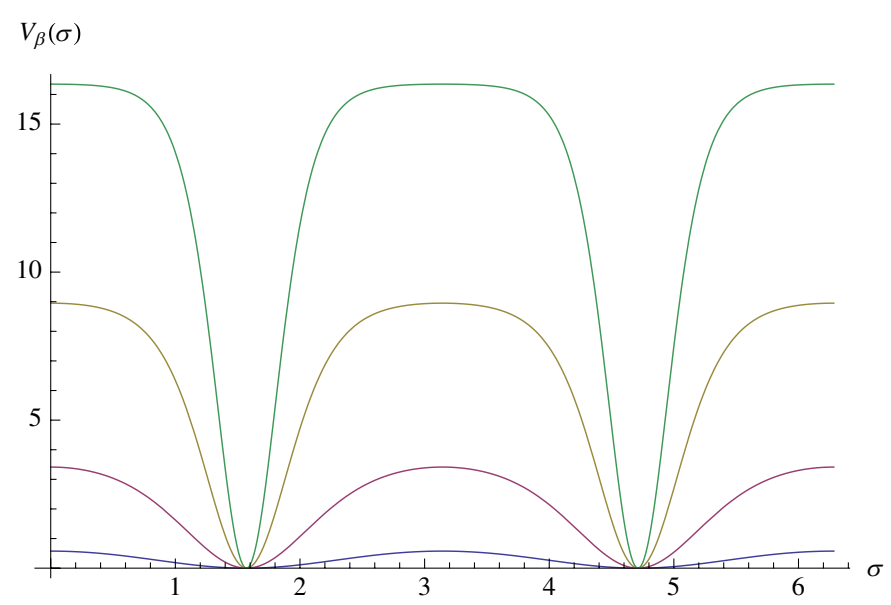

Figure 2. Potential $V_{\beta}$ in (3.29), for $k=0.5,0.9,0.99$ and 0.999 , from bottom to top.

\subsection{Lamé form of the second-order fluctuation operators}

To summarize, the simplest starting point for computing the one-loop correction to the folded string energy is thus its representation in terms of the one-loop effective action in the static gauge given by the sum of (3.15) and (3.11), i.e. is expressed in terms of the determinants of the following three types of operators defined on periodic functions $f=\left(\beta, \phi, \psi_{ \pm}\right)$:

$$
\mathcal{O}_{f}=-\partial_{\sigma}^{2}+V_{f}(\sigma)+\Omega^{2}, \quad f(\sigma)=f(\sigma+2 \pi),
$$

where (using the form of the classical solution (2.4))

$$
\begin{aligned}
& V_{\beta}=2 \rho^{\prime 2}=2 \kappa^{2} \operatorname{sn}^{2}\left(\bar{\sigma} \mid k^{2}\right), \quad \bar{\sigma} \equiv \omega \sigma+\mathbb{K} \\
& V_{\phi}=2 \rho^{\prime 2}+\frac{2 \kappa^{2} \omega^{2}}{\rho^{\prime 2}}=2 \kappa^{2} \operatorname{sn}^{2}\left(\bar{\sigma} \mid k^{2}\right)+2 \omega^{2} \mathrm{~ns}^{2}\left(\bar{\sigma} \mid k^{2}\right) \\
& V_{\psi_{ \pm}}=\rho^{\prime 2} \pm \rho^{\prime \prime}=\kappa^{2} \operatorname{sn}^{2}\left(\bar{\sigma} \mid k^{2}\right) \pm \kappa \omega \operatorname{cn}\left(\bar{\sigma} \mid k^{2}\right) \operatorname{dn}\left(\bar{\sigma} \mid k^{2}\right)
\end{aligned}
$$

These potentials are plotted in figures 2, 3 and 4 where we have chosen four particular values of the elliptic modulus $k=\frac{\kappa}{\omega}=0.5,0.9,0.99,0.999$.

It is convenient to introduce the rescaled spatial variable (cf (2.7))

$$
x \equiv \omega \sigma=\frac{2 \mathbb{K}}{\pi} \sigma
$$

and write $\mathcal{O}_{\beta}$ as (we ignore a trivial overall constant factor)

$$
\mathcal{O}_{\beta}=-\partial_{x}^{2}+2 k^{2} \operatorname{sn}^{2}\left(x+\mathbb{K} \mid k^{2}\right)+\frac{\pi^{2} \Omega^{2}}{4 \mathbb{K}^{2}},
$$

which is now defined on the periodic functions $\beta(x)=\beta(x+4 \mathbb{K})$. The expression in (3.33) is recognized as being a Lamé differential operator in the single-gap form (which will be reviewed in the next section).

Remarkably, all other fluctuation operators entering the effective action, i.e. $\mathcal{O}_{\phi}$ and $\mathcal{O}_{\psi_{ \pm}}$, whose structure is apparently much more involved, can also be cast into the single-gap Lamé form. Their transformation has several steps involving rescaling the coordinate and the elliptic modulus in a special way, see (A.18)-(A.21) for $\mathcal{O}_{\phi}$, and (A.22)-(A.31) for $\mathcal{O}_{\psi_{ \pm}}$. 


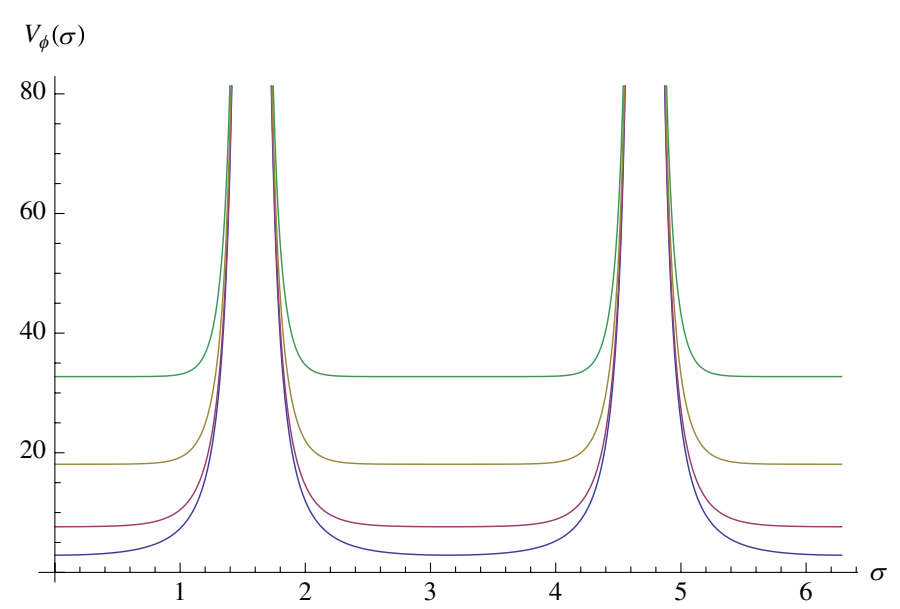

Figure 3. Potential $V_{\phi}$ in (3.30), for $k=0.5,0.9,0.99$ and 0.999 , from bottom to top. Note that singularities appear at the turning points where $\sigma=\left(n+\frac{1}{2}\right) \pi$.

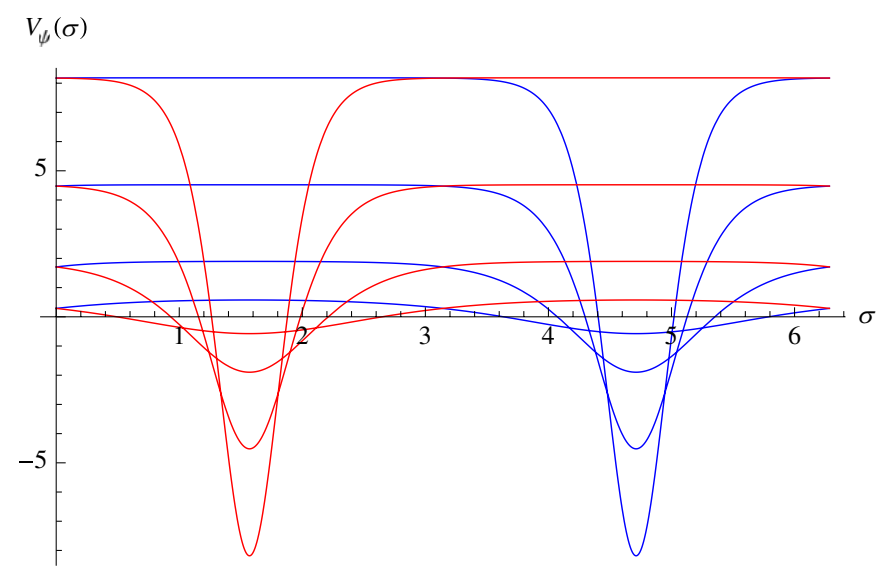

Figure 4. Potentials $V_{\psi_{+}}$(red) and $V_{\psi_{-}}$(blue) in (3.31), for $k=0.5,0.9,0.99$ and 0.999 , from bottom to top. Note that $V_{ \pm}(\sigma)$ are identical in from, but are displaced from one another by a half-period $\pi$; they are self-isospectral [26].

We can summarize the results as follows: each (static-gauge) fluctuation operator is a single-gap Lamé operator with the following periodic eigenvalue problem:

$\left[-\partial_{x}^{2}+2 \bar{k}^{2} \operatorname{sn}^{2}\left(x \mid \bar{k}^{2}\right)+\bar{\Omega}^{2}\right] f_{\Lambda}(x)=\Lambda f_{\Lambda}(x), \quad f_{\Lambda}(x)=f_{\Lambda}(x+L)$,

where $x$ is a rescaled $\sigma$ variable with period $L$, and $\bar{k}$ and $\bar{\Omega}$ are the rescaled modulus and Euclidean frequency in (3.29)-(3.31), namely,

(a) for the bosonic operator $\mathcal{O}_{\beta}$ :

$$
x=\frac{2 \mathbb{K}}{\pi} \sigma+\mathbb{K}, \quad \bar{k}=k, \quad \bar{\Omega}^{2}=\left(\frac{\pi \Omega}{2 \mathbb{K}}\right)^{2}, \quad L=4 \mathbb{K}
$$


(b) for the bosonic operator $\mathcal{O}_{\phi}$ : the elliptic modulus is $\tilde{k}^{2}=\frac{4 k}{(1+k)^{2}}$ and

$$
x=\frac{2 \widetilde{\mathbb{K}}}{\pi} \sigma+\mathrm{i} \tilde{\mathbb{K}}^{\prime}, \quad \bar{k}=\tilde{k} \equiv \frac{2 \sqrt{k}}{1+k}, \quad \bar{\Omega}^{2}=\left(\frac{\pi \Omega}{2 \widetilde{\mathbb{K}}}\right)^{2}+\tilde{k}^{2}, \quad L=4 \widetilde{\mathbb{K}}
$$

(c) for the fermionic operators $\mathcal{O}_{\psi_{ \pm}}$:

$$
\begin{aligned}
& x= \begin{cases}\frac{\widetilde{\mathbb{K}}}{\pi} \sigma+\frac{\widetilde{\mathbb{K}}}{2}, & \text { for } \quad \psi_{+}, \\
\frac{\widetilde{\mathbb{K}}}{\pi} \sigma+\frac{3 \widetilde{\mathbb{K}}}{2}, & \text { for } \quad \psi_{-}\end{cases} \\
& \bar{k}=\tilde{k} \equiv \frac{2 \sqrt{k}}{1+k}, \quad \bar{\Omega}^{2}=\left(\frac{\pi \Omega}{\widetilde{\mathbb{K}}}\right)^{2}+\tilde{k}^{2}, \quad L=2 \widetilde{\mathbb{K}} .
\end{aligned}
$$

Here $\widetilde{\mathbb{K}} \equiv \mathbb{K}\left(\tilde{k}^{2}\right)$, and $k^{\prime} \equiv \sqrt{1-k^{2}}$, see appendix A for notation and details.

As we shall discuss in the next section, the remarkable feature of this Lamé spectral problem (3.34) is that it can be solved exactly, and hence the corresponding determinant can be computed analytically (with a result that is independent of constant shifts in the coordinates).

\section{Determinants of single-gap Lamé operators}

Below we shall first review the method that allows one to compute the determinant of a singlegap Lamé operator without having to solve the corresponding spectral problem explicitly. We will then apply this technique to the computation of determinants of the fluctuation operators discussed in the previous section.

\subsection{Floquet theory of determinants of second-order one-dimensional operators}

Consider the following eigenvalue problem for an ordinary differential operator, $\mathcal{O}=$ $-\partial_{x}^{2}+V(x)$, with a periodic potential:

$$
\left[-\partial_{x}^{2}+V(x)\right] f(x)=\Lambda f(x), \quad V(x+L)=V(x) .
$$

For either periodic or antiperiodic boundary conditions on $f(x)$, we find a discrete spectrum of eigenvalues $\left\{\Lambda_{n}\right\}$, and the associated determinant is then formally given by $\operatorname{det} \mathcal{O}=\prod_{n} \Lambda_{n}$.

Given a general potential $V(x)$, it is of course difficult to find the eigenvalues, and even given the eigenvalues, the infinite product must be regulated. Both difficulties can be overcome in the following way. Consider two independent solutions $f_{1,2}(x ; \Lambda)$ to $(4.1)$ satisfying the conditions

$$
\begin{array}{ll}
f_{1}(0 ; \Lambda)=1, & f_{1}^{\prime}(0 ; \Lambda)=0, \\
f_{2}(0 ; \Lambda)=0, & f_{2}^{\prime}(0 ; \Lambda)=1,
\end{array}
$$

where $f^{\prime}=\partial_{x} f$. Then the discriminant $\Delta(\Lambda)$ of the operator $\mathcal{O}$ is defined as [31]

$$
\Delta(\Lambda)=f_{1}(L ; \Lambda)+f_{2}^{\prime}(L ; \Lambda)
$$

The periodic and the antiperiodic eigenvalues are given by the following (in general transcendental) equations:

$$
\Delta(\Lambda)= \begin{cases}+2 & \text { (periodic) } \\ -2 & \text { (antiperiodic) }\end{cases}
$$

Remarkably, the determinant can be computed without knowing these eigenvalues explicitly. Indeed, the Hill determinant, i.e. the ratio of determinants with non-zero $V$ and $V=0$, has a simple expression in terms of the discriminant [31]: 


$$
\begin{aligned}
& \frac{\operatorname{det}\left[-\partial_{x}^{2}+V(x)-\Lambda\right]}{\operatorname{det}\left[-\partial_{x}^{2}-\Lambda\right]}=\frac{\Delta(\Lambda)-2}{-4 \sin ^{2}(L \sqrt{\Lambda} / 2)} \quad \text { (periodic) } \\
& \frac{\operatorname{det}\left[-\partial_{x}^{2}+V(x)-\Lambda\right]}{\operatorname{det}\left[-\partial_{x}^{2}-\Lambda\right]}=\frac{\Delta(\Lambda)+2}{4 \cos ^{2}(L \sqrt{\Lambda} / 2)} \quad \text { (antiperiodic) }
\end{aligned}
$$

In what follows we shall always assume that determinants we consider are normalized to the trivial free determinant $\operatorname{det}\left[-\partial_{x}^{2}\right]$, and thus omit the resulting $\Lambda$ - and $V$-independent overall constant (such constants will cancel in the string partition function due to the balance of the degrees of freedom). Then we may write the above relations simply as

$$
\operatorname{det}_{P, A P}\left[-\partial_{x}^{2}+V(x)-\Lambda\right]= \begin{cases}\Delta(\Lambda)-2 & \text { (periodic) } \\ \Delta(\Lambda)+2 & \text { (antiperiodic) }\end{cases}
$$

It is useful to relate this representation for the determinant to a familiar physical notion of 'quasi-momentum'. By the Floquet-Bloch theory [31], equation (4.1) has two independent solutions of the form $f_{ \pm}(x)=\mathrm{e}^{ \pm \mathrm{i} p(\Lambda) x} \chi_{ \pm}(x)$, where $\chi_{ \pm}(x)$ are periodic, so that under translation through one period the Bloch solutions $f_{ \pm}(x)$ change by a phase

$$
f_{ \pm}(x+L)=\mathrm{e}^{ \pm \mathrm{i} p(\Lambda) L} f_{ \pm}(x)
$$

where, by definition, $p(\Lambda)$ is the 'quasi-momentum'. Then $\Delta(\Lambda)=2 \cos (L p(\Lambda))$, and we can rewrite (4.7) in terms of the quasi-momentum as follows [31]:

$\operatorname{det}_{P, A P}\left[-\partial_{x}^{2}+V(x)-\Lambda\right]= \begin{cases}-4 \sin ^{2}\left(\frac{L}{2} p(\Lambda)\right) & \text { (periodic) } \\ +4 \cos ^{2}\left(\frac{L}{2} p(\Lambda)\right) & \text { (antiperiodic) }\end{cases}$

Thus, knowing the quasi-momentum $p(\Lambda)$ amounts to knowing the discriminant and also the determinant.

Another interesting and useful relation is the link between the determinant and the discriminant through the contour integral representation for the spectral zeta function. For definiteness, let us consider the case of the periodic boundary conditions. Then the spectral zeta function is

$$
\zeta(s)=\frac{1}{2 \pi \mathrm{i}} \int_{\gamma} \mathrm{d} \Lambda \Lambda^{-s} \frac{\partial}{\partial \Lambda} \ln [\Delta(\Lambda)-2]=\frac{1}{2 \pi \mathrm{i}} \int_{\gamma} \mathrm{d} \Lambda \Lambda^{-s} R(\Lambda),
$$

where the resolvent

$$
R(\Lambda)=\frac{\Delta^{\prime}(\Lambda)}{\Delta(\Lambda)-2}
$$

has simple poles exactly at the values of $\Lambda$ corresponding to the points of the periodic spectrum. The contour $\gamma$ in (4.10) runs counterclockwise above and below the positive real axis enclosing all poles of the resolvent. Wrapping the contour along the branch cut along the negative real line gives [25]

$$
\zeta(s)=-\frac{\sin (\pi s)}{\pi} \int_{0}^{\infty} \mathrm{d} \Lambda \Lambda^{-s} R(-\Lambda) .
$$

According to the zeta function definition of the functional determinant

$$
\operatorname{det}\left[-\partial_{x}^{2}+V(x)\right]=\mathrm{e}^{-\zeta^{\prime}(0)}
$$

to compute the determinant we need to know

$$
-\zeta^{\prime}(0)=-\int_{0}^{\infty} \mathrm{d} \Lambda \frac{\partial}{\partial \Lambda} \ln [\Delta(-\Lambda)-2]=\ln \frac{[\Delta(0)-2]}{[\Delta(-\infty)-2]} .
$$


Here we subtracted the divergent term $\ln [\Delta(-\infty)-2]$ by assuming that we again divide by a 'free' reference determinant. Then finally

$$
\operatorname{det}_{P}\left[-\partial_{x}^{2}+V(x)\right]=\Delta(0)-2 .
$$

Shifting the potential by a constant $-\Lambda$, we reproduce the representation in (4.7).

The important feature of the above expressions is that the determinants can be calculated in closed form without computing any of the eigenvalues. There is yet another way to compute the determinants, known as the Gel'fand-Yaglom method [28, 35], which for periodic systems reduces essentially to a numerical evaluation of the discriminant giving the determinants via (4.7). This method is described in appendix B, where we also consider systems of coupled equations, which will be important for demonstrating explicitly the equivalence of the computation in the conformal and static gauges.

It is useful to illustrate the above general relations on the simple example of the constant potential

$$
V(x)=m^{2}, \quad x \in(0, L) .
$$

Then the two independent solutions in (4.2) are $f_{1}(x ; \Lambda)=\cosh \left(\sqrt{m^{2}-\Lambda} x\right)$ and $f_{2}(x ; \Lambda)=$ $\sinh \left(\sqrt{m^{2}-\Lambda} x\right) / \sqrt{m^{2}-\Lambda}$. Therefore, discriminant (4.3) and determinants (4.9) are

$$
\begin{aligned}
& \Delta(\Lambda)=2 \cosh \left(L \sqrt{m^{2}-\Lambda}\right) \\
& \operatorname{det}_{P, A P}\left(-\partial_{x}^{2}+m^{2}-\Lambda\right)=\left\{\begin{array}{l}
4 \sinh ^{2}\left(\frac{L}{2} \sqrt{m^{2}-\Lambda}\right) \\
4 \cosh ^{2}\left(\frac{L}{2} \sqrt{m^{2}-\Lambda}\right) .
\end{array}\right.
\end{aligned}
$$

The quasi-momentum in (4.8) here is $p(\Lambda)=\sqrt{\Lambda-m^{2}}$, so these relations are consistent with (4.9). Furthermore, in this case we know the explicit eigenvalues $(n \in \mathbb{Z})$ :

$$
\Lambda_{n}= \begin{cases}m^{2}+\left(\frac{2 n \pi}{L}\right)^{2} & \text { (periodic) } \\ m^{2}+\left(\frac{(2 n+1) \pi}{L}\right)^{2} & \text { (antiperiodic) }\end{cases}
$$

Then expressions (4.18) also follow from the infinite product representations for the sinh and cosh functions, combined with zeta function regularization.

\subsection{Case of the single-gap Lamé potential $V(x)=2 k^{2} \operatorname{sn}^{2}\left(x \mid k^{2}\right)$}

The important example which is our main interest here is provided by the single-gap Lamé operator in (3.34), i.e.

$$
\left[-\partial_{x}^{2}+2 k^{2} \operatorname{sn}^{2}\left(x \mid k^{2}\right)\right] f(x)=\Lambda f(x)
$$

The two independent Bloch solutions of (4.20) here are [30]

$$
f_{ \pm}(x)=\frac{H(x \pm \alpha)}{\Theta(x)} \mathrm{e}^{\mp x Z(\alpha)}
$$

where $H, \Theta, Z$ are the Jacobi eta, theta and zeta functions defined in (A.10), and $\alpha=\alpha(\Lambda)$ is given implicitly by

$$
\operatorname{sn}\left(\alpha \mid k^{2}\right)=\sqrt{\frac{1+k^{2}-\Lambda}{k^{2}}} .
$$




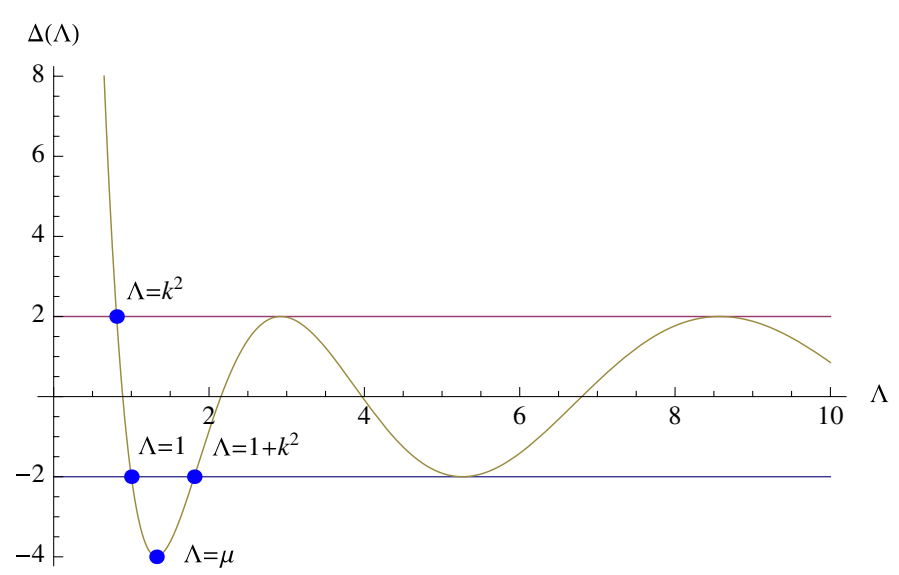

Figure 5. The discriminant $\Delta(\Lambda)$ for the Lamé potential $V(x)=2 k^{2} \operatorname{sn}^{2}\left(x \mid k^{2}\right)$ with $k=0.9$. The three band edges occur at the points $\Lambda_{1}, \Lambda_{2}, \Lambda_{3}$ where $\Delta(\Lambda)$ cuts the lines \pm 2 , while the remainder of the periodic/antiperiodic spectrum consists of points where $\Delta(\Lambda)$ touches the lines \pm 2 .

Using the period properties of the Jacobi functions (A.12) we see that

$$
f_{ \pm}(x+2 \mathbb{K})=-f_{ \pm}(x) \mathrm{e}^{\mp 2 \mathbb{K} Z(\alpha)} \equiv f_{ \pm}(x) \mathrm{e}^{2 \mathrm{i} \mathbb{K} p(\alpha)},
$$

which defines the quasi-momentum as

$$
p(\Lambda)=\mathrm{i} Z\left(\alpha \mid k^{2}\right)+\frac{\pi}{2 \mathbb{K}} .
$$

Therefore, from (4.9) we immediately find analytic expressions for the determinants. Assuming the period is $L=2 \mathbb{K}$, we find

$$
\operatorname{det}_{P, A P}^{(L=2 \mathbb{K})}\left[-\partial_{x}^{2}+2 k^{2} \operatorname{sn}^{2}\left(x \mid k^{2}\right)-\Lambda\right]=\left\{\begin{array}{l}
-4 \cosh ^{2}\left[\mathbb{K} Z\left(\alpha \mid k^{2}\right)\right] \\
-4 \sinh ^{2}\left[\mathbb{K} Z\left(\alpha \mid k^{2}\right)\right]
\end{array}\right.
$$

where the relation between $\Lambda$ and $\alpha$ is given by (4.22). On the other hand, for the period $L=4 \mathbb{K}$, we find

$$
\operatorname{det}_{P}^{(L=4 \mathbb{K})}\left[-\partial_{x}^{2}+2 k^{2} \operatorname{sn}^{2}\left(x \mid k^{2}\right)-\Lambda\right]=4 \sinh ^{2}\left[2 \mathbb{K} Z\left(\alpha \mid k^{2}\right)\right] .
$$

Note that this is the same as the product of the periodic and antiperiodic determinants (4.25) with the period $2 \mathbb{K}$, as it should be.

The periodic potential in (4.20) has a special property that its band spectrum has only a single gap, and is known therefore as a one-gap potential, as illustrated in figure 5. The spectrum has three band edges (which are also the lowest eigenvalues of the periodic spectrum of the problem on the interval $4 \mathbb{K})$ :

$$
\Lambda_{1}=k^{2}, \quad \Lambda_{2}=1, \quad \Lambda_{3}=1+k^{2} .
$$

One can rewrite the relation between $\Lambda$ and $\alpha$ in (4.22) in terms of the band edges as follows:

$$
k \operatorname{sn}\left(\alpha ; k^{2}\right)=\sqrt{\frac{1}{2}\left(\Lambda_{1}+\Lambda_{2}+\Lambda_{3}\right)-\Lambda} .
$$

The resolvent is

$$
R(\Lambda)=\frac{\mathrm{d}}{\mathrm{d} \Lambda} \ln [\Delta(\Lambda)-2]=L \frac{\mathrm{d} p}{\mathrm{~d} \Lambda} \cot \left[\frac{L}{2} p(\Lambda)\right]
$$


We can also express $\mathrm{d} p / \mathrm{d} \Lambda$ simply in terms of the band edges:

$$
\frac{\mathrm{d} p}{\mathrm{~d} \Lambda}=\mathrm{i} \frac{\Lambda-\mu}{2 \sqrt{\left(\Lambda_{1}-\Lambda\right)\left(\Lambda_{2}-\Lambda\right)\left(\Lambda_{3}-\Lambda\right)}}
$$

where

$$
\mu=\frac{1}{2}\left(\Lambda_{1}+\Lambda_{2}+\Lambda_{3}-\langle V\rangle\right), \quad\langle V\rangle \equiv \frac{1}{L} \int_{0}^{L} V(x) \mathrm{d} x .
$$

To see this, note that from (4.8)

$$
\frac{\mathrm{d} p}{\mathrm{~d} \Lambda}=\frac{\mathrm{d} p}{\mathrm{~d} \alpha} \frac{\mathrm{d} \alpha}{\mathrm{d} \Lambda}=\mathrm{i} \frac{\mathrm{d} Z\left(\alpha \mid k^{2}\right)}{\mathrm{d} \alpha} \frac{\mathrm{d} \alpha}{\mathrm{d} \Lambda}=\mathrm{i}\left(1-k^{2} \operatorname{sn}^{2}\left(\alpha \mid k^{2}\right)-\frac{\mathbb{E}\left(k^{2}\right)}{\mathbb{K}\left(k^{2}\right)}\right) \frac{\mathrm{d} \alpha}{\mathrm{d} \Lambda}
$$

where we have used the definition (A.16) of the zeta function. Also, from (4.28) we have $\frac{\mathrm{d} \alpha}{\mathrm{d} \Lambda}=\frac{1}{2 k^{2} \operatorname{dn}\left(\alpha \mid k^{2}\right) \operatorname{cn}\left(\alpha \mid k^{2}\right) \operatorname{sn}\left(\alpha \mid k^{2}\right)}=\frac{1}{2 \sqrt{\left(\Lambda_{1}-\Lambda\right)\left(\Lambda_{2}-\Lambda\right)\left(\Lambda_{3}-\Lambda\right)}}$.

Finally, for the potential $V(x)=2 k^{2} \operatorname{sn}^{2}\left(x \mid k^{2}\right)$ we find for $L=2 \mathbb{K}$

$$
\langle V\rangle=\frac{1}{L} \int_{0}^{L} \mathrm{~d} x 2 k^{2} \operatorname{sn}^{2}\left(x \mid k^{2}\right)=2\left(1-\frac{\mathbb{E}\left(k^{2}\right)}{\mathbb{K}\left(k^{2}\right)}\right),
$$

and the same for $L=4 \mathbb{K}$. Thus, taking into account (4.27), we get

$$
\mu=k^{2}+\frac{\mathbb{E}\left(k^{2}\right)}{\mathbb{K}\left(k^{2}\right)}
$$

Thus we obtain a compact expression for the resolvent of the single-gap Lamé potential with period $L$ in terms of the quasi-momentum $p(\Lambda)$ and the band edges as

$$
R(\Lambda)=\frac{L}{2} \frac{\Lambda-\mu}{\sqrt{\left(\Lambda_{1}-\Lambda\right)\left(\Lambda_{2}-\Lambda\right)\left(\Lambda_{3}-\Lambda\right)}} \operatorname{coth}\left(\frac{L p(\Lambda)}{2 i}\right) .
$$

\subsection{Results for determinants of static-gauge fluctuation operators}

Let us now apply the above results to the case of the fluctuation operators defined by (3.34)(3.37). The results in equations (4.22) and (4.25)-(4.26) are actually all that we need in order to write down exact analytic expressions for the determinants of these operators. The analytically known eigenvalues or band edges can be obtained from (4.27) with the appropriate shifts $\left(\Lambda_{i} \rightarrow \Lambda_{i}-\bar{\Omega}^{2}\right)$ and rescalings, and an analogous procedure applies to the corresponding resolvents in (4.34).

The results can be summarized as follows.

(a) For the $\beta$ operator, in view of (3.35), the determinant reads

$$
\begin{aligned}
& \operatorname{det} \mathcal{O}_{\beta}(\Omega)=4 \sinh ^{2}\left[2 \mathbb{K} Z\left(\alpha_{\beta} \mid k^{2}\right)\right], \\
& \operatorname{sn}\left(\alpha_{\beta} ; k^{2}\right)=\frac{\sqrt{1+k^{2}+\left(\frac{\pi \Omega}{2 \mathbb{K}}\right)^{2}}}{k}
\end{aligned}
$$

The band edges are obtained from (4.27) by shifting and rescaling

$$
\bar{\Lambda}_{i}=\left(\frac{2 \mathbb{K}}{\pi}\right)^{2}\left(\Lambda_{i}+\bar{\Omega}^{2}\right) \equiv \Omega_{i}^{2}+\Omega^{2}, \quad \bar{\Omega}^{2}=\left(\frac{\pi \Omega}{2 \mathbb{K}}\right)^{2}
$$


Table 1. The lowest (analytically known) eigenvalues of the fluctuation operators.

\begin{tabular}{llll}
\hline & $\beta$ & $\phi$ & Fermions \\
\hline $\bar{\Lambda}_{1}=\Omega_{1}^{2}+\Omega^{2}$ & $\kappa^{2}+\Omega^{2}$ & $\Omega^{2}$ & $\Omega^{2}$ \\
$\bar{\Lambda}_{2}=\Omega_{2}^{2}+\Omega^{2}$ & $\omega^{2}+\Omega^{2}$ & $(\omega-\kappa)^{2}+\Omega^{2}$ & $\frac{1}{4}(\omega-\kappa)^{2}+\Omega^{2}$ \\
$\bar{\Lambda}_{3}=\Omega_{3}^{2}+\Omega^{2}$ & $\omega^{2}+\kappa^{2}+\Omega^{2}$ & $(\omega+\kappa)^{2}+\Omega^{2}$ & $\frac{1}{4}(\omega+\kappa)^{2}+\Omega^{2}$ \\
$\bar{\mu}$ & $\kappa^{2}+\omega^{2} \frac{\mathbb{E}}{\mathbb{K}}$ & $-\left(\omega^{2}-\kappa^{2}\right)+2 \omega^{2} \frac{\mathbb{E}}{\mathbb{K}}$ & $-\frac{1}{4}\left(\omega^{2}-\kappa^{2}\right)+\frac{\omega^{2}}{2} \frac{\mathbb{E}}{\mathbb{K}}$ \\
\hline
\end{tabular}

where the rescaled $\Lambda_{i}$ have been defined as 'characteristic frequencies' $\Omega_{i}^{2}$. One thus gets the eigenvalues in the first column of table 1 that can now be re-expressed in terms of the parameters of the classical solution:

$$
\begin{aligned}
\left\{\bar{\Lambda}_{1}, \bar{\Lambda}_{2}, \bar{\Lambda}_{3}\right\} & =\left(\frac{2 \mathbb{K}}{\pi}\right)^{2}\left\{k^{2}+\bar{\Omega}^{2}, 1+\bar{\Omega}^{2}, 1+k^{2}+\bar{\Omega}^{2}\right\} \\
& \equiv\left\{\kappa^{2}+\Omega^{2}, \omega^{2}+\Omega^{2}, \kappa^{2}+\omega^{2}+\Omega^{2}\right\}
\end{aligned}
$$

(b) For the $\phi$ operator in (3.36) we have $\tilde{k}^{2}=\frac{4 k}{(k+1)^{2}}$, and thus

$$
\begin{aligned}
& \operatorname{det} \mathcal{O}_{\phi}(\Omega)=4 \sinh ^{2}\left[2 \widetilde{\mathbb{K}} Z\left(\alpha_{\phi} \mid \tilde{k}^{2}\right)\right], \\
& \operatorname{sn}\left(\alpha_{\phi} \mid \tilde{k}^{2}\right)=\frac{\sqrt{1+\left(\frac{\pi \Omega}{2 \mathbb{K}}\right)^{2}}}{\tilde{k}}
\end{aligned}
$$

The band edges are obtained from (4.27) by shifting and rescaling

$$
\bar{\Lambda}_{i}=\left(\frac{2 \widetilde{\mathbb{K}}}{\pi}\right)^{2}\left(\Lambda_{i}+\bar{\Omega}^{2}\right) \equiv \Omega_{i}^{2}+\Omega^{2}, \quad \bar{\Omega}^{2}=\left(\frac{\pi \Omega}{2 \widetilde{\mathbb{K}}}\right)^{2}+\tilde{k}^{2}
$$

getting thus the eigenvalues in the second column of table 1.

(c) For the $\psi_{ \pm}$operators in (3.37) the elliptic parameter is $\tilde{k}^{2}=\frac{4 k}{(k+1)^{2}}$, and we get

$$
\begin{aligned}
\operatorname{det} \mathcal{O}_{\psi}(\Omega) & =-4 \cosh ^{2}\left[\tilde{\mathbb{K}} Z\left(\alpha_{\psi} \mid \tilde{k}^{2}\right)\right], \\
\operatorname{sn}\left(\alpha_{\psi} \mid \tilde{k}^{2}\right) & =\frac{\sqrt{1+\left(\frac{\pi \Omega}{\widetilde{K}}\right)^{2}}}{\tilde{k}}
\end{aligned}
$$

Since the determinant is independent of the constant shifts of coordinates like the one in (3.37) (see, e.g. appendix B) the expressions for the determinants of $\mathcal{O}_{\psi_{-}}$and $\mathcal{O}_{\psi_{+}}$are the same and therefore we will not distinguish them in what follows. The band edges follow from (4.27):

$$
\bar{\Lambda}_{i}=\left(\frac{\widetilde{\mathbb{K}}}{\pi}\right)^{2}\left(\Lambda_{i}+\bar{\Omega}^{2}\right) \equiv \Omega_{i}^{2}+\Omega^{2}, \quad \bar{\Omega}^{2}=\left(\frac{\pi \Omega}{\widetilde{\mathbb{K}}}\right)^{2}+\tilde{k}^{2}
$$




\section{Exact expression for the one-loop correction to string energy}

As follows from the above discussion (see (3.2), (3.15), (3.11), (4.35), (4.39), (4.42)) the one-loop correction to the energy of the folded spinning string may be written as

$$
E_{1}=-\frac{1}{4 \pi \kappa} \int_{-\infty}^{\infty} \mathrm{d} \Omega \ln \frac{\operatorname{det}^{8} \mathcal{O}_{\psi}}{\operatorname{det}_{\phi} \operatorname{det}^{2} \mathcal{O}_{\beta} \operatorname{det}^{5} \mathcal{O}_{0}},
$$

where $\kappa=\frac{2 k}{\pi} \mathbb{K}, \quad \mathbb{K}=\mathbb{K}\left(k^{2}\right)$ (see (2.7)) and the determinants as functions of $\Omega$ have the following explicit expressions ${ }^{15}$ :

$$
\begin{array}{lll}
\operatorname{det} \mathcal{O}_{\beta}=4 \sinh ^{2}\left[2 \mathbb{K} Z\left(\alpha_{\beta} \mid k^{2}\right)\right] & \text { where } & \operatorname{sn}\left(\alpha_{\beta} \mid k^{2}\right)=\frac{\sqrt{1+k^{2}+\left(\frac{\pi \Omega}{2 \mathbb{K}}\right)^{2}}}{k} \\
\operatorname{det} \mathcal{O}_{\phi}=4 \sinh ^{2}\left[2 \widetilde{\mathbb{K}} Z\left(\alpha_{\phi} \mid \tilde{k}^{2}\right)\right] \quad \text { where } & \operatorname{sn}\left(\alpha_{\phi} \mid \tilde{k}^{2}\right)=\frac{\sqrt{1+\left(\frac{\pi \Omega}{2 \widetilde{K}}\right)^{2}}}{\tilde{k}} \\
\operatorname{det} \mathcal{O}_{\psi}=-4 \cosh ^{2}\left[\tilde{\mathbb{K}} Z\left(\alpha_{\psi} \mid \tilde{k}^{2}\right)\right] \quad \text { where } \quad \operatorname{sn}\left(\alpha_{\psi} \mid \tilde{k}^{2}\right)=\frac{\sqrt{1+\left(\frac{\pi \Omega}{\widetilde{K}}\right)^{2}}}{\tilde{k}} \\
\operatorname{det} \mathcal{O}_{0}=4 \sinh ^{2}[\pi \Omega]
\end{array}
$$

The computation of $E_{1}$ is thus reduced to inverting the transcendental equations for $\alpha_{\beta}, \alpha_{\phi}, \alpha_{\phi}$, finding the corresponding values of the $Z$-function (A.15). The integral is then a function of $k=\frac{\kappa}{\omega}$ and $\Omega .{ }^{16}$ Doing the integral over $\Omega$ we then end up with a function of $k$ only or the spin (2.11). It is straightforward to evaluate the $\Omega$ integral numerically, as discussed below.

\subsection{UV finiteness}

Let us first check that the resulting expression for $E_{1}$ is indeed UV finite, i.e. the integral over $\Omega$ is convergent at infinity. The large $\Omega$ behaviour of the determinant factors in (5.1) can be very easily extracted from the general large $\Omega$ behaviour of the associated resolvents. Changing the variable from $\Lambda$ to $-\Omega^{2}$, we define

$$
\mathcal{R}(\Omega) \equiv-2 \Omega R\left(-\Omega^{2}\right)
$$

Then we find from (4.34) that the general structure of the expansion is

$$
\begin{aligned}
& \mathcal{R}(\Omega)=r_{0}+\frac{r_{1}}{\Omega^{2}}+\frac{r_{2}}{\Omega^{4}}+\mathcal{O}\left(\Omega^{-6}\right), \quad \Omega \rightarrow \infty \\
& r_{0}=2 \pi, \quad r_{1}=2 \pi\left[\bar{\mu}-\frac{1}{2}\left(\bar{\Lambda}_{1}+\bar{\Lambda}_{2}+\bar{\Lambda}_{3}\right)\right]=2 \pi\langle V\rangle .
\end{aligned}
$$

Therefore, the large $\Omega$ behaviour of the log determinant is

$$
\ln \operatorname{det} \mathcal{O}=r_{0} \Omega-\frac{r_{1}}{\Omega}+\mathcal{O}\left(\Omega^{-3}\right), \quad \Omega \rightarrow \infty
$$

Using the corresponding values of $\bar{\mu}$ and $\mathcal{L}_{i}$ of the three non-trivial fluctuation modes given in table 1, we find (we have also used the elliptic identities (A.28) and (A.29))

15 The determinant of the massless operator $\mathcal{O}_{0}$ is found by taking the regularized infinite product of its eigenvalues $\lambda_{n}=n^{2}+\Omega^{2}$.

${ }^{16}$ Note that in the limit when $k=0$, i.e. potentials vanish, all determinants take the same value as $\operatorname{det} \mathcal{O}_{0}$, i.e. $E_{1}$ in (5.1) vanishes. 


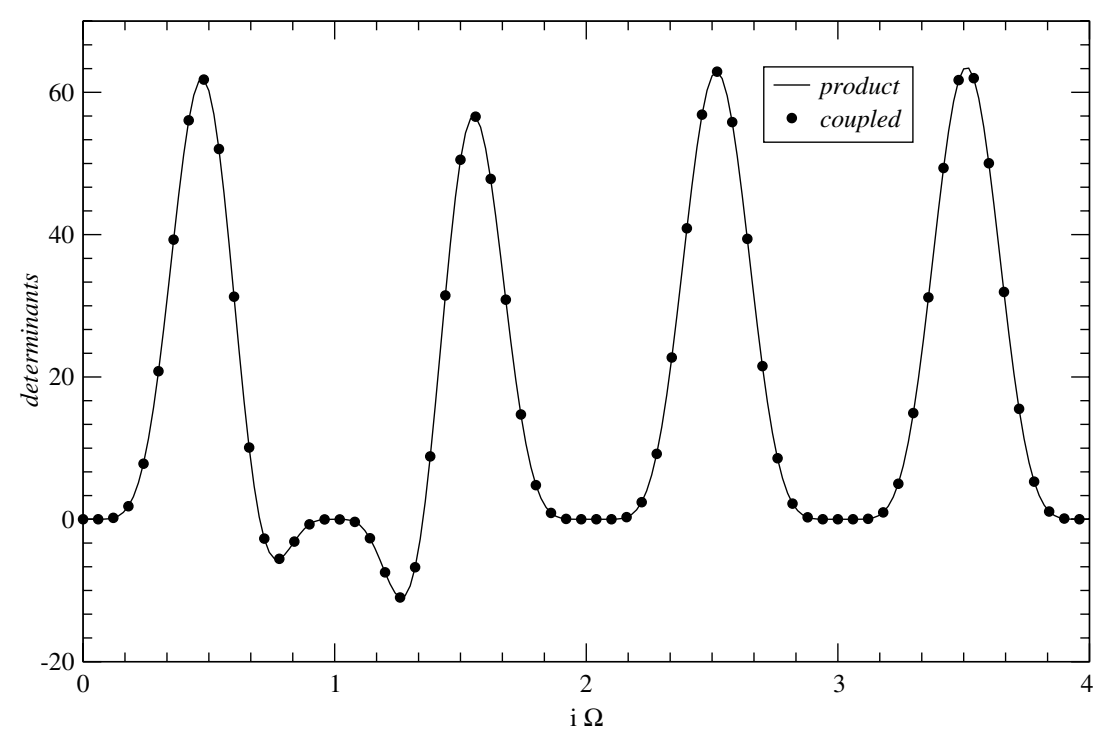

Figure 6. Comparison between the left-hand side and the right-hand side of equation (3.17) for $k=\frac{1}{\sqrt{10}}$. The circles represent the numerical Gel'fand-Yaglom result for the determinant $\operatorname{det} \mathcal{O}_{t \rho \phi}$ of the three coupled fluctuations in conformal gauge, while the solid line is a plot of the corresponding analytic static-gauge expression, given by the product of the determinant (5.3) for the massive fluctuation $\phi$ and the square of the determinant (5.5) of a massless mode. To emphasize the precision of the agreement we have plotted the oscillatory form, as a function of i $\Omega$.

$$
\begin{aligned}
& \text { ln } \operatorname{det} \mathcal{O}_{\beta}=2 \pi \Omega+4 \omega(\mathbb{K}-\mathbb{E}) \Omega^{-1}+\mathcal{O}\left(\Omega^{-3}\right), \\
& \ln \operatorname{det} \mathcal{O}_{\phi}=2 \pi \Omega+8 \omega(\mathbb{K}-\mathbb{E}) \Omega^{-1}+\mathcal{O}\left(\Omega^{-3}\right), \\
& \ln \operatorname{det} \mathcal{O}_{\psi}=2 \pi \Omega+2 \omega(\mathbb{K}-\mathbb{E}) \Omega^{-1}+\mathcal{O}\left(\Omega^{-3}\right), \\
& \ln \operatorname{det} \mathcal{O}_{0}=2 \pi \Omega+\mathcal{O}\left(\Omega^{-3}\right) .
\end{aligned}
$$

The leading (quadratically divergent) terms cancel in (5.1) due to the balance of world-sheet degrees of freedom in (5.1). The subleading (logarithmically divergent) terms also cancel in the combination appearing in $(5.1),(2 \times 4+8-8 \times 2)(\mathbb{K}-\mathbb{E}) \Omega^{-1}=0$. We thus confirm that the static-gauge result for the one-loop energy is indeed UV finite, as was argued in section 3.2.

\subsection{Equivalence between the static-gauge and conformal-gauge results}

To check the equivalence between the static-gauge and conformal-gauge results one needs to verify the factorization relation (3.17). This can be done numerically, as follows. To evaluate the left-hand side of (3.17) we used the Gel'fand-Yaglom method (for details, see appendix B) to compute numerically the determinant of the operator $\mathcal{O}_{t \rho \phi}$ in (3.7) as a function of $\Omega$ for various values of $k$. The right-hand side of (3.17) can be computed directly using the expression for the determinant of $\mathcal{O}_{\phi}$ found above (5.11). We find perfect agreement. In figure 6 we have plotted the expressions on both sides of (3.17) as functions of $\Omega$ for $k=\frac{1}{\sqrt{10}}$. Similar agreement is found for any $k$.

\subsection{General form of the one-loop correction $E_{1}$}

Going back to the complete expression for $E_{1}$ in (5.1) it is useful, in order to safely expand in one of the interesting limits analysed below, to separate there the contributions of the massless 


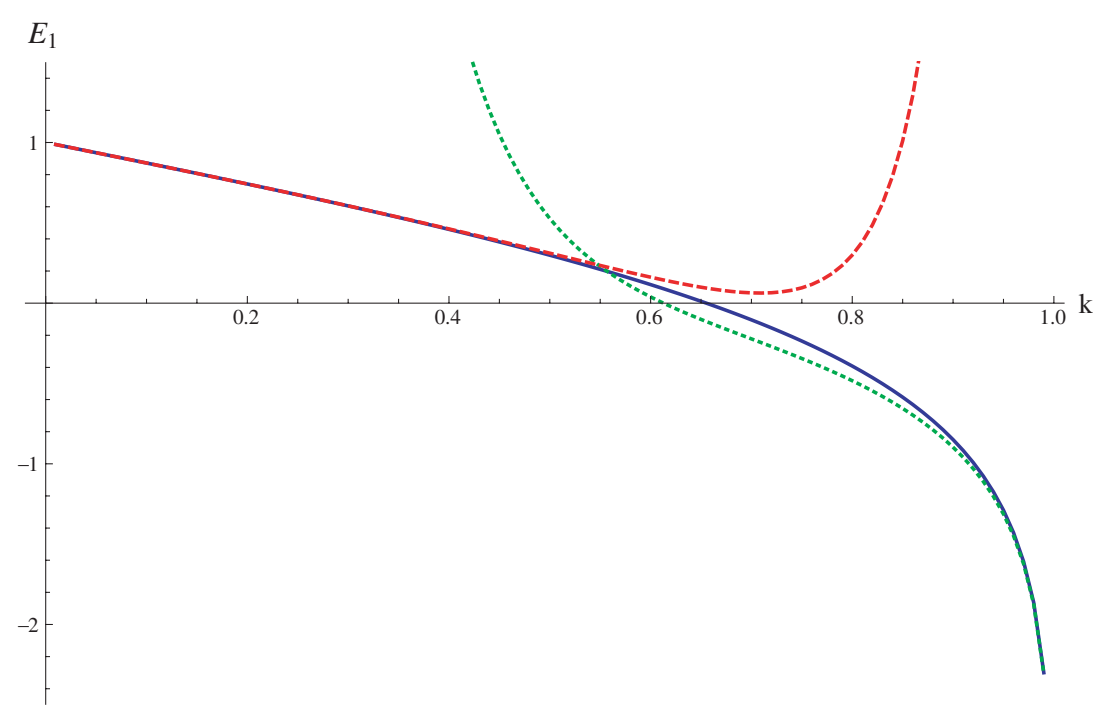

Figure 7. Plots of $E_{1}$ as a function of $k$ : the blue, solid, curve is found numerically from the exact expression (5.18) for generic values of $k$; the green, dotted, curve is found from an analytic expansion in the $k \rightarrow 1$ or large-spin limit, using the first two terms in (6.36); the red, dashed, curve is found from an analytic expansion in the $k \rightarrow 0$ or small-spin limit, using the first two terms in (7.7). The agreement is excellent in both extreme limits.

modes of $\mathcal{O}_{0}$ (i.e. $\left.\Omega^{2}\right)$ and the lowest eigenvalues $\left(\bar{\Lambda}_{1}\right.$ in table 1$)$ of $\mathcal{O}_{\beta}, \mathcal{O}_{\phi}$ and $\mathcal{O}_{\psi}$. Then, we get

$$
E_{1}=-\frac{1}{4 \pi \kappa} \int_{-\infty}^{\infty} \mathrm{d} \Omega\left[\ln \frac{\left(\operatorname{det}^{\prime} \mathcal{O}_{\psi}\right)^{8}}{\operatorname{det}^{\prime} \mathcal{O}_{\phi}\left(\operatorname{det}^{\prime} \mathcal{O}_{\beta}\right)^{2}\left(\operatorname{det}^{\prime} \mathcal{O}_{0}\right)^{5}}+h(\Omega)\right]
$$

where

$$
\begin{aligned}
& \operatorname{det}^{\prime} \mathcal{O}_{\beta, \phi, \psi} \equiv \frac{\operatorname{det} \mathcal{O}_{\beta, \phi, \psi}}{\bar{\Lambda}_{1}}, \quad \operatorname{det}^{\prime} \mathcal{O}_{0} \equiv \frac{\operatorname{det} \mathcal{O}_{0}}{\Omega^{2}} \\
& h(\Omega)=2 \ln \left(\Omega^{2}\right)-2 \ln \left(\Omega^{2}+\kappa^{2}\right) .
\end{aligned}
$$

Using that

$$
\int_{-\infty}^{\infty} \mathrm{d} \Omega h\left(\Omega^{2}\right)=-4 \pi \kappa
$$

the one-loop correction to the energy (5.1) takes the form

$$
E_{1}=1-\frac{1}{4 \pi \kappa} \int_{-\infty}^{\infty} \mathrm{d} \Omega \ln \frac{\left(\operatorname{det}^{\prime} \mathcal{O}_{\psi}\right)^{8}}{\operatorname{det}^{\prime} \mathcal{O}_{\phi}\left(\operatorname{det}^{\prime} \mathcal{O}_{\beta}\right)^{2}\left(\operatorname{det}^{\prime} \mathcal{O}_{0}\right)^{5}} .
$$

This expression is straightforward to evaluate numerically for various values of $k$ or the spin $\mathcal{S}$ in (2.11), and thus to plot $E_{1}$.

To gain more analytic control over the form of $E_{1}$ as a function of the spin $\mathcal{S}$ we may consider the expansion of it in the large-spin ('long string' or $k \rightarrow 1$ ) limit or in the small-spin ('short string' or $k \rightarrow 0$ ) limit. This will be done in detail in sections 6 and 7 respectively. In figure 7 we present together the results-the plots of $E_{1}(k)$ found analytically in the large-spin expansion (right-most green curve) and in the small-spin expansion (left-most red curve) and also the plot of the exact $E_{1}$ found numerically from (5.18) (blue curve connecting the two asymptotic ones). As one can see, already the few leading terms in the two respective analytic expansions give a very good approximation to the exact result. 


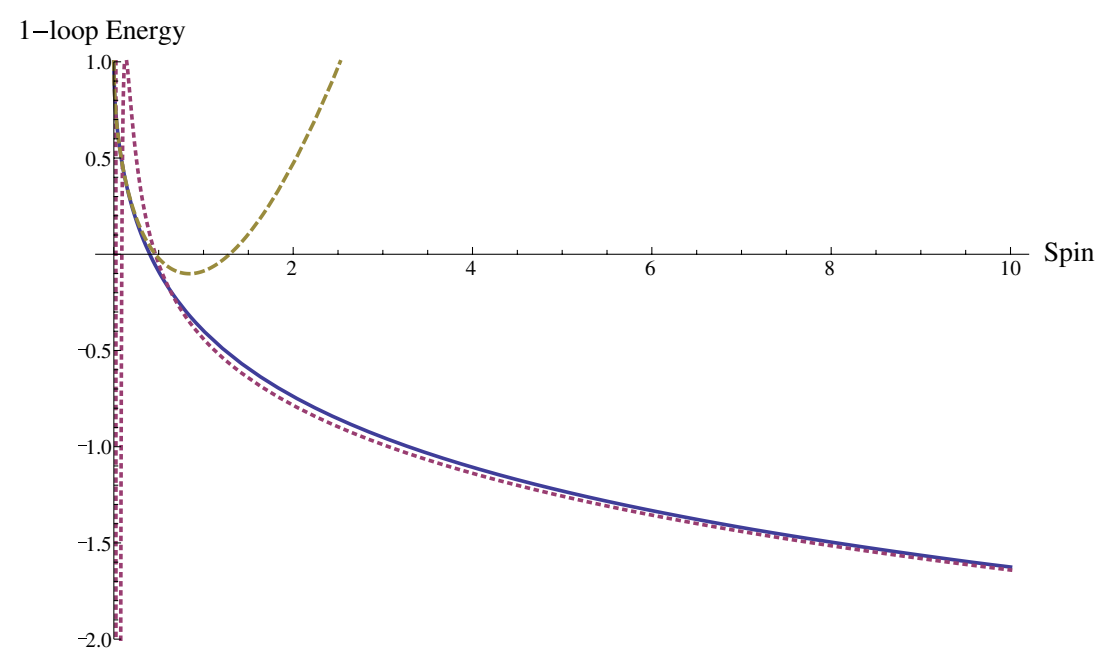

Figure 8. Plots of $E_{1}$ as a function of the classical spin $\mathcal{S}$. The solid, blue curve is the exact result, compared with the red (dotted) curve representing the large-spin expansion, see (6.41), and with the gold (dashed) curve representing the small-spin expansion found below in (7.11)-(7.12).

\section{Large-spin expansion}

This limit (see (2.12)) is defined as $k \rightarrow 1$ or, equivalently, $\eta \rightarrow 0$ in (2.5).

\subsection{Leading order}

In this subsection we will compute the leading term in the $k \rightarrow 1$ expansion of $E_{1}$ in (5.1) and also comment on first exponential subleading terms.

At the leading order the classical solution is approximated by

$$
\rho^{\prime} \approx \kappa_{0}, \quad \rho^{\prime \prime} \approx 0, \quad \omega \approx \kappa \approx \kappa_{0}, \quad \kappa_{0}=\frac{1}{\pi} \ln \frac{16}{\eta} \rightarrow \infty
$$

If we use these limiting expressions directly in the fluctuation operators, then we conclude that their potential terms become constant:

$\mathcal{O}_{\beta, 0}=-\partial_{\sigma}^{2}+2 \kappa_{0}^{2}+\Omega^{2}, \quad \mathcal{O}_{\phi, 0}=-\partial_{\sigma}^{2}+4 \kappa_{0}^{2}+\Omega^{2}, \quad \mathcal{O}_{\psi_{ \pm}, 0}=-\partial_{\sigma}^{2}+\kappa_{0}^{2}+\Omega^{2}$

and thus we find from (5.1) [3]

$$
E_{1}^{(0)}=\frac{1}{2 \kappa_{0}} \sum_{n=-\infty}^{\infty}\left[\sqrt{n^{2}+4 \kappa_{0}^{2}}+2 \sqrt{n^{2}+2 \kappa_{0}^{2}}+5 \sqrt{n^{2}}-8 \sqrt{n^{2}+\kappa_{0}^{2}}\right]
$$

where we performed the integration over $\Omega$ before commuting the determinants defined on a unit circle. Using the Euler-MacLaurin formula to transform the sum into an integral one finds

$$
E_{1}^{(0)}=\frac{1}{\kappa_{0}}\left[-3 \kappa_{0}^{2} \ln 2-\frac{5}{12}+\mathcal{O}\left(\mathrm{e}^{-2 \pi \kappa_{0}}\right)\right], \quad \kappa_{0} \rightarrow \infty,
$$

where the leading term is the result of [3] and the subleading term appeared in [22].

Let us now see what we get if we start instead with the exact expressions for the determinants (5.2)-(5.4). Using the expressions collected in appendix C (see (C.8)-(C.10)), we get at the leading order 


$$
\begin{aligned}
& 2 \mathbb{K}\left(k^{2}\right) Z\left(\alpha_{\beta} \mid k^{2}\right) \approx \pi \kappa_{0} x \quad \text { with } \quad x=\sqrt{2+\frac{\Omega^{2}}{\kappa_{0}^{2}}}, \\
& 2 \mathbb{K}\left(\tilde{k}^{2}\right) Z\left(\alpha_{\phi} \mid \tilde{k}^{2}\right) \approx \pi \kappa_{0} y \quad \text { with } \quad y=\sqrt{4+\frac{\Omega^{2}}{\kappa_{0}^{2}}}, \\
& \mathbb{K}\left(\tilde{k}^{2}\right) Z\left(\alpha_{\psi} \mid \tilde{k}^{2}\right) \approx \pi \kappa_{0} z \quad \text { with } \quad z=\sqrt{1+\frac{\Omega^{2}}{\kappa_{0}^{2}}},
\end{aligned}
$$

which would give, once substituted into (5.2)-(5.4), the following expressions for the determinants:

$$
\begin{aligned}
\operatorname{det} \mathcal{O}_{\beta}\left(\Omega^{2}\right) & \approx 4 \sinh ^{2}\left[\pi \kappa_{0} x\right] \\
\operatorname{det} \mathcal{O}_{\phi}\left(\Omega^{2}\right) & \approx 4 \sinh ^{2}\left[\pi \kappa_{0} y\right] \\
\operatorname{det} \mathcal{O}_{\psi}\left(\Omega^{2}\right) & \approx-4 \cosh ^{2}\left[\pi \kappa_{0} z\right] .
\end{aligned}
$$

Integrating logarithms of (6.8)-(6.10) over $\Omega$ one gets the result that may also be represented as

$$
E_{1} \approx \tilde{E}_{1}^{(0)}=\frac{1}{2 \kappa_{0}} \sum_{n=-\infty}^{\infty}\left[\sqrt{n^{2}+4 \kappa_{0}^{2}}+2 \sqrt{n^{2}+2 \kappa_{0}^{2}}+5 \sqrt{n^{2}}-8 \sqrt{\left(n+\frac{1}{2}\right)^{2}+\kappa_{0}^{2}}\right] .
$$

Here the shift $n \rightarrow n+\frac{1}{2}$ in the fermionic contribution is due to the $\cosh ^{2}$ instead of the $\sinh ^{2}$ form of the determinant (6.10). ${ }^{17}$ While this shift does not affect the result for the two leading terms in (6.4), it formally changes the form of the subleading corrections (which should not, however, be trusted in the approximation used to arrive at (6.11)).

Indeed, there is of course no contradiction as the approximation used to derive (6.4) was supposed to be valid only for the leading term in the large $\kappa_{0}$ expansion, i.e. the expressions for the subleading terms should not be trusted a priori. Still, let us briefly comment on the exponential corrections to the first two leading terms in (6.4) comparing what follows from (6.3) to what follows from (6.11). As was found in [27] using $\zeta$-function regularization of the sums in (6.3)

$E_{1}^{(0)}=-3 \kappa_{0} \ln 2-\frac{5}{12 \kappa_{0}}-\frac{1}{\pi} \sum_{n=1}^{\infty} \frac{1}{n}\left[K_{1}\left(4 \pi n \kappa_{0}\right)+\sqrt{2} K_{1}\left(2 \sqrt{2} \pi n \kappa_{0}\right)-4 K_{1}\left(2 \pi n \kappa_{0}\right)\right]$,

where $K_{1}$ is the Bessel function of the second type:

$$
\int_{m}^{\infty} \mathrm{d} x \sqrt{x^{2}-m^{2}} \mathrm{e}^{-2 \pi k x}=\frac{m}{2 \pi k} K_{1}(2 \pi k m) .
$$

The $K_{1}$ terms represent the exponential corrections since

$$
K_{1}(y) \rightarrow \sqrt{\frac{\pi}{2 y}} \mathrm{e}^{-y}\left[1+\mathcal{O}\left(y^{-1}\right)\right], \quad y \rightarrow \infty .
$$

17 This shift may be formally interpreted by saying that fermions have antiperiodic boundary conditions, so that $\ln \operatorname{det}\left(-\partial_{\sigma}^{2}+\Omega^{2}+\kappa_{0}^{2}\right)=\sum_{n=-\infty}^{+\infty} \ln \left[\left(n+\frac{1}{2}\right)^{2}+\omega^{2}+\kappa_{0}^{2}\right]$. This interpretation is more of a curiosity and should not be taken literally as this expression was derived in the large $\kappa_{0}$ limit where the distinction between the periodic and antiperiodic fermion boundary conditions is not actually visible. 
Repeating the same computation in the case of (6.11) one finds that

$$
\tilde{E}_{1}^{(0)}=E_{1}^{(0)}+\frac{4}{\pi} \sum_{n=1}^{\infty}\left[(-1)^{n}-1\right] K_{1}\left(2 \pi n \kappa_{0}\right) .
$$

\subsection{Beyond the leading order}

To find subleading corrections in large $\kappa$ let us add and subtract the leading-order contribution (6.4) from expression (5.1):

$$
\begin{aligned}
& E_{1}=\frac{1}{\kappa}\left[-3 \kappa_{0}^{2} \ln 2-\frac{5}{12}+\mathcal{O}\left(\eta^{2}\right)\right]+E_{1}^{(\mathrm{sub})}, \quad \kappa_{0} \rightarrow \infty \\
& E_{1}^{\text {(sub) }}=-\frac{\kappa_{0}}{4 \pi \kappa} \int_{-\infty}^{\infty} \mathrm{d} \bar{\Omega} \ln \frac{\mathcal{D}_{\psi}^{8}}{\mathcal{D}_{\beta}^{2} \mathcal{D}_{\phi}}
\end{aligned}
$$

Here we have defined the following ratios of determinants

$$
\mathcal{D}_{\beta}=\frac{\operatorname{det} \mathcal{O}_{\beta}}{\operatorname{det} \mathcal{O}_{\beta, 0}}, \quad \mathcal{D}_{\phi}=\frac{\operatorname{det} \mathcal{O}_{\phi}}{\operatorname{det} \mathcal{O}_{\phi, 0}}, \quad \mathcal{D}_{\psi}=\frac{\operatorname{det} \mathcal{O}_{\psi}}{\operatorname{det} \mathcal{O}_{\psi, 0}}
$$

and introduced $\bar{\Omega}=\frac{\Omega}{\kappa_{0}}$ which is the argument the integrand according to (6.5) $-(6.7)$.

Expanding the arguments of the determinants, one finds (see (C.8)-(C.10))

$$
\begin{aligned}
& 2 \mathbb{K} Z\left(\alpha_{\beta} \mid k^{2}\right) \approx \pi \kappa_{0} x-2 \tanh ^{-1} x \\
& 2 \widetilde{\mathbb{K}} Z\left(\alpha_{\phi} \mid \tilde{k}^{2}\right) \approx \pi \kappa_{0} y-2 \tanh ^{-1} \frac{y}{2} \\
& \widetilde{\mathbb{K}} Z\left(\alpha_{\psi} \mid \tilde{k}^{2}\right) \approx \pi \kappa_{0} z-\tanh ^{-1} z
\end{aligned}
$$

and therefore

$$
\begin{aligned}
& \mathcal{D}_{\beta}=\frac{\sinh ^{2}\left[2 \mathbb{K} Z\left(\alpha_{\beta} \mid k^{2}\right)\right]}{\sinh ^{2}\left[\pi \kappa_{0} x\right]} \approx\left[\frac{x^{2}+1}{x^{2}-1}-\frac{2 x}{x^{2}-1} \operatorname{coth}\left(\pi \kappa_{0} x\right)\right]^{2} \\
& \mathcal{D}_{\phi}=\frac{\sinh ^{2}\left[2 \widetilde{\mathbb{K}} Z\left(\alpha_{\phi} \mid \tilde{k}^{2}\right)\right]}{\sinh ^{2}\left[\pi \kappa_{0} y\right]} \approx\left[\frac{y^{2}+4}{y^{2}-4}-\frac{4 y}{y^{2}-4} \operatorname{coth}\left(\pi \kappa_{0} y\right)\right]^{2} \\
& \mathcal{D}_{\psi}=\frac{\cosh ^{2}\left[\widetilde{\mathbb{K}} Z\left(\alpha_{\psi} \mid \tilde{k}^{2}\right)\right]}{\cosh ^{2}\left[\pi \kappa_{0} z\right]} \approx \frac{1}{1-z^{2}}\left[1-z \tanh \left(\pi \kappa_{0} z\right)\right]^{2} .
\end{aligned}
$$

Neglecting the tanh and coth terms in the square brackets for large $\kappa_{0}$ one finds that the second contribution in (6.16), first contribution at next-to-leading order, results in

$$
\begin{aligned}
E_{1}^{(\mathrm{sub})} & \approx-\frac{\kappa_{0}}{4 \pi \kappa} \int_{-\infty}^{+\infty} \mathrm{d} \bar{\Omega} \ln \left[\left(\frac{1-\sqrt{1+\bar{\Omega}^{2}}}{1+\sqrt{1+\bar{\Omega}^{2}}}\right)^{8}\left(\frac{1-\sqrt{2+\bar{\Omega}^{2}}}{1+\sqrt{2+\bar{\Omega}^{2}}}\right)^{-4}\left(\frac{2-\sqrt{4+\bar{\Omega}^{2}}}{2+\sqrt{4+\bar{\Omega}^{2}}}\right)^{-1}\right] \\
& =1+\frac{6}{\pi} \ln 2, \quad \kappa_{0} \rightarrow \infty,
\end{aligned}
$$

where we set $\kappa \approx \kappa_{0}$. The same result for this subleading coefficient was found in [24] using the integrability (algebraic curve) approach (see also [10] and [7]). As discussed in [10] this correction should be due to the near turning point contribution that is lost in the naive approach that treats the potential terms perturbatively. 
Proceeding to the next order $\sim \eta=k^{-2}-1$, the evaluation of the various functional determinant ratios gives

$$
\begin{aligned}
& \mathcal{D}_{\beta}=\frac{(x-1)^{2}}{(x+1)^{2}}\left[1+\frac{2}{x} \eta-\frac{\eta}{\pi \kappa_{0}} \frac{2\left(x^{2}-2\right)}{x\left(x^{2}-1\right)}+\mathcal{O}\left(\eta^{2}\right)\right], \\
& \mathcal{D}_{\phi}=\frac{(y-2)^{2}}{(y+2)^{2}}\left[1+\frac{4}{y} \eta-\frac{\eta}{\pi \kappa_{0}} \frac{4}{y}+\mathcal{O}\left(\eta^{2}\right)\right], \\
& \mathcal{D}_{\psi}=\frac{1-z}{1+z}\left[1+\frac{1}{z} \eta-\frac{\eta}{\pi \kappa_{0}} \frac{1}{z}+\mathcal{O}\left(\eta^{2}\right)\right],
\end{aligned}
$$

so that

$$
\ln \frac{\mathcal{D}_{\psi}^{8}}{\mathcal{D}_{\beta}^{2} \mathcal{D}_{\phi}}=e(\bar{\Omega})+\eta\left[f(\bar{\Omega})+\frac{g(\bar{\Omega})}{\pi \kappa_{0}}\right]+\cdots,
$$

where $e(\bar{\Omega})$ is the integrand in (6.25). The functions $f(\bar{\Omega})$ and $g(\bar{\Omega})$ are

$$
\begin{aligned}
& f(\bar{\Omega})=\frac{8}{\sqrt{1+\bar{\Omega}^{2}}}-\frac{4}{\sqrt{2+\bar{\Omega}^{2}}}-\frac{4}{\sqrt{4+\bar{\Omega}^{2}}} \\
& g(\bar{\Omega})=-\frac{8}{\sqrt{1+\bar{\Omega}^{2}}}+\frac{4 \bar{\Omega}^{2}}{\left(1+\bar{\Omega}^{2}\right) \sqrt{2+\bar{\Omega}^{2}}}+\frac{4}{\sqrt{4+\bar{\Omega}^{2}}}
\end{aligned}
$$

and their integrals take the values

$$
\int_{-\infty}^{+\infty} \mathrm{d} \bar{\Omega} f(\bar{\Omega})=12 \ln 2, \quad \int_{-\infty}^{+\infty} \mathrm{d} \bar{\Omega} g(\bar{\Omega})=-2(\pi+6 \ln 2) .
$$

We conclude that to order $\eta$ the large $\kappa$ expansion of the one-loop energy reads

$$
\begin{aligned}
E_{1}=\frac{1}{\kappa}\left[-3 \kappa_{0}^{2}\right. & \ln 2-\frac{5}{12}+\left(1+\frac{6}{\pi} \ln 2\right) \kappa_{0} \\
- & \left.\frac{1}{\pi}\left[3 \kappa_{0} \ln 2-\frac{1}{2}\left(1+\frac{6}{\pi} \ln 2\right)\right] \eta+\mathcal{O}\left(\eta^{2}\right)\right], \quad \kappa_{0} \rightarrow \infty
\end{aligned}
$$

Here we did not expanded explicitly the overall factor of $\frac{1}{\kappa}$,

$$
\frac{1}{\kappa}=\frac{1}{\kappa_{0}}\left[1+\frac{1}{4}\left(1-\frac{2}{\pi \kappa_{0}}\right) \eta+\mathcal{O}\left(\eta^{2}\right)\right], \quad \eta=16 \mathrm{e}^{-\pi \kappa_{0}} \rightarrow 0
$$

The coefficient of the leading $\eta$ correction is in agreement with the one found in [10], while the next $\frac{\eta}{\kappa_{0}}$ term is a new result.

In going to higher than first orders of expansion in $\eta$ there is a potential problem of accounting for the contributions of terms like $\operatorname{coth}\left(\pi \kappa_{0} x\right)-1$ in (6.22)-(6.24) we have dropped above. For example,

$$
\mathrm{e}^{-2 \kappa_{0} \pi z} \sim\left(\frac{\eta}{16}\right)^{2}\left[1-\frac{\pi \Omega^{2}}{\kappa_{0}}+\frac{\pi^{2} \Omega^{4}}{2 \kappa_{0}^{2}}+\frac{3 \pi^{4} \Omega^{4}-2 \pi^{6} \Omega^{6}}{12 \kappa_{0}^{3} \pi^{3}}+\cdots\right],
$$

and similar terms also arise in the expansion of the reference determinants, see (6.14). Such terms need to be resummed, and, while there is the possibility that all such terms may cancel, this is not clear at the moment. In appendix D we present the evaluation of the leading large $\kappa_{0}$ correction to the one-loop energy due to these contributions, while in appendix E we consider a different type of expansion in the $k \rightarrow 1$ limit. 
Ignoring this complication, we have found that the one-loop energy has the following structure of large-spin expansion:

$$
\begin{aligned}
E_{1}=\frac{\kappa_{0}}{\kappa}\left[\left(c_{01} \kappa_{0}+c_{00}+\frac{c_{0,-1}}{\kappa_{0}}\right)+\left(c_{11} \kappa_{0}+c_{10}+\frac{c_{1,-1}}{\kappa_{0}}\right) \eta\right. \\
\left.\quad+\left(c_{21} \kappa_{0}+c_{20}+\frac{c_{2,-1}}{\kappa_{0}}\right) \eta^{2}+\left(c_{31} \kappa_{0}+c_{30}+\frac{c_{3,-1}}{\kappa_{0}}\right) \eta^{3}+\mathcal{O}\left(\eta^{4}\right)\right]
\end{aligned}
$$

where the explicit values are

$$
\begin{aligned}
& c_{01}=-3 \ln 2, \quad c_{00}=1+\frac{6}{\pi} \ln 2 \quad c_{0,-1}=-\frac{5}{12}, \\
& c_{11}=0, \quad c_{10}=-\frac{3}{\pi} \ln 2 \quad c_{1,-1}=\frac{1}{2 \pi}+\frac{3 \ln 2}{\pi^{2}}, \\
& c_{21}=-\frac{\pi}{32}-\frac{3}{32} \ln 2, \quad c_{20}=\frac{1}{16}+\frac{39 \ln 2}{32 \pi}, \quad c_{2,-1}=-\frac{13}{64 \pi}-\frac{63 \ln 2}{32 \pi^{2}}, \\
& c_{31}=\frac{\pi}{32}+\frac{3}{32} \ln 2, \quad c_{30}=-\frac{3}{32}-\frac{13 \ln 2}{16 \pi}, \quad c_{3,-1}=\frac{29}{192 \pi}+\frac{85 \ln 2}{64 \pi^{2}} .
\end{aligned}
$$

For completeness, we report here the first few orders in the large-spin expansion of the one-loop energy as found using (2.12) in (6.36):

$$
\begin{aligned}
E_{1}=-\frac{3 \ln 2}{\pi} & \ln \overline{\mathcal{S}}+\frac{\pi+6 \ln 2}{\pi}-\frac{5 \pi}{12 \ln \overline{\mathcal{S}}} \\
& -\frac{1}{\overline{\mathcal{S}}}\left[\frac{24 \ln 2}{\pi} \ln \overline{\mathcal{S}}-\frac{4 \pi+36 \ln 2}{\pi}+\frac{5 \pi}{3 \ln ^{2} \overline{\mathcal{S}}}\right]+\mathcal{O}\left(\frac{1}{\overline{\mathcal{S}}^{2}}\right)
\end{aligned}
$$

$\overline{\mathcal{S}}=8 \pi \mathcal{S}, \quad \mathcal{S} \gg 1$

\subsection{Test of reciprocity}

With expressions (6.36)-(6.40) at hand, we are able to the confirm and extend the analysis of [10], in which the reciprocity relations between the coefficients in the large-spin expansion of the energy (or twist 2 anomalous dimension at strong coupling) [8, 32, 33] were checked up to order $\eta$.

To do this one needs to determine the functions ${ }^{18}$

$\Delta(\mathcal{S})=\Delta_{0}+\frac{1}{\sqrt{\lambda}} \Delta_{1}+\cdots, \quad \Delta_{0}=\mathcal{E}_{0}(\mathcal{S})-\mathcal{S}, \quad \Delta_{1}=\mathcal{E}_{1}(\mathcal{S})$,

as the functions of the spin $\mathcal{S}$, which as at the classical level is obtained by replacing the parameter $\eta$ with its expansion $\eta=\eta(\mathcal{S})$ in terms of the spin (2.14). One is then to compute the function $\mathcal{P}$ defined by

$$
\Delta(\mathcal{S})=\mathcal{P}\left(\mathcal{S}+\frac{1}{2} \Delta(\mathcal{S})\right)
$$

The test of reciprocity amounts to the check of parity of $\mathcal{P}(\mathcal{S})$ under $\mathcal{S} \rightarrow-\mathcal{S}$. Solving the functional equation in $(6.43)$ as

$$
\mathcal{P}(\mathcal{S})=\sum_{k=1}^{\infty} \frac{1}{k !}\left(-\frac{1}{2} \frac{\mathrm{d}}{\mathrm{d} \mathcal{S}}\right)^{k-1}[\Delta(\mathcal{S})]^{k}
$$

${ }^{18} \mathcal{E}_{0}$ and $\mathcal{E}_{1}$ are the classical and the one-loop energies rescaled by a factor string tension. 
and expanding the function $\mathcal{P}$ in $\frac{1}{\sqrt{\lambda}}$

$$
\mathcal{P}=\mathcal{P}_{0}+\frac{1}{\sqrt{\lambda}} \mathcal{P}_{1}+\cdots,
$$

one finds

$$
\begin{aligned}
& \mathcal{P}_{0}(\mathcal{S})=\sum_{k=1}^{\infty} \frac{1}{k !}\left(-\frac{1}{2} \frac{\mathrm{d}}{\mathrm{d} \mathcal{S}}\right)^{k-1}\left[\Delta_{0}(\mathcal{S})\right]^{k} \\
& \mathcal{P}_{1}(\mathcal{S})=\sum_{k=1}^{\infty} \frac{1}{k !}\left(-\frac{1}{2} \frac{\mathrm{d}}{\mathrm{d} \mathcal{S}}\right)^{k-1}\left[k \Delta_{0}(\mathcal{S})^{k-1} \Delta_{1}(\mathcal{S})\right]
\end{aligned}
$$

Working out $\mathcal{P}_{1}$ and looking at all terms which are odd under $\mathcal{S} \rightarrow-\mathcal{S}$ we find that they vanish if the following reciprocity constraints hold:

$$
\begin{aligned}
& c_{10}=\frac{1}{\pi} c_{01}, \quad c_{1,-1}=\frac{1}{2 \pi} c_{00}, \quad c_{31}=-c_{21}, \\
& c_{30}=-c_{20}-\frac{1}{6 \pi} c_{01}+\frac{1}{\pi} c_{21}, \\
& c_{3,-1}=-c_{2,-1}+\frac{1}{4 \pi^{2}} c_{01}-\frac{1}{12 \pi} c_{00}+\frac{1}{2 \pi} c_{20} .
\end{aligned}
$$

As usual, the coefficients of terms with odd powers of $\eta=\frac{2}{\mathcal{S}}+\cdots$ in (2.12) are determined by coefficients of terms with even powers of $\eta$. Using the list of explicit coefficients found above (6.37)-(6.40), we find that these relations are indeed satisfied.

\section{Small-spin expansion}

The small-spin or short-string limit $[9,14]$ is realized by sending $\eta \rightarrow \infty$ or $k \rightarrow 0$ (see section 2).

The general expansion of the determinants, see (C.13)-(C.18), has the form

$$
\operatorname{det} \mathcal{O}_{f}=D_{f}^{(0)}(\Omega)+\frac{1}{\eta} D_{f}^{(1)}(\Omega)+\frac{1}{\eta^{2}} D_{f}^{(2)}(\Omega)+\cdots, \quad f=(\beta, \phi, \psi),
$$

where

$$
D_{\beta}^{(0)}(\Omega)=D_{\phi}^{(0)}(\Omega)=D_{\psi}^{(0)}(\Omega)=4 \sinh ^{2}(\pi \Omega)
$$

$D_{\beta}^{(1)}(\Omega)=\frac{2 \pi \sinh (2 \pi \Omega)}{\Omega}, \quad D_{\phi}^{(1)}(\Omega)=\frac{4 \pi \Omega \sinh (2 \pi \Omega)}{\Omega^{2}+1}, \quad D_{\psi}^{(1)}(\Omega)=\frac{4 \pi \Omega \sinh (2 \pi \Omega)}{4 \Omega^{2}+1}$,

$D_{\beta}^{(2)}(\Omega)=\frac{\pi^{2} \cosh (2 \pi \Omega)}{\Omega^{2}}-\frac{\pi\left(3 \Omega^{4}+6 \Omega^{2}+2\right) \sinh (2 \pi \Omega)}{4\left(\Omega^{5}+\Omega^{3}\right)}$

$D_{\phi}^{(2)}(\Omega)=\frac{4 \pi^{2} \Omega^{2} \cosh (2 \pi \Omega)}{\left(\Omega^{2}+1\right)^{2}}-\frac{\pi \Omega\left(3\left(\Omega^{2}+4\right) \Omega^{2}+1\right) \sinh (2 \pi \Omega)}{2\left(\Omega^{2}+1\right)^{3}}$,

$D_{\psi}^{(2)}(\Omega)=-\frac{\pi \Omega\left(48\left(\Omega^{4}+\Omega^{2}\right)+1\right) \sinh (2 \pi \Omega)}{2\left(4 \Omega^{2}+1\right)^{3}}+\frac{4 \pi^{2} \Omega^{2} \cosh (2 \pi \Omega)}{\left(4 \Omega^{2}+1\right)^{2}}$.

The first correction to the quantity entering the effective action (5.1) is

$$
\ln \frac{\operatorname{det}^{8} \mathcal{O}_{\psi}}{\operatorname{det}_{\phi} \operatorname{det}^{2} \mathcal{O}_{\beta} \operatorname{det}^{5} \mathcal{O}_{0}}=\frac{1}{\eta} \frac{2 \pi\left(2 \Omega^{2}-1\right) \operatorname{coth}(\pi \Omega)}{\Omega\left(\Omega^{2}+1\right)\left(4 \Omega^{2}+1\right)}+\mathcal{O}\left(\frac{1}{\eta^{2}}\right)
$$


which is integrable at $\Omega \rightarrow \infty$ but has a pole at $\Omega=0$, i.e. produces an IR divergence. Such an IR effect disappears by integrating separately the lowest eigenvalues (see table 1), which, in fact, behave as zero modes around $\Omega \sim 0$ (in the case of the $\beta$ fluctuation this only happens in the short-string limit $\eta \rightarrow \infty)$ :

$$
\bar{\Lambda}_{1}^{(\beta)}=\Omega^{2}+\frac{1}{\eta}+\cdots, \quad \bar{\Lambda}_{1}^{(\phi)}=\Omega^{2}, \quad \bar{\Lambda}_{1}^{(\psi)}=\Omega^{2} .
$$

This is equivalent to use definition (5.18) for the one-loop correction to the energy. Indeed, with definition (5.15) the quantity one is to evaluate

$$
\ln \frac{\left(\operatorname{det}^{\prime} \mathcal{O}_{\psi}\right)^{8}}{\operatorname{det}^{\prime} \mathcal{O}_{\phi}\left(\operatorname{det}^{\prime} \mathcal{O}_{\beta}\right)^{2}\left(\operatorname{det}^{\prime} \mathcal{O}_{0}\right)^{5}}=\frac{1}{\eta} \frac{2\left[4 \Omega^{4}+5 \Omega^{2}+\pi\left(2 \Omega^{2}-1\right) \Omega \operatorname{coth}(\pi \Omega)+1\right]}{\Omega^{2}\left(4 \Omega^{4}+5 \Omega^{2}+1\right)}+\cdots
$$

is now finite and can be integrated to give $2 \pi(8 \ln 2-3)$. On the other hand, the contribution of the lowest eigenvalues has been shown to give a finite number at (5.17) at all orders in $1 / \eta$.

Going to one further order in the large $\eta$ expansions of the determinants and adding all together one finds for the expansion of the one-loop energy (5.18)

$$
\begin{aligned}
E_{1}= & 1-\frac{1}{4 \pi \kappa} \int_{-\infty}^{\infty} \mathrm{d} \Omega \ln \frac{\left(\operatorname{det}^{\prime} \mathcal{O}_{\psi}\right)^{8}}{\left(\operatorname{det}^{\prime} \mathcal{O}_{\beta}\right)^{2} \operatorname{det}^{\prime} \mathcal{O}_{\phi}\left(\operatorname{det}^{\prime} \mathcal{O}_{0}\right)^{5}} \\
= & 1+\frac{1}{\kappa}\left[\left(\frac{3}{2}-4 \ln 2\right) \eta^{-1}-\left(1-\frac{3}{2} \ln 2-\frac{3}{8} \zeta(3)\right) \eta^{-2}\right. \\
& \left.-\left(-\frac{27}{16}+\frac{7}{4} \ln 2+\frac{9}{32} \zeta(3)+\frac{15}{32} \zeta(5)\right) \eta^{-3}+\mathcal{O}\left(\eta^{4}\right)\right]
\end{aligned}
$$

Here we did not expand explicitly the factor

$$
\frac{1}{\kappa}=\sqrt{\eta}\left[1+\frac{1}{4} \eta^{-1}+\mathcal{O}\left(\eta^{-2}\right)\right] \text {. }
$$

Substituting the expansion of $\eta$ in terms of the spin (2.14), we can finally obtain the following small-spin expansion of the one-loop correction to the energy:

$$
\begin{aligned}
E_{1}=E_{1}^{(\text {an })}+ & E_{1}^{(\text {nan })}, \\
E_{1}^{(\text {an })}=\sqrt{2 \mathcal{S}}( & {\left[\frac{3}{2}-4 \ln 2\right]+\left[-\frac{23}{16}+\frac{3}{2} \ln 2+\frac{3}{4} \zeta(3)\right] \mathcal{S} } \\
& \left.+\left[\frac{689}{256}-\frac{63}{32} \ln 2-\frac{15}{32} \zeta(3)-\frac{15}{16} \zeta(5)\right] \mathcal{S}^{2}+\mathcal{O}\left(\mathcal{S}^{3}\right)\right),
\end{aligned}
$$

$E_{1}^{(\text {nan })}=1+\mathcal{O}(\mathcal{S})$.

We have separated $E_{1}$, as in [14], into an 'analytic' part (with $\mathcal{S}$-dependence similar to the classical energy (2.15)) and a 'non-analytic' part, containing 'would-be IR singular' contributions of the lowest eigenvalues.

We conclude that the procedure adopted in this paper leads to the same structure of the small-spin expansion of the one-loop energy as found in [9]. The coefficients of the transcendental terms proportional to $\ln 2$ and $\zeta(3)$ in (7.11) are exactly the same as in [9] (see equation (4.37) there). The coefficients of the rational terms are, however, different.

Let us note that a separate treatment of the zero-mode contribution in [34] also led to a different result for (7.12) (cited in equation (3.60) in [14]). References [9, 34] used the standard 'near-flat-space' perturbation theory treatment of the determinants in the conformal gauge. A disagreement with our present results is apparently due to the prescription adopted in $[9,34]$ for projecting out the zero mode contributions. As discussed in appendix F, a somewhat different prescription would lead to the same result as the one that one obtains using the small-spin perturbation theory for the static-gauge determinants. 


\section{Conclusions}

In this paper we have found an exact expression for the one-loop correction to the energy of the folded string spinning in the $\mathrm{AdS}_{3}$ part of $\mathrm{AdS}_{5} \times S^{5}$. The main technical advancement is that we have shown that all the fluctuation operators, in the static gauge, have the single-gap Lamé form. As a result, their determinants can be computed in a closed form. We have verified explicitly that, as expected, the one-loop energy correction is the same in the static and the conformal gauges, even though the structure of the two fluctuation determinant ratios appears to be quite different.

The analytic expressions for the fluctuation determinants permitted us to carry out improved expansions in the small- and large-spin limit; the latter allowed us to verify that the reciprocity relations continue to be satisfied at strong coupling. Perhaps more importantly, our demonstration that the semi-classical fluctuation problem is governed by simple finitegap operators gives a new perspective on the role of integrable systems in the analysis of quantum corrections in such string models. In fact, finite-gap fluctuation operators are naturally described in terms of the algebraic curves of Riemann surfaces associated with the finite-gap spectrum [36], making the connection with the classical integrability (algebraic curve) approach of $[15,16]$ explicit.

The integrability approach to semi-classical quantization relies on the classical integrable structure of the theory. The investigation of the monodromy of the Lax connection [37] for the $\mathrm{AdS}_{5} \times S^{5}$ superstring action leads to the derivation of a spectral curve for any solution of the classical string equations of motion [15]. This is an example of the general finite gap description of classically integrable theories [38] which, reformulated in terms of a RiemannHilbert problem, leads to certain integral equations for each finite-gap curve associated with a classical solution. The same finite-gap integral equations happen to appear in the continuum limit of the (discrete) algebraic Bethe ansatz equations [39].

Starting with the classical algebraic curve describing a particular solution one can develop a semiclassical quantization [16, 40] by deforming the cuts defining the algebraic curve (adding extra roots) [41, 42]. Fluctuations are then perturbations of the cuts, and the one-loop correction to the energy is given as usual by the sum of the energy shifts (or characteristic frequencies) due to these fluctuations. Alternatively, one may try to guess the quantum extension of the classical finite gap integral equations, having as the guiding principle the gauge-theory information implying a description in terms of an asymptotic Bethe ansatz [39]. Improved by the phase [43, 44] extracted from the one-loop string data of [45], the Bethe ansatz result for the one-loop correction to string energy was shown [46] to agree, for a generic classical superstring solution, with the approach based on extracting the characteristic frequencies by perturbing the algebraic curve. This general equivalence was recently extended to also include the exponentially suppressed finite size effects with the asymptotic Bethe ansatz starting point replaced by an appropriate thermodynamic Bethe ansatz (see [17] and references therein).

Comparing this integrability approach to the one of the present paper, note that even if we did not explicitly refer to the classical integrability of the string sigma model, we 'rediscovered' the integrability at the one-loop level via the connection with the integrable, finite-gap, Lamé equation.

In addition to stimulating the study of detailed relation between the two approaches at the one-loop level [18], the findings of the present papers have a methodological merit of explicitly illustrating on a rather important and non-trivial example of how the integrability of the $\mathrm{AdS}_{5} \times S^{5}$ superstring sigma model is extended from the classical to the semiclassical one-loop level. 
This connection is, of course, not surprising from a general perspective: given a set of integrable classical equations, the linear problem for small fluctuations near a given solution is found by considering a small variation of the original nonlinear equations and should thus be essentially controlled by the original classical integrable structure. However, the technical details of such connection may be quite intricate. The small fluctuation problem is, in general, described by a complicated coupled set of linear differential equations, i.e. by a matrix differential operator, while the standard examples of integrable spectral problems involve second-order ordinary differential operators with their integrability related to a special type of their potential terms. The general study of which kind of integrable matrix differential operator spectral problems are associated with nonlinear string sigma model type classical equations appears to be an interesting open problem.

The extension of our present results to the case of a spinning folded string with a non-zero angular momentum in $S^{5}$ is currently under investigation [18].

\section{Acknowledgments}

We thank I Adam, T McLaughlin, C Meneghelli, R Roiban, D Seminara, S Theisen, A Tirziu and especially $\mathrm{N}$ Gromov for useful discussions. We are grateful to R Roiban for helpful comments on the draft. VF would like to thank the Theory Groups of IPhT CEA-Saclay and DESY-Hamburg for discussions and kind hospitality while parts of this work were done. GD is supported in part by the DOE grant DE-FG02-92ER40716. VF is supported by the Alexander von Humboldt foundation.

\section{Appendix A. Relevant elliptic-function properties and identities}

\section{A.1. Complete elliptic integrals}

The complete elliptic integrals of the first and second kind are defined as functions of their modulus $k^{2}$ as follows:

$\mathbb{K}\left(k^{2}\right)=\mathbb{K}=\int_{0}^{\frac{\pi}{2}} \mathrm{~d} \theta\left(1-k^{2} \sin ^{2} \theta\right)^{-1 / 2}, \quad \mathbb{E}\left(k^{2}\right)=\mathbb{E}=\int_{0}^{\frac{\pi}{2}} \mathrm{~d} \theta\left(1-k^{2} \sin ^{2} \theta\right)^{1 / 2}$

One also defines the complementary modulus

$k^{\prime 2}=1-k^{2} \quad$ and $\quad \mathbb{K}^{\prime}\left(k^{2}\right)=\mathbb{K}^{\prime}=\mathbb{K}\left(1-k^{2}\right)=\int_{0}^{\frac{\pi}{2}} \mathrm{~d} \theta\left(1-k^{\prime 2} \sin ^{2} \theta\right)^{-1 / 2}$

\section{A.2. Jacobi elliptic functions}

Defining the Jacobi amplitude as

$\varphi=\operatorname{am}\left(u \mid k^{2}\right), \quad$ where $\quad u=\int_{0}^{\varphi} \mathrm{d} \theta\left(1-k^{\prime 2} \sin ^{2} \theta\right)^{-1 / 2}$

the Jacobi elliptic functions sn, cn, dn defined by

$\operatorname{sn}\left(u \mid k^{2}\right)=\sin \varphi, \quad \operatorname{cn}\left(u \mid k^{2}\right)=\cos \varphi, \quad \operatorname{dn}\left(u \mid k^{2}\right)=\left(1-k^{2} \sin ^{2} \varphi\right)^{1 / 2}$

are doubly periodic functions of $u$, with real-valued periods that are either $2 \mathbb{K}(\mathrm{dn})$ or $4 \mathbb{K}$ (sn and cn) and purely imaginary periods that are either $2 \mathrm{i} \mathbb{K}^{\prime}(\mathrm{sn})$ or $4 \mathrm{i} \mathbb{K}^{\prime}$ (cn and dn). The fundamental period-parallelogram for the Jacobi elliptic functions is, therefore, the rectangle with corners at $\left(0,4 \mathbb{K}, 4 \mathrm{i} \mathbb{K}^{\prime}, 4 \mathbb{K}+4 \mathrm{i} \mathbb{K}^{\prime}\right)$, where zero occurs for real values of $u$ (at $2 \mathbb{K}$ and $4 \mathbb{K}$ ) while singularities occur for imaginary values of $u$ (at $\mathrm{i} \mathbb{K}^{\prime}$ and $3 i \mathbb{K}^{\prime}$ ). 
Other Jacobian elliptic functions useful for us are

$$
\begin{array}{ll}
\operatorname{cd}\left(u \mid k^{2}\right)=\frac{\operatorname{cn}\left(u \mid k^{2}\right)}{\operatorname{dn}\left(u \mid k^{2}\right)}, & \operatorname{sd}\left(u \mid k^{2}\right)=\frac{\operatorname{sn}\left(u \mid k^{2}\right)}{\operatorname{dn}\left(u \mid k^{2}\right)} \\
\operatorname{ns}\left(u \mid k^{2}\right)=\frac{1}{\operatorname{sn}\left(u \mid k^{2}\right)}, & \operatorname{nd}\left(u \mid k^{2}\right)=\frac{1}{\operatorname{dn}\left(u \mid k^{2}\right)} .
\end{array}
$$

Useful relations between the squares of the functions are

$$
\begin{aligned}
& -\operatorname{dn}^{2}\left(u \mid k^{2}\right)+k^{\prime 2}=-k^{2} \operatorname{cn}^{2}\left(u \mid k^{2}\right)=k^{2} \operatorname{sn}^{2}\left(u \mid k^{2}\right)-k^{2} \\
& -k^{\prime 2} \operatorname{nd}\left(u \mid k^{2}\right)+k^{\prime 2}=-k^{2} k^{\prime 2} \operatorname{sd}^{2}\left(u \mid k^{2}\right)=k^{2} \operatorname{cd}\left(u \mid k^{2}\right)-k^{2} .
\end{aligned}
$$

A useful representation for $\operatorname{sn}\left(u \mid k^{2}\right)$ is

$$
\operatorname{sn}\left(u \mid k^{2}\right)=\frac{\pi}{2 \mathbb{K}^{\prime}} \sum_{n=-\infty}^{\infty}(-1)^{n} \tanh \left(\frac{\pi}{2 \mathbb{K}^{\prime}}(u-2 n \mathbb{K})\right) .
$$

\section{A.3. Jacobi eta, theta and zeta functions}

The Jacobi $H, \Theta$ and $Z$ functions are defined as follows in terms of the Jacobi $\vartheta$ functions:

$H\left(u \mid k^{2}\right)=\vartheta_{1}\left(\frac{\pi u}{2 \mathbb{K}}, q\right), \quad \Theta\left(u \mid k^{2}\right)=\vartheta_{4}\left(\frac{\pi u}{2 \mathbb{K}}, q\right), \quad Z\left(u \mid k^{2}\right)=\frac{\pi}{2 \mathbb{K}} \frac{\vartheta_{4}^{\prime}\left(\frac{\pi u}{2 \mathbb{K}}, q\right)}{\vartheta_{4}\left(\frac{\pi u}{2 \mathbb{K}}, q\right)}$

where

$$
q=q\left(k^{2}\right)=\exp \left(-\pi \frac{\mathbb{K}^{\prime}}{\mathbb{K}}\right)
$$

Useful periodicities for them are

$$
\begin{aligned}
& H\left(u+2 \mathbb{K} \mid k^{2}\right)=-H\left(u \mid k^{2}\right), \\
& \Theta\left(u+2 \mathbb{K} \mid k^{2}\right)=\Theta\left(u \mid k^{2}\right), \\
& Z\left(u+2 \mathbb{K} \mid k^{2}\right)=Z\left(u \mid k^{2}\right) .
\end{aligned}
$$

Useful representations for $Z\left(u \mid k^{2}\right)$ are the integral representation

$Z\left(\operatorname{sn}^{-1}\left(y \mid k^{2}\right) \mid k^{2}\right)=\int_{0}^{y} \mathrm{~d} t\left[\sqrt{\frac{1-k^{2} t^{2}}{1-t^{2}}}-\frac{\mathbb{E}\left(k^{2}\right)}{\mathbb{K}\left(k^{2}\right)} \frac{1}{\sqrt{\left(1-t^{2}\right)\left(1-k^{2} t^{2}\right)}}\right]$

and

$$
\mathbb{Z}\left(\alpha ; k^{2}\right)=\int_{0}^{\alpha} \mathrm{d} u \mathrm{dn}^{2}\left(u ; k^{2}\right)-\frac{\mathbb{E}\left(k^{2}\right)}{\mathbb{K}\left(k^{2}\right)} \alpha .
$$

We also recall the following series representation:

$$
Z\left(u \mid k^{2}\right)=-\frac{\pi}{2 \mathbb{K}^{\prime} \mathbb{K}} u+\frac{\pi}{2 \mathbb{K}^{\prime}} \sum_{n=-\infty}^{\infty} \tanh \left(\frac{\pi}{2 \mathbb{K}^{\prime}}(u-2 n \mathbb{K})\right) .
$$




\section{A.4. Landen transformations useful for folded string fluctuation operators}

(a) Bosonic fluctuation $\phi$. Consider the bosonic fluctuation (3.28)-(3.30):

$$
\mathcal{O}_{\phi}=-\partial_{\sigma}^{2}+2 \omega^{2} k^{2} \operatorname{sn}^{2}\left(\omega \sigma+\mathbb{K} \mid k^{2}\right)+2 \omega^{2} \mathrm{~ns}^{2}\left(\omega \sigma+\mathbb{K} \mid k^{2}\right) .
$$

In the potential in $\mathcal{O}_{\phi}$ one can use

$$
\operatorname{sn}^{2}\left(u \mid k^{2}\right)=\frac{1-\operatorname{cn}\left(2 u \mid k^{2}\right)}{1+\operatorname{dn}\left(2 u \mid k^{2}\right)}, \quad \operatorname{ns}\left(u \mid k^{2}\right)=\operatorname{isn}\left(u+i \mathbb{K}^{\prime} \mid k^{2}\right)
$$

and perform the Landen transformation

$$
\operatorname{sn}\left(\left(1+\tilde{k}^{\prime}\right) u \mid k^{2}\right)=\left(1+\tilde{k}^{\prime}\right) \operatorname{sn}\left(u \mid \tilde{k}^{2}\right) \operatorname{cd}\left(u \mid \tilde{k}^{2}\right)
$$

with $\tilde{k}$ defined in (A.25). Rescaling the variable

$$
\bar{\sigma}=\frac{2 \omega \sigma}{1+\tilde{k}^{\prime}}
$$

one obtains the single-gap Lamé operator

$$
\mathcal{O}_{\phi}=-\partial_{z}^{2}+\tilde{k}^{2} \operatorname{sn}\left(z+\frac{i \tilde{\mathbb{K}}^{\prime}}{1+\tilde{k}^{\prime}} \mid \tilde{k}^{2}\right)-\tilde{k}^{2}+\frac{\pi^{2} \Omega^{2}}{4 \widetilde{\mathbb{K}}^{2}}
$$

with periodicity $\phi(z)=\phi(z+4 \widetilde{\mathbb{K}})$ in the rescaled variable $z=\frac{2 \widetilde{\mathbb{K}}}{\pi} \sigma$. Note that the imaginary shift of $x$ makes the potential singular, as it must be from the original form (3.30), where singularities are manifest at $\sigma=\left(n+\frac{1}{2}\right) \pi, n \in \mathbf{N}$. Such an imaginary part, however, does not affect the discussion leading to the determinant expression ${ }^{19}$.

(b) Fermionic fluctuations. Consider the fermionic fluctuations (3.28)-(3.31):

$$
\mathcal{O}_{\psi_{ \pm}}=-\frac{\mathrm{d}^{2}}{\mathrm{~d} \sigma^{2}}+\kappa^{2} \operatorname{sn}^{2}\left(\omega \sigma+\mathbb{K} \mid k^{2}\right) \pm \kappa \omega \operatorname{cn}\left(\omega \sigma+\mathbb{K} \mid k^{2}\right) \operatorname{dn}\left(\omega \sigma+\mathbb{K} \mid k^{2}\right)+\Omega^{2}
$$

One can use the Landen transformation

$$
\begin{aligned}
& \operatorname{sn}\left(\left(1+\tilde{k}^{\prime}\right) u \mid k^{2}\right)=\left(1+\tilde{k}^{\prime}\right) \operatorname{sn}\left(u \mid \tilde{k}^{2}\right) \operatorname{cd}\left(u \mid \tilde{k}^{2}\right), \\
& \operatorname{cn}\left(\left(1+\tilde{k}^{\prime}\right) u \mid k^{2}\right)=\operatorname{nd}\left(u \mid \tilde{k}^{2}\right)-\left(1+\tilde{k}^{\prime}\right) \operatorname{sn}\left(u \mid \tilde{k}^{2}\right) \operatorname{sd}\left(u \mid \tilde{k}^{2}\right), \\
& \operatorname{dn}\left(\left(1+\tilde{k}^{\prime}\right) u \mid k^{2}\right)=\operatorname{nd}\left(u \mid \tilde{k}^{2}\right)-\left(1-\tilde{k}^{\prime}\right) \operatorname{sn}\left(u \mid \tilde{k}^{2}\right) \operatorname{sd}\left(u \mid \tilde{k}^{2}\right),
\end{aligned}
$$

where

$$
k=\frac{1-\tilde{k}^{\prime}}{1+\tilde{k}^{\prime}} \quad \Leftrightarrow \quad \tilde{k}^{2}=\frac{4 k}{(1+k)^{2}}
$$

and the relations of the parameters with the new modulus $\tilde{k}$ are

$$
\kappa=\frac{1-\tilde{k}^{\prime}}{1+\tilde{k}^{\prime}} \omega \quad \Longrightarrow \quad \tilde{k}^{\prime}=\frac{\omega-\kappa}{\omega+\kappa}, \quad \tilde{k}^{2}=\frac{4 \kappa \omega}{(\omega+\kappa)^{2}} .
$$

Rescaling then the variable

$$
y=\frac{\omega \sigma}{1+\tilde{k}^{\prime}}=\frac{\tilde{\mathbb{K}} \sigma}{\pi}
$$

and exploiting the relations

$$
\mathbb{K}\left(k^{2}\right)=\frac{1+\tilde{k}^{\prime}}{2} \mathbb{K}\left(\tilde{k}^{2}\right), \quad \mathbb{E}\left(k^{2}\right)=\frac{1}{1+\tilde{k}^{\prime}}\left(\mathbb{E}\left(\tilde{k}^{2}\right)+\tilde{k}^{\prime} \mathbb{K}\left(\tilde{k}^{2}\right)\right)
$$

19 In particular, it does not affect the monodromy of the potential. 
and

$$
\frac{\mathbb{E}\left(k^{2}\right)}{\mathbb{K}\left(k^{2}\right)}=\frac{2}{\left(1+\tilde{k}^{\prime}\right)^{2}}\left[\frac{\mathbb{E}\left(\tilde{k}^{2}\right)}{\mathbb{K}\left(\tilde{k}^{2}\right)}+\tilde{k}^{\prime 2}\right],
$$

one obtains two single-gap Lamé operators

$$
\begin{aligned}
& \mathcal{O}_{\psi_{+}}=-\partial_{y}^{2}+2 \tilde{k}^{2} \operatorname{sn}^{2}\left(y+\frac{\widetilde{\mathbb{K}}}{2} \mid \tilde{k}^{2}\right)-\tilde{k}^{2}+\frac{\pi^{2} \Omega^{2}}{\widetilde{\mathbb{K}}^{2}} \\
& \left.\mathcal{O}_{\psi_{-}}=-\partial_{y}^{2}+2 \tilde{k}^{2} \operatorname{sn}^{2}\left(y+\frac{3 \widetilde{\mathbb{K}}}{2}\right) \mid \tilde{k}^{2}\right)-\tilde{k}^{2}+\frac{\pi^{2} \Omega^{2}}{\widetilde{\mathbb{K}}^{2}}
\end{aligned}
$$

where the new elliptic parameter is $\tilde{k}^{2}=\frac{4 k}{(1+k)^{2}}, \tilde{\mathbb{K}}=\mathbb{K}\left(\tilde{k}^{2}\right)$, and the periodicity $\psi_{ \pm}(y)=\psi_{ \pm}(y+2 \tilde{\mathbb{K}})$ is in the new variable $y=\frac{\widetilde{\mathbb{K}}}{\pi} \sigma$.

\section{Appendix B. Determinant via the Gel'fand-Yaglom method}

For a periodic potential, we can compute the determinant via the discriminant as in (4.7). In certain special cases, the discriminant can be found exactly because we know the explicit solutions $f_{1,2}$ in (4.2). This is the case, for example, for the constant potential $V(x)=m^{2}$, and also for the single-gap Lamé potential $V(x)=2 k^{2} \mathrm{sn}^{2}\left(x \mid k^{2}\right)$. But in our comparison between the static gauge and conformal gauge we will need the determinant for coupled operators, and here we can use the Gel'fand-Yaglom theorem. To introduce this, we first state it for uncoupled operators, and then for coupled operators.

For the uncoupled equation (4.1) with periodic or antiperiodic boundary conditions, the Gel'fand-Yaglom method [28, 35] is simply an evaluation of the discriminant. That is, we numerically (or analytically, in special cases) evaluate the discriminant, using initial value boundary conditions (4.2) for the two independent functions $f_{1,2}(x ; \Lambda)$. Note that here $\Lambda$ is just a parameter, so we solve a homogeneous problem, with initial value conditions, which is numerically trivial. In fact, we can specify the initial conditions at any arbitrary point $\bar{x}$, and simply evolve through one period to evaluate the discriminant. That is, we can take initial conditions

$$
\begin{array}{ll}
f_{1}(\bar{x} ; \Lambda)=1 ; & f_{1}^{\prime}(\bar{x} ; \Lambda)=0 \\
f_{2}(\bar{x} ; \Lambda)=0 ; & f_{2}^{\prime}(\bar{x} ; \Lambda)=1 .
\end{array}
$$

Then the Gelf' and-Yaglom theorem states that the determinant with period $L$ is

$$
\begin{aligned}
\operatorname{det}_{P}^{\mathrm{GY}}(\Lambda) & =\Delta(\Lambda)-2 \\
& =f_{1}(\bar{x}+L ; \Lambda)+f_{2}^{\prime}(\bar{x}+L ; \Lambda)-2 .
\end{aligned}
$$

We can illustrate this method for the single-gap Lamé system, by taking two linear combinations of $f_{+}$and $f_{-}$in (4.23):

$$
f_{1}(x)=m_{1} f_{+}(x)+m_{2} f_{-}(x), \quad f_{2}(x)=n_{1} f_{+}(x)+n_{2} f_{-}(x)
$$

such that the (B.1) are satisfied and $\alpha$ is given by (4.22). One finds

$m_{1}=-\frac{f_{-}^{\prime}(\bar{x})}{D(\bar{x})}, \quad m_{1}=\frac{f_{+}^{\prime}(\bar{x})}{D(\bar{x})} ; \quad n_{1}=\frac{f_{-}(\bar{x})}{D(\bar{x})}, \quad n_{2}=-\frac{f_{+}(\bar{x})}{D(\bar{x})}$

where

$$
D(\bar{x})=f_{+}^{\prime}(\bar{x}) f_{-}(\bar{x})-f_{-}^{\prime}(\bar{x}) f_{+}(\bar{x}) .
$$


Exploiting the monodromy

$f_{ \pm}(x+2 \mathbb{K})=-f_{ \pm}(x) \mathrm{e}^{\mp 2 \mathbb{K} Z\left(\alpha \mid k^{2}\right)}, \quad f_{ \pm}^{\prime}(x+2 \mathbb{K})=-f_{ \pm}^{\prime}(x) \mathrm{e}^{\mp 2 \mathbb{K} Z\left(\alpha \mid k^{2}\right)}$,

it is then easy to check that the expression for the determinant (B.2) yields

$$
\operatorname{det}_{\mathrm{P}}=2 \cosh \left[4 \mathbb{K} Z\left(\alpha \mid k^{2}\right)\right]-2=4 \sinh ^{2}\left[2 \mathbb{K} Z\left(\alpha \mid k^{2}\right)\right]
$$

as before (4.26). Note the important observation that this result is independent of the initial point $\bar{x}$. A specific example of the linear combinations (B.3) satisfying (B.1) at the initial point $\bar{x}=0$ are the solutions [29]

$f_{1}(x)=f_{+}(x)-f_{-}(x), \quad f_{2}(x)=\frac{\operatorname{sn}\left(\alpha \mid k^{2}\right)}{\operatorname{cn}\left(\alpha \mid k^{2}\right) \operatorname{dn}\left(\alpha \mid k^{2}\right)}\left(f_{+}(x)+f_{-}(x)\right)$.

For a system of coupled equations, the Gel'fand-Yaglom theorem generalizes in a straightforward manner. Consider (4.1) with $V(x)$ now an $n \times n$ matrix, and $f(x)$ an $n$-component column vector. Then we define $2 n$ independent solutions $f_{1}^{(a)}(x ; \Lambda)$ and $f_{2}^{(a)}(x ; \Lambda)$, for $a=1,2, \ldots, n$, with the initial conditions expressed as a $2 n \times 2 n$ matrix:

$$
\left(\begin{array}{llllll}
f_{1}^{(1)}(0 ; \Lambda) & \cdots & f_{1}^{(n)}(0 ; \Lambda) & f_{1}^{(1) \prime}(0 ; \Lambda) & \cdots & f_{1}^{(n) \prime}(0 ; \Lambda) \\
f_{2}^{(1)}(0 ; \Lambda) & \cdots & f_{2}^{(n)}(0 ; \Lambda) & f_{2}^{(1) \prime}(0 ; \Lambda) & \cdots & f_{2}^{(n) \prime}(0 ; \Lambda)
\end{array}\right)=\mathbb{I}_{2 n \times 2 n} .
$$

Then the Gel'fand-Yaglom theorem states that the infinite-dimensional determinant can be expressed as a finite dimensional determinant:

$$
\begin{aligned}
\operatorname{det}_{P}[ & \left.-\partial_{x}^{2}+V(x)-\Lambda\right]=-\operatorname{det}_{2 n \times 2 n} \\
& \times\left[\mathbb{I}-\left(\begin{array}{llllll}
f_{1}^{(1)}(L ; \Lambda) & \cdots & f_{1}^{(n)}(L ; \Lambda) & f_{1}^{(1) \prime}(L ; \Lambda) & \cdots & f_{1}^{(n) \prime}(L ; \Lambda) \\
f_{2}^{(1)}(L ; \Lambda) & \cdots & f_{2}^{(n)}(L ; \Lambda) & f_{2}^{(1) \prime}(L ; \Lambda) & \cdots & f_{2}^{(n) \prime}(L ; \Lambda)
\end{array}\right)\right] .
\end{aligned}
$$

Again, this is completely straightforward to evaluate numerically. This was used to evaluate the determinant of the coupled operators in the conformal-gauge example discussed in section 5 .

\section{Appendix C. Relevant expansions of elliptic and Jacobi functions}

\section{C.1. Expansions of $\mathbb{K}$ and $\mathbb{E}(k \rightarrow 1)$}

The expansion of the complete elliptic integrals in $k^{\prime 2}$ for $k \rightarrow 1$ reads (with $L=\ln 4 / k^{\prime}$ )

$$
\begin{aligned}
& \mathbb{K}\left(k^{2}\right)=L+\frac{1}{4}(L-1) k^{\prime 2}+\frac{9}{64}\left(L-\frac{7}{6}\right) k^{\prime 4}+\frac{25}{256}\left(L-\frac{37}{30}\right) k^{\prime 6}+\cdots \\
& \mathbb{E}\left(k^{2}\right)=1+\frac{1}{2}\left(L-\frac{1}{2}\right) k^{\prime 2}+\frac{3}{16}\left(L-\frac{13}{12}\right) k^{\prime 4}+\frac{15}{128}\left(L-\frac{6}{5}\right) k^{\prime 6}+\frac{175}{2048}\left(L-\frac{1051}{840}\right) k^{\prime 8}+\cdots
\end{aligned}
$$

Also

$$
\begin{aligned}
\frac{\mathbb{E}\left(k^{2}\right)}{\mathbb{K}\left(k^{2}\right)}=\frac{1}{L}+ & \left(\frac{1}{2}-\frac{1}{2 L}+\frac{1}{4 L^{2}}\right) k^{\prime 2}+\left(\frac{1}{16}-\frac{3}{32 L}-\frac{3}{128 L^{2}}+\frac{1}{16 L^{3}}\right) k^{\prime 4} \\
& +\left(\frac{1}{32}-\frac{3}{64 L}-\frac{11}{768 L^{2}}+\frac{5}{256 L^{3}}+\frac{1}{64 L^{4}}\right) k^{\prime 6}+\cdots
\end{aligned}
$$

This gives

$$
\kappa=\kappa_{0}-\frac{1}{4 \pi}\left(\pi \kappa_{0}-2\right) \eta+\frac{9}{64 \pi}\left(\pi \kappa_{0}-\frac{7}{3}\right) \eta^{2}-\frac{25}{256 \pi}\left(\pi \kappa_{0}-\frac{37}{15}\right) \eta^{3}+\cdots .
$$




\section{C.2. Expansion of the Jacobi zeta function $Z(k \rightarrow 1)$}

Consider the integral representation of the Jacobi zeta function (A.15):

$$
f(y)=Z\left(\operatorname{sn}^{-1}\left(y \mid k^{2}\right) \mid k^{2}\right) .
$$

We find for the asymptotics for $k \rightarrow 1$ (setting $L=\ln \frac{4}{k^{\prime}}$ )

$$
\begin{aligned}
f(y)=y-\frac{1}{L} & \int_{0}^{y} \frac{\mathrm{d} t}{1-t^{2}}-\frac{1}{2} k^{\prime 2} y+\frac{1}{2 L} k^{\prime 2} \int_{0}^{y} \frac{\mathrm{d} t}{\left(1-t^{2}\right)^{2}}-\frac{1}{4 L^{2}} k^{\prime 2} \int_{0}^{y} \frac{\mathrm{d} t}{1-t^{2}} \\
& +k^{\prime 4} \int_{0}^{y} \mathrm{~d} t\left[-\frac{1}{16} \frac{1-5 t^{2}+2 t^{4}}{\left(1-t^{2}\right)^{2}}-\frac{1}{32 L} \frac{-3+14 t^{2}+t^{4}}{\left(1-t^{2}\right)^{3}}\right. \\
& \left.+\frac{1}{128 L^{2}} \frac{3+13 t^{2}}{\left(1-t^{2}\right)^{2}}-\frac{1}{16 L^{3}} \frac{1}{1-t^{2}}\right],
\end{aligned}
$$

which gives

$$
\begin{aligned}
f(y)=y-\frac{1}{L} & \operatorname{artanh}(y)+\frac{1}{2} k^{\prime 2}\left[-y+\frac{1}{2 L}\left(\frac{y}{1-y^{2}}+\operatorname{artanh}(y)\right)-\frac{1}{2 L^{2}} \operatorname{artanh}(y)\right] \\
& +\frac{1}{16} k^{\prime 4}\left[-\frac{y\left(1-2 y^{2}\right)}{1-y^{2}}+\frac{1}{4 L}\left(\frac{y\left(1-7 y^{2}\right)}{\left(1-y^{2}\right)^{2}}+5 \operatorname{artanh}(y)\right)\right. \\
& \left.+\frac{1}{8 L^{2}}\left(\frac{8 y}{1-y^{2}}-5 \operatorname{artanh}(y)\right)-\frac{1}{L^{3}} \operatorname{artanh}(y)\right] \\
& +\frac{1}{32} k^{6}\left[-\frac{y\left(1-2 y^{2}+2 y^{4}\right)}{\left(1-y^{2}\right)^{2}}+\frac{1}{8 L}\left(\frac{y\left(3-20 y^{2}+57 y^{4}\right)}{3\left(1-y^{2}\right)^{3}}+11 \operatorname{artanh}(y)\right)\right. \\
& +\frac{1}{48 L^{2}}\left(\frac{9 y\left(5-9 y^{2}\right)}{\left(1-y^{2}\right)^{2}}-23 \operatorname{artanh}(y)\right) \\
& \left.-\frac{1}{8 L^{3}}\left(\frac{4 y}{1-y^{2}}-9 \operatorname{artanh}(y)\right)-\frac{1}{2 L^{4}} \operatorname{artanh}(y)\right]+\cdots
\end{aligned}
$$

Using the above results, one can read off the expansions of the relevant quantities appearing in the static-gauge fluctuation determinants:

$$
\begin{aligned}
2 \mathbb{K}\left(k^{2}\right) Z\left(\alpha_{\beta} \mid k^{2}\right) & \sim \kappa_{0} \pi x-2 \tanh ^{-1} x+\eta \frac{\left(\kappa_{0} \pi x^{2}-x^{2}-\kappa_{0} \pi+2\right)}{\kappa_{0} \pi x\left(x^{2}-1\right)}+\frac{\eta^{2}}{32 \kappa_{0}^{2} \pi^{2} x^{3}\left(x^{2}-1\right)^{2}} \\
& \times\left[\kappa_{0}^{3} \pi^{3} x^{6}-13 \kappa_{0}^{2} \pi^{2} x^{6}+21 \kappa_{0} \pi x^{6}-4 \kappa_{0}^{3} \pi^{3} x^{4}+30 \kappa_{0}^{2} \pi^{2} x^{4}-71 \kappa_{0} \pi x^{4}\right. \\
+ & \left.32 x^{4}+3 \kappa_{0}^{3} \pi^{3} x^{2}-19 \kappa_{0}^{2} \pi^{2} x^{2}+66 \kappa_{0} \pi x^{2}-80 x^{2}-16 \kappa_{0} \pi+32\right]+\cdots
\end{aligned}
$$

$$
\begin{aligned}
2 \mathbb{K}\left(\tilde{k}^{2}\right) Z\left(\alpha_{\phi} \mid \tilde{k^{2}}\right) & \sim \kappa_{0} \pi y-2 \tanh ^{-1} \frac{y}{2}+\eta \frac{2\left(\kappa_{0} \pi-1\right)}{\kappa_{0} \pi y}+\frac{\eta^{2}}{16 \pi^{2} y^{3} \omega^{2}} \\
& \times\left(\kappa_{0}^{3} \pi^{3} y^{4}-13 \kappa_{0}^{2} \pi^{2} y^{4}+21 \kappa_{0} \pi y^{4}-4 \kappa_{0}^{3} \pi^{3} y^{2}+56 \kappa_{0}^{2} \pi^{2} y^{2}\right. \\
& \left.-116 \kappa_{0} \pi y^{2}+32 y^{2}+128 \kappa_{0} \pi-128\right)+\cdots
\end{aligned}
$$

$\mathbb{K}(\bar{v}) Z(\tilde{\alpha}) \sim \kappa_{0} \pi z-\tanh ^{-1} z+\eta \frac{\left(\kappa_{0} \pi-1\right)}{2 \kappa_{0} \pi z}+\frac{\eta^{2}}{64 \kappa_{0}^{2} \pi^{2} z^{3}\left(z^{2}-1\right)}\left(\kappa_{0}^{3} \pi^{3} z^{4}-13 \kappa_{0}^{2} \pi^{2} z^{4}\right.$

$$
\left.+21 \kappa_{0} \pi z^{4}-\kappa_{0}^{3} \pi^{3} z^{2}+14 \kappa_{0}^{2} \pi^{2} z^{2}-29 \kappa_{0} \pi z^{2}+8 z^{2}+8 \kappa_{0} \pi-8\right)+\cdots
$$

where $x, y, z$ are defined in (6.5)-(6.7). 


\section{C.3. Expansions of $\mathbb{K}$ and $\mathbb{E}(k \rightarrow 0)$}

The first few orders of the $\kappa \rightarrow 0$ expansions for the elliptic integrals $\mathbb{K}$ and $\mathcal{E}$ read

$$
\begin{aligned}
& \mathbb{K}\left(k^{2}\right)=\frac{\pi}{2}+\frac{\pi}{8} k^{2}+\frac{9 \pi}{128} k^{4}+\frac{25 \pi}{512} k^{6}+\frac{1225 \pi}{32768} k^{8}+O\left(k^{9}\right) \\
& \mathbb{E}\left(k^{2}\right)=\frac{\pi}{2}-\frac{\pi}{8} k^{2}-\frac{3 \pi}{128} k^{4}-\frac{5 \pi}{512} k^{6}-\frac{175 \pi}{32768} k^{8}+O\left(k^{9}\right) .
\end{aligned}
$$

\section{C.4. Expansion of the Jacobi zeta function $Z(k \rightarrow 0)$}

As an efficient way to evaluate the small-spin expansion $(k \rightarrow 0, \eta \rightarrow \infty)$ presented in section 7 is to first compute the expansion of $\partial Z\left(\alpha \mid k^{2}\right) / \partial \Omega$, where the dependence of $Z$ on $\Omega$ is via $\alpha$ as the solution of (5.2)-(5.4), and then perform an indefinite integration over $\Omega$.

Using (A.16) valid for $0<\alpha<\mathbb{K}$, after some straightforward manipulations one can write

$\frac{\partial Z\left(\alpha \mid k^{2}\right)}{\partial \Omega}=\frac{1}{\sqrt{1-\operatorname{sn}^{2}\left(\alpha \mid k^{2}\right)} \sqrt{1-k^{2} \mathrm{sn}^{2}\left(\alpha \mid k^{2}\right)}} \frac{\partial \operatorname{sn}\left(\alpha \mid k^{2}\right)}{\partial \Omega}\left[1-k^{2} \operatorname{sn}\left(\alpha \mid k^{2}\right)-\frac{\mathbb{E}}{\mathbb{K}}\right]$.

Considering the determinant for beta modes

$\operatorname{det} \mathcal{O}_{\beta}=4 \sinh ^{2}\left[2 \mathbb{K} Z\left(\alpha \mid k^{2}\right)\right], \quad \operatorname{sn}\left(\alpha \mid k^{2}\right)=\frac{1}{k} \sqrt{1+k^{2}+\left(\frac{\pi \Omega}{2 \mathbb{K}}\right)^{2}}$

one can see that $\operatorname{sn}\left(\alpha \mid k^{2}\right)>1$. So before proceeding with the short-string expansion, one needs the following transformation:

$$
\alpha=\beta+\mathbb{K}+\mathrm{i} \mathbb{K}^{\prime}
$$

which gives

$$
\operatorname{sn}\left(\beta \mid k^{2}\right)=\sqrt{\frac{k^{2}+\left(\frac{\pi \Omega}{2 \mathbb{K}}\right)^{2}}{1+\left(\frac{\pi \Omega}{2 \mathbb{K}}\right)^{2}}}<1
$$

This affects the determinant in the following way:

$$
\operatorname{det} \mathcal{O}_{\beta}=4 \sinh ^{2}\left[2 \mathbb{K} Z\left(\beta \mid k^{2}\right)-2 \mathbb{K} \frac{\operatorname{sn}\left(\beta \mid k^{2}\right) \operatorname{dn}\left(\beta \mid k^{2}\right)}{\operatorname{cn}\left(\beta \mid k^{2}\right)}-\mathrm{i} \pi\right]
$$

Substituting the explicit expression for $\operatorname{sn}\left(\beta \mid k^{2}\right)$ given by (C.16) into (C.13), expanding then for large $\eta$ and integrating back in $\Omega$, one can easily evaluate the expansions for the relevant determinants. In the case of the fluctuation $\beta$ it is finally

$$
\begin{aligned}
\operatorname{det} \mathcal{O}_{\beta} \equiv 4 \sinh ^{2}\left[2 \mathbb{K} Z\left(\alpha_{\beta} \mid k^{2}\right)\right]=4 \sinh ^{2}(\pi \Omega)+\frac{1}{\eta} \frac{2 \pi \sinh (2 \pi \Omega)}{\Omega} \\
+\frac{1}{\eta^{2}}\left[\frac{\pi^{2} \cosh (2 \pi \Omega)}{\Omega^{2}}-\frac{\pi\left(3 \Omega^{4}+6 \Omega^{2}+2\right) \sinh (2 \pi \Omega)}{4\left(\Omega^{5}+\Omega^{3}\right)}\right]+O\left(\frac{1}{\eta^{3}}\right) .
\end{aligned}
$$

Applying this approach to the fermion determinant

$$
\operatorname{det} \mathcal{O}_{\psi}=-4 \cosh ^{2}\left[\tilde{\mathbb{K}} Z\left(\alpha \mid \tilde{k}^{2}\right)\right], \quad \operatorname{sn}\left(\alpha \mid \tilde{k}^{2}\right)=\frac{1}{\tilde{k}} \sqrt{1+\left(\frac{\pi \Omega}{\tilde{\mathbb{K}}}\right)^{2}}
$$


by using $\alpha=\beta+\tilde{\mathbb{K}}+\mathrm{i} \tilde{\mathbb{K}}^{\prime}$ with

$$
\operatorname{sn}\left(\beta \mid \tilde{k}^{2}\right)=\sqrt{\frac{\left(\frac{\pi \Omega}{\tilde{\mathbb{K}}}\right)^{2}}{1-\tilde{k}^{2}+\left(\frac{\pi \Omega}{\tilde{\mathbb{R}}}\right)^{2}}}
$$

gives

$$
\begin{aligned}
\operatorname{det} \mathcal{O}_{\psi} & =-4 \cosh ^{2}\left[\tilde{\mathbb{K}} Z\left(\beta \mid \tilde{k}^{2}\right)-\tilde{\mathbb{K}} \frac{\operatorname{sn}\left(\beta \mid \tilde{k}^{2}\right) \operatorname{dn}\left(\beta \mid \tilde{k}^{2}\right)}{\operatorname{cn}\left(\beta \mid \tilde{k}^{2}\right)}-\frac{\mathrm{i} \pi}{2}\right] \\
& =4 \sinh ^{2}\left[\tilde{\mathbb{K}} Z\left(\beta \mid \tilde{k}^{2}\right)-\tilde{\mathbb{K}} \frac{\operatorname{sn}\left(\beta \mid \tilde{k}^{2}\right) \operatorname{dn}\left(\beta \mid \tilde{k}^{2}\right)}{\operatorname{cn}\left(\beta \mid \tilde{k}^{2}\right)}\right],
\end{aligned}
$$

where the term $\mathrm{i} \pi / 2$ flips the cosh to sinh. The expansion of this expression in $1 / \eta$ gives

$$
\operatorname{det} \mathcal{O}_{\psi}(\Omega)=D_{\psi}^{(0)}(\Omega)+\frac{1}{\eta} D_{\psi}^{(1)}(\Omega)+\frac{1}{\eta^{2}} D_{\psi}^{(2)}(\Omega)+\cdots
$$

with

$D_{\psi}^{(0)}(\Omega)=4 \sinh (\pi \Omega)$,

$D_{\psi}^{(1)}(\Omega)=\frac{4 \pi \Omega \sinh (2 \pi \Omega)}{1+4 \Omega^{2}}$,

$D_{\psi}^{(2)}(\Omega)=\frac{4 \pi^{2} \Omega^{2} \cosh (2 \pi \Omega)}{\left(1+4 \Omega^{2}\right)^{2}}-\frac{\pi \Omega\left(48 \Omega^{2}\left(1+\Omega^{2}\right)+1\right) \sinh (2 \pi \Omega)}{2\left(1+4 \Omega^{2}\right)^{3}}$.

\section{Appendix D. Exponentially suppressed contributions}

As explained below (6.24) and around (6.35), in performing the large-spin expansion on the exact determinants we systematically adopted an approximation based on the replacement $\tanh (\cdots) \rightarrow 1$. The neglected terms are exponential in the large quantity $\kappa_{0}$ and give back powers of $\eta$, see (6.35). Lacking a better complete control of this approximation, we present in this appendix the evaluation of the leading large $\kappa_{0}$ correction to the one-loop energy due to the above replacement. It is clear that such leading correction comes indeed from the fermion determinant that has the following leading order in the formal small $\eta$, i.e. large spin, expansion:

$$
\mathcal{D}_{\psi, \mathrm{LO}}=\frac{1}{1-z^{2}}\left[1-z \tanh \left(\pi \kappa_{0} z\right)\right]^{2}, \quad z=\sqrt{1+\frac{\Omega^{2}}{\kappa_{0}^{2}}},
$$

and which we treated in the following approximated way:

$$
\mathcal{D}_{\psi, \mathrm{LO}}^{\text {approx }}=\frac{1}{1-z^{2}}(1-z)^{2}=\frac{1-z}{1+z} .
$$

The effect of this approximation in the one-loop energy is

$\Delta E_{1}=-\frac{1}{4 \pi \kappa} \cdot 8 \cdot \int_{-\infty}^{\infty} \mathrm{d} \Omega\left(\ln \mathcal{D}_{\psi, \mathrm{LO}}-\ln \mathcal{D}_{\psi, \mathrm{LO}}^{\text {approx }}\right)=-\frac{4 \kappa_{0}}{\pi \kappa} \int_{0}^{\infty} \mathrm{d} \bar{\Omega} F\left(\bar{\Omega} ; \kappa_{0}\right)$,

where

$$
F\left(\bar{\Omega} ; \kappa_{0}\right)=\ln \left(\frac{1-\sqrt{1+\bar{\Omega}^{2}} \tanh \left(\pi \kappa_{0} \sqrt{1+\bar{\Omega}^{2}}\right)}{1-\sqrt{1+\bar{\Omega}^{2}}}\right)^{2} .
$$




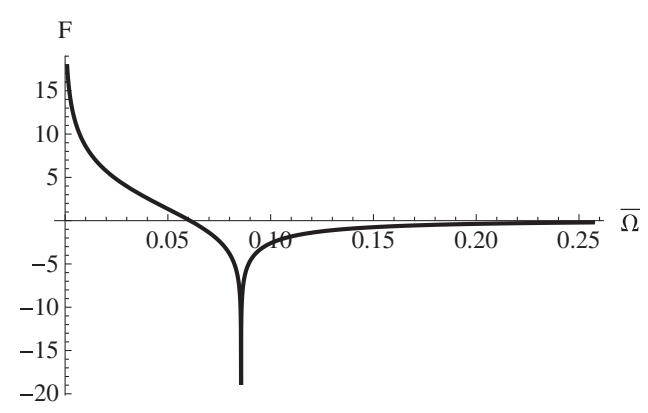

Figure D1. Shape of the function $F\left(\bar{\Omega} ; \kappa_{0}\right)$ at $\kappa_{0}=1$.

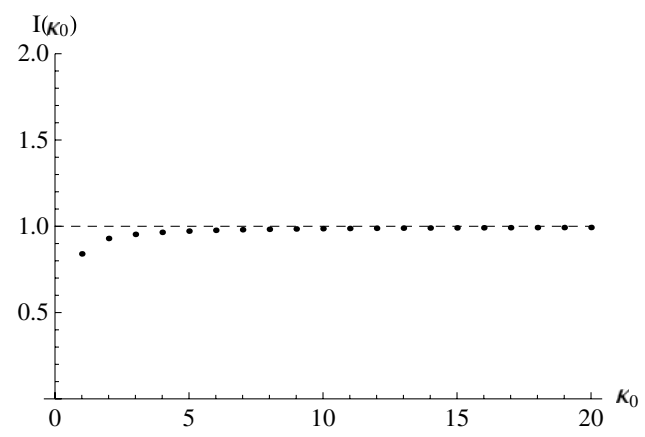

Figure D2. Numerical evaluation of the integral $I\left(\kappa_{0}\right)=\frac{1}{8 \pi \sqrt{\kappa_{0}}} \mathrm{e}^{2 \pi \kappa_{0}} \int_{0}^{\infty} \mathrm{d} \bar{\Omega} F\left(\bar{\Omega} ; \kappa_{0}\right)$ and comparison with the leading analytic prediction 1 (dashed line).

The function $F\left(\bar{\Omega} ; \kappa_{0}\right)$ has the generic shape shown in figure D1. The point where it goes to $-\infty$ is where the numerator inside the logarithm vanishes. This happens at approximately

$$
\bar{\Omega}^{*} \simeq 2 \mathrm{e}^{-\pi \kappa_{0}} .
$$

The large $\kappa_{0}$ analysis must be done carefully since the two regions $\bar{\Omega}<\bar{\Omega}^{*}$ and $\bar{\Omega}>\bar{\Omega}^{*}$ contribute the above integrals with opposite signs and large cancellations.

The final result is the estimate

$$
\int_{0}^{\infty} \mathrm{d} \bar{\Omega} F\left(\bar{\Omega} ; \kappa_{0}\right)=8 \pi \sqrt{\kappa_{0}} \mathrm{e}^{-2 \pi \kappa_{0}}+\cdots,
$$

whose accuracy is shown in figure D2. In terms of the one-loop energy, it is

$$
\Delta E_{1}=-32 \frac{\kappa_{0}^{3 / 2}}{\kappa} \mathrm{e}^{-2 \pi \kappa_{0}}+\cdots=\mathcal{O}\left(\eta^{2} \ln ^{1 / 2} \eta\right) .
$$

The peculiar half-integer exponent of $\ln \eta$ suggest that a systematic resummation of these corrections is needed possibly taking into account mixing with similar terms coming from the expansion of Bessel functions $K_{1}$ in equations (6.12)-(6.15). We did not evaluate the next correction, but figure D3 suggests that

$$
\int_{0}^{\infty} \mathrm{d} \bar{\Omega} F\left(\bar{\Omega} ; \kappa_{0}\right)=\left(8 \pi \sqrt{\kappa_{0}}+\frac{a}{{\sqrt{\kappa_{0}}}}+\cdots\right) \mathrm{e}^{-2 \pi \kappa_{0}}+\cdots,
$$

for some constant $a$. This term has the same form as the Bessel $K_{1}$ corrections. 


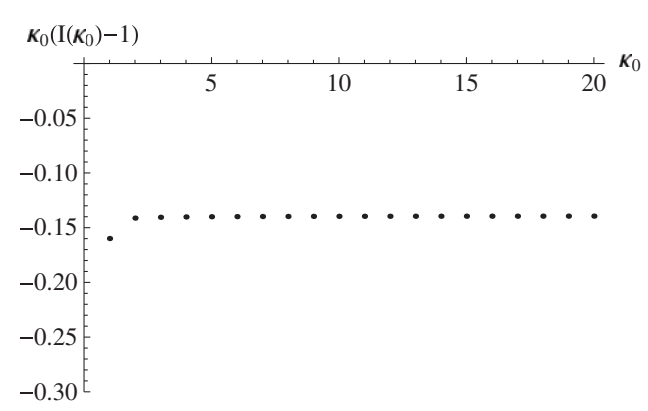

Figure D3. Numerical evaluation of the quantity $\kappa_{0}\left(I\left(\kappa_{0}\right)-1\right)$.

\section{Appendix E. Large-spin limit with a different approximation}

The analysis of the large-spin limit in section 6, leading to the large-spin limit expansion (6.36), agrees extremely well with the exact numerical dependence of the one-loop energy on the elliptic parameter $k$, as can be seen from figure 7. However, this expansion neglected exponential terms in the $k \rightarrow 1$ limit, as explained in section 6.1, and the significance of these neglected terms at smaller values of $k$ is not clear. In this section we consider another type of approximation that leads to a different type of large-spin expansion of the one-loop energy.

Start from the effective action

$$
\Gamma=-\frac{\mathcal{T}}{4 \pi} \int_{\mathbb{R}} \mathrm{d} \Omega \log \frac{\operatorname{det}^{8} \mathcal{O}_{\psi}}{{\operatorname{det} \mathcal{O}_{\phi}} \operatorname{det}^{2} \mathcal{O}_{\beta} \operatorname{det}^{5} \mathcal{O}_{0}},
$$

and integrate by parts (we also set $\mathcal{T}=1$ )

$$
\begin{aligned}
\Gamma & =\frac{1}{4 \pi} \int_{\mathbb{R}} \mathrm{d} \Omega \Omega \partial_{\Omega}\left[8 \log \operatorname{det} \mathcal{O}_{\psi}-\log \operatorname{det} \mathcal{O}_{\phi}-2 \log \operatorname{det} \mathcal{O}_{\beta}-5 \log \operatorname{det} \mathcal{O}_{0}\right] \\
& \equiv 8 \Gamma_{\psi}-\Gamma_{\phi}-2 \Gamma_{\beta}-5 \Gamma_{0} .
\end{aligned}
$$

We now systematically apply the approximate substitutions

$$
\log \left(4 \sinh ^{2} x\right) \rightarrow 2|x|, \quad \log \left(4 \cosh ^{2} x\right) \rightarrow 2 x,
$$

which also correspond to neglecting exponential terms, but now the approximation is made before integrating over $\Omega$. Then, the effective action can be computed exactly, i.e. without any leftover integral. As an example, let us consider the contribution from the $\phi$ mode. We convert the integral over $\Omega$ into an integral over the spectral parameter $\alpha_{\phi}$ as follows:

$$
\begin{aligned}
\Gamma_{\phi} & =\frac{1}{4 \pi} \int_{-\infty}^{\infty} \mathrm{d} \Omega \Omega \partial_{\Omega} \log \left(4 \sinh ^{2}\left(2 \widetilde{\mathbb{K}} Z\left(\alpha_{\phi} \mid \widetilde{k}^{2}\right)\right)\right. \\
& \left.\simeq \frac{2 \widetilde{\mathbb{K}}}{\pi} \int_{0}^{\infty} \mathrm{d} \Omega \Omega \partial_{\Omega} Z\left(\alpha_{\phi} \mid \widetilde{k}^{2}\right)\right) \\
& =\frac{2 \widetilde{\mathbb{K}}}{\pi} \int_{\alpha_{0}}^{i \widetilde{\mathbb{K}}^{\prime}} \mathrm{d} \alpha \Omega \partial_{\alpha} Z\left(\alpha_{\phi} \mid \widetilde{k}^{2}\right) \\
& =\left(\frac{2 \widetilde{\mathbb{K}}}{\pi}\right)^{2} \int_{\alpha_{0}}^{i \widetilde{\mathbb{K}}^{\prime}} \mathrm{d} \alpha \widetilde{\Omega}\left[\mathrm{dn}^{2}\left(\alpha \mid \widetilde{k}^{2}\right)-\frac{\widetilde{\mathbb{E}}}{\widetilde{\mathbb{K}}}\right],
\end{aligned}
$$

where

$$
\widetilde{\Omega}=\sqrt{-\operatorname{dn}^{2}\left(\alpha \mid \tilde{k}^{2}\right)}
$$


and $\alpha_{0}$ is the value associated with $\Omega=0$. The spectral parameter $\alpha_{\phi}$ takes values along the straight line joining $\widetilde{\mathbb{K}}+\mathrm{i} \widetilde{\mathbb{K}}^{\prime}$ to $i \widetilde{\mathbb{K}}^{\prime}$, so it is convenient to write

$$
\alpha=\widetilde{\mathbb{K}}+\mathrm{i} \widetilde{\mathbb{K}}^{\prime}-\beta
$$

and use the identity

$$
\operatorname{dn}^{2}\left(\widetilde{\mathbb{K}}+\mathrm{i} \widetilde{\mathbb{K}}^{\prime}-\beta \mid \widetilde{k}^{2}\right)=\left(\widetilde{k}^{2}-1\right) \operatorname{sc}^{2}\left(\beta \mid \widetilde{k}^{2}\right)
$$

We find

$$
\Gamma_{\phi} \simeq \widetilde{k}^{\prime}\left(\frac{2 \widetilde{\mathbb{K}}}{\pi}\right)^{2} \int_{0}^{\widetilde{\mathbb{K}}} \mathrm{d} \beta \operatorname{sc}\left(\beta \mid \widetilde{k}^{2}\right)\left[\frac{\widetilde{\mathbb{E}}}{\widetilde{\mathbb{K}}}+\widetilde{k}^{2 \prime} \operatorname{sc}^{2}\left(\beta \mid \widetilde{k}^{2}\right)\right] .
$$

A similar treatment leads to

$$
\Gamma_{\psi} \simeq \frac{1}{4} \Gamma_{\phi}
$$

within this approximation, and the following contribution from the $\beta$ modes:

$\Gamma_{\beta} \simeq\left(\frac{2 \mathbb{K}}{\pi}\right)^{2} \int_{\mathrm{sn}^{-1}\left(k \mid k^{2}\right)}^{\mathbb{K}} \mathrm{d} \beta \sqrt{\left(1-k^{2}\right) \mathrm{nc}^{2}\left(\beta \mid k^{2}\right)-1}\left[\frac{\mathbb{E}}{\mathbb{K}}+\left(1-k^{2}\right) \mathrm{sc}^{2}\left(\beta \mid k^{2}\right)\right]$.

It is convenient to introduce the variables

$$
s_{\phi}=\operatorname{sn}\left(\beta \mid \tilde{k}^{2}\right), \quad s_{\beta}=\operatorname{sn}\left(\beta \mid k^{2}\right) .
$$

The integrals can be computed in closed form, and after a long calculation, one finds

$$
\begin{aligned}
& \Gamma_{\phi}=-\frac{(k-1)^{2}}{\pi^{2}} \mathbb{K}^{2} \frac{1}{s_{\phi}-1}+\frac{4(\mathbb{K}-\mathbb{E}) \mathbb{K}}{\pi^{2}} \log \left(1-s_{\phi}\right)+\Gamma_{\phi}^{\text {finite }}, \\
& \Gamma_{\beta}=\frac{k^{2}-1}{\pi^{2}} \mathbb{K}^{2} \frac{1}{s_{\beta}-1}+\frac{2(\mathbb{K}-\mathbb{E}) \mathbb{K}}{\pi^{2}} \log \left(1-s_{\beta}\right)+\Gamma_{\beta}^{\text {finite }}
\end{aligned}
$$

The integrals are divergent at $s_{\phi}, s_{\beta} \rightarrow 1$, which is simply the individual UV divergence. Introducing a cut-off $\Omega_{\max }$ with

$$
\begin{aligned}
& s_{\beta, \max }=1-\frac{2 \mathbb{K}^{2}\left(k^{2}\right)}{\pi^{2} \Omega_{\max }^{2}}\left(1-k^{2}\right)+\cdots, \\
& s_{\phi, \max }=1-\frac{2 \widetilde{\mathbb{K}}^{2}\left(\widetilde{k}^{2}\right)}{\pi^{2} \Omega_{\max }^{2}}\left(1-\widetilde{k}^{2}\right)+\cdots, \\
& s_{\psi, \max }=1-\frac{\widetilde{\mathbb{K}}^{2}\left(k^{2}\right)}{2 \pi^{2} \Omega_{\max }^{2}}\left(1-\widetilde{k}^{2}\right)+\cdots,
\end{aligned}
$$

one checks that the pole cancels against the free field contribution which is

$$
\begin{aligned}
\Gamma_{0} & =\frac{1}{4 \pi} \int_{-\Omega_{\max }}^{\Omega_{\max }} \mathrm{d} \Omega \Omega \partial_{\Omega} \log \left(4 \sinh ^{2}(\pi \Omega)\right) \\
& \simeq \int_{0}^{\Omega_{\max }} \mathrm{d} \Omega \Omega=\frac{1}{2} \Omega_{\max }^{2}
\end{aligned}
$$




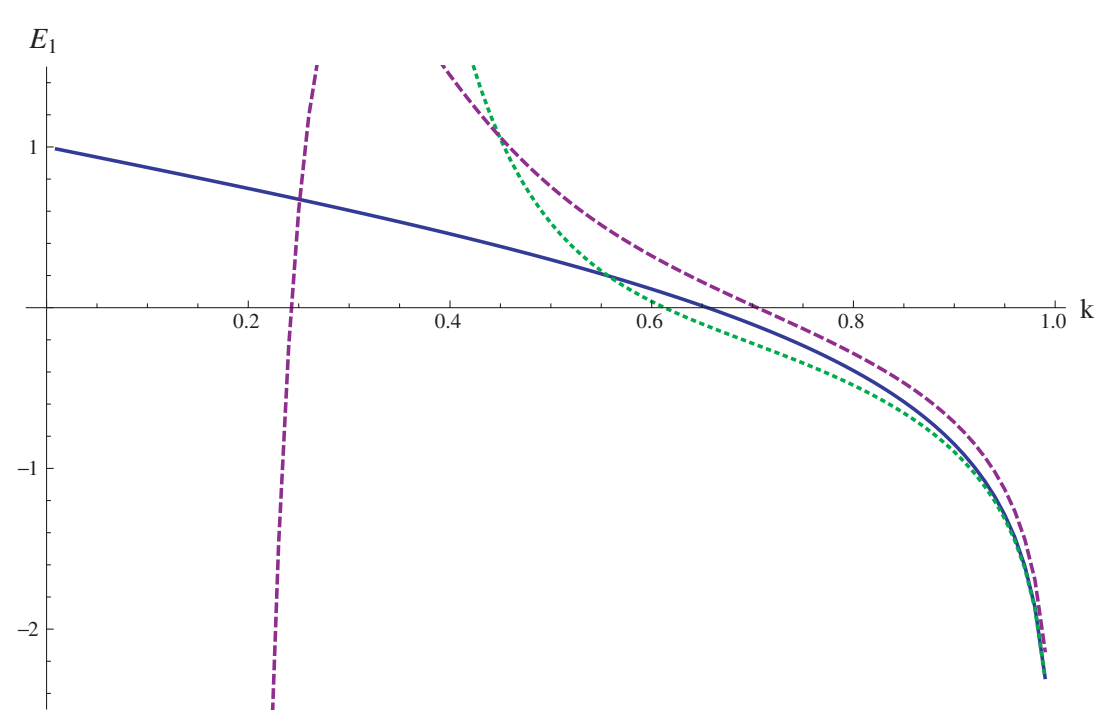

Figure E1. Plots of $E_{1}$ as a function of $k$ : the blue (solid) curve is found numerically from the exact expression (5.18) for generic values of $k$; the green (dotted) curve is found from an analytic expansion in the $k \rightarrow 1$ or large-spin limit, using the first two terms in (6.36); the purple (dashed) curve is the first two terms in the alternative $k \rightarrow 1$ expansion (E.19).

The logarithmic divergence cancels in the sum of the various mode contributions. The full result is quite compact and reads

$$
\begin{aligned}
\Gamma \simeq \frac{\mathbb{K}\left(k^{2}\right)}{\pi^{2} \mathbb{K}\left(k^{4}\right)} & {\left[4 \mathbb{K}\left(k^{2}\right) \mathbb{E}\left(k^{4}\right) \operatorname{Im} \mathbb{F}\left(\arcsin \frac{1}{k} \mid k^{4}\right)\right.} \\
& +\mathbb{K}\left(k^{2}\right)\left(\pi+2 \mathbb{K}\left(k^{4}\right)\left(k^{2}-2 \ln k-1-6 \ln 2\right)\right) \\
& \left.+4 \mathbb{K}\left(k^{4}\right) \mathbb{E}\left(k^{2}\right)\left(\ln (8 k)-\left(1+k^{2}\right) \operatorname{Im} \mathbb{F}\left(\arcsin \frac{1}{k} \mid k^{4}\right)\right)\right] .
\end{aligned}
$$

Since this compact expression is written in closed form in terms of elliptic functions, its $k \rightarrow 1$ expansion can be computed and the result to order $\mathcal{O}\left(\eta^{4}\right)$ is

$$
\begin{aligned}
\Gamma \simeq & \kappa_{0}\left(1+\frac{6 \log 2}{\pi}\right)-3 \kappa_{0}^{2} \log 2+\left[\kappa_{0}\left(-\frac{1}{\pi}-\frac{3 \log 2}{\pi}\right)+\frac{1}{2 \pi}+\frac{3 \log 2}{\pi^{2}}\right] \eta \\
+ & {\left[\kappa_{0}^{2}\left(-\frac{\pi}{32}-\frac{3 \log 2}{32}\right)+\kappa_{0}\left(\frac{1}{16}+\frac{1}{2 \pi}+\frac{39 \log 2}{32 \pi}\right)-\frac{13}{64 \pi}-\frac{1}{2 \pi^{2}}-\frac{63 \log 2}{32 \pi^{2}}\right] \eta^{2} } \\
+ & {\left[\kappa_{0}^{2}\left(\frac{1}{96}+\frac{\pi}{32}+\frac{3 \log 2}{32}\right)+\kappa_{0}\left(-\frac{3}{32}-\frac{1}{3 \pi}-\frac{13 \log 2}{16 \pi}\right)+\frac{29}{192 \pi}+\frac{29}{64 \pi^{2}}+\frac{85 \log 2}{64 \pi^{2}}\right] \eta^{3} } \\
+ & {\left[\kappa_{0}^{2}\left(-\frac{1}{64}-\frac{115 \pi}{4096}-\frac{693 \log 2}{8192}\right)+\kappa_{0}\left(\frac{51}{512}+\frac{25}{96 \pi}+\frac{10263 \log 2}{16384 \pi}\right)\right.} \\
& \left.-\frac{4397}{32768 \pi}-\frac{149}{384 \pi^{2}}-\frac{16403 \log 2}{16384 \pi^{2}}\right] \eta^{4}+\cdots .
\end{aligned}
$$

Recalling that $E_{1}=\Gamma / \kappa$, we see that this expansion is very similar to, but not precisely the same as, the $k \rightarrow 1$ expansion found in (6.36). A comparison of these two different approximations is presented in figure E1. Both agree with the exact result at large spin, but 
we see that the expansion in (6.36) provides a better approximation as $k \rightarrow 1$. Nevertheless, the approximation considered in this appendix may be of interest as it provides a closed-form expression.

\section{Appendix F. Static-gauge determinants in perturbation theory}

We repeat here an evaluation of the determinants in the short-string limit adopting the standard perturbation theory method of [9].

The basic idea is to compute

$$
\begin{gathered}
\ln \operatorname{det}\left(-\partial^{2}+\Omega^{2}+V\right)=\ln \operatorname{det}\left(-\partial^{2}+\Omega^{2}\right)+\operatorname{Tr}\left(\frac{1}{-\partial^{2}+\Omega^{2}} V\right) \\
-\frac{1}{2} \operatorname{Tr}\left(\frac{1}{-\partial^{2}+\Omega^{2}} V \frac{1}{-\partial^{2}+\Omega^{2}} V\right)+\cdots,
\end{gathered}
$$

evaluating the traces on the following basis: $|n\rangle=\frac{1}{\sqrt{2 \pi}} \mathrm{e}^{\mathrm{i} n \sigma}, n \in \mathbb{Z}, \sigma \in[0,2 \pi]$. In all cases, we define

$$
V_{n, m}=\frac{1}{2 \pi} \int_{0}^{2 \pi} \mathrm{d} \sigma \mathrm{e}^{\mathrm{i}(m-n) \sigma} V(\sigma)
$$

and also

$$
\ln \operatorname{det} \mathcal{O}_{f}=\ln \operatorname{det}\left(-\partial^{2}+\Omega^{2}\right)+X_{f}, \quad f=(\beta, \phi, \psi) .
$$

For the $\beta$ mode, we have (see (3.33) and the rescaling (3.35))

$$
V_{\beta}=\left(\frac{2 \mathbb{K}\left(k^{2}\right)}{\pi}\right)^{2} 2 k^{2} \operatorname{sn}^{2}\left(\frac{2 \mathbb{K}\left(k^{2}\right)}{\pi} \sigma \mid k^{2}\right)=\frac{2}{\eta} \sin ^{2} \sigma+\cdots,
$$

and the non-zero matrix elements

$$
V_{n, n}=\frac{1}{\eta}, \quad V_{n, n \pm 2}=-\frac{1}{2 \eta}
$$

Thus

$$
X_{\beta}=\frac{1}{\eta} \sum_{n \in \mathbb{Z}} \frac{1}{n^{2}+\Omega^{2}}
$$

For the $\phi$ mode, we have (see (A.21) and the rescaling $(3.36)^{20}$ )

$V_{\phi}=\left(\frac{2 \mathbb{K}\left(\tilde{k}^{2}\right)}{\pi}\right)^{2}\left[2 k^{2} \operatorname{sn}^{2}\left(\frac{2 \mathbb{K}\left(\tilde{k}^{2}\right)}{\pi} \sigma \mid \tilde{k}^{2}\right)-\tilde{k}^{2}\right]=-\frac{4}{\sqrt{\eta}} \cos (2 \sigma)+\frac{4}{\eta} \sin ^{2}(2 \sigma)+\cdots$,

and the non-zero matrix elements

$$
V_{n, n}=\frac{2}{\eta}, \quad V_{n, n \pm 2}=\frac{2}{\sqrt{\eta}}, \quad V_{n, n \pm 4}=-\frac{1}{\eta} .
$$

Thus

$$
X_{\phi}=\frac{2}{\eta} \sum_{n \in \mathbb{Z}} \frac{1}{n^{2}+\Omega^{2}}\left(1-\frac{1}{(n+2)^{2}+\Omega^{2}}-\frac{1}{(n-2)^{2}+\Omega^{2}}\right)
$$

\footnotetext{
20 The imaginary shift in the argument of the elliptic sine in (A.21) is irrelevant for the determinant calculation since it does not change the monodromy. For the check proposed here it is useful to consider the analytically continued potential (F.7).
}

40 
For the $\psi$ mode (see (A.30)-(A.31) and (3.37)), we have

$$
V_{\psi}=-\frac{1}{\sqrt{\eta}} \cos \sigma+\frac{1}{\eta} \sin ^{2} \sigma+\cdots
$$

and the non-zero matrix elements

$$
V_{n, n}=\frac{1}{2 \eta}, \quad V_{n, n \pm 1}=-\frac{1}{2 \sqrt{\eta}}, \quad V_{n, n \pm 2}=-\frac{1}{4 \eta} .
$$

Thus

$$
X_{\psi}=\frac{1}{2 \eta} \sum_{n \in \mathbb{Z}} \frac{1}{n^{2}+\Omega^{2}}\left(1-\frac{1}{4} \frac{1}{(n+1)^{2}+\Omega^{2}}-\frac{1}{4} \frac{1}{(n-1)^{2}+\Omega^{2}}\right) .
$$

For the combination, we have

$$
\begin{aligned}
8 X_{\psi}-2 X_{\beta}- & X_{\phi}=-\frac{1}{\eta} \sum_{n \in \mathbb{Z}} \frac{1}{n^{2}+\Omega^{2}}\left(\frac{1}{(n+1)^{2}+\Omega^{2}}+\frac{1}{(n-1)^{2}+\Omega^{2}}\right) \\
& +\frac{2}{\eta} \sum_{n \in \mathbb{Z}} \frac{1}{n^{2}+\Omega^{2}}\left(\frac{1}{(n+2)^{2}+\Omega^{2}}+\frac{1}{(n-2)^{2}+\Omega^{2}}\right) .
\end{aligned}
$$

Evaluating the infinite sums over $n$, we find

$$
8 X_{\psi}-2 X_{\beta}-X_{\phi}=\frac{1}{\eta} \frac{2 \pi\left(2 \Omega^{2}-1\right) \operatorname{coth}(\pi \Omega)}{\Omega\left(\Omega^{2}+1\right)\left(4 \Omega^{2}+1\right)} .
$$

This is the same as Eq. (7.4) showing that the old-fashioned way of calculation is in perfect agreement with the the procedure adopted in this paper.

Comparing now this with the calculation in conformal gauge of [9], one can see that the difference is due to the second-order contribution of the $1 / \sqrt{\eta}$ term from the $\phi$ field. It is

$$
\frac{2}{\eta} \sum_{n \in \mathbb{Z}} \frac{1}{n^{2}+\Omega^{2}}\left(\frac{1}{(n+2)^{2}+\Omega^{2}}+\frac{1}{(n-2)^{2}+\Omega^{2}}\right)=\frac{2 \pi \operatorname{coth}(\pi \Omega)}{\Omega\left(\Omega^{2}+1\right)} .
$$

Now, reconsider equation (3.32) of [9] which reads

$$
\int_{\mathbb{R}} \mathrm{d} \Omega \sum_{n \in \mathbb{Z}}\left[\frac{2}{n^{2}+\Omega^{2}}-\frac{\mathrm{i} \Omega}{n^{2}+(\Omega+\mathrm{i})^{2}}+\frac{\mathrm{i} \Omega}{n^{2}+(\Omega-\mathrm{i})^{2}}\right]=0,
$$

upon doing a shift in $\Omega$ in the last two terms. Actually, one could perform first the sum over modes of the above integrand thus getting

$$
\sum_{n \in \mathbb{Z}}\left[\frac{2}{n^{2}+\Omega^{2}}-\frac{\mathrm{i} \Omega}{n^{2}+(\Omega+\mathrm{i})^{2}}+\frac{\mathrm{i} \Omega}{n^{2}+(\Omega-\mathrm{i})^{2}}\right]=\frac{2 \pi \operatorname{coth}(\pi \Omega)}{\Omega\left(\Omega^{2}+1\right)} .
$$

This means that avoiding the shifts in equation (3.32) of [9] one recovers full equality with the static gauge.

\section{References}

[1] Gubser S S, Klebanov I R and Polyakov A M 2002 A semi-classical limit of the gauge/string correspondence Nucl. Phys. B 63699 (arXiv:hep-th/0204051)

[2] de Vega H J and Egusquiza I L 1996 Planetoid string solutions in $3+1$ axisymmetric spacetimes Phys. Rev. D 547513 (arXiv:hep-th/9607056)

[3] Frolov S and Tseytlin A A 2002 Semiclassical quantization of rotating superstring in AdS(5) $\times S(5) J$. High Energy Phys. JHEP06(2002)007 (arXiv:hep-th/0204226)

[4] Beisert N, Eden B and Staudacher M 2007 Transcendentality and crossing J. Stat. Mech. 0701 P021 (arXiv:hep-th/0610251) 
[5] Basso B, Korchemsky G P and Kotanski J 2008 Cusp anomalous dimension in maximally supersymmetric Yang-Mills theory at strong coupling Phys. Rev. Lett. 100091601 (arXiv:0708.3933 [hep-th])

[6] Casteill P Y and Kristjansen C 2007 The strong coupling limit of the scaling function from the quantum string Bethe ansatz Nucl. Phys. B 7851 (arXiv:0705.0890 [hep-th])

Freyhult L and Kristjansen C 2006 A universality test of the quantum string Bethe ansatz Phys. Lett. B 638258 (arXiv:hep-th/0604069)

[7] Freyhult L and Zieme S 2009 The virtual scaling function of AdS/CFT Phys. Rev. D 79 105009 (arXiv:0901.2749 [hep-th])

Fioravanti D, Grinza P and Rossi M 2009 Beyond cusp anomalous dimension from integrability Phys. Lett. B 675137 (arXiv:0901.3161 [hep-th])

See also Bombardelli D, Fioravanti D and Rossi M 2009 Large spin corrections in $\mathcal{N}=4$ SYM sl(2): still a linear integral equation Nucl. Phys. B 810460 (arXiv:0802.0027 [hep-th])

[8] Basso B and Korchemsky G P 2007 Anomalous dimensions of high-spin operators beyond the leading order Nucl. Phys. B 7751 (arXiv:hep-th/0612247)

Korchemsky G 2007 Anomalous dimensions of high-spin operators beyond the leading order Talk at the 12th Claude Itzykson Meeting (Saclay, June 2007)

[9] Tirziu A and Tseytlin A A 2008 Quantum corrections to energy of short spinning string in AdS 5 Phys. Rev. D 78066002 (arXiv:0806.4758 [hep-th])

[10] Beccaria M, Forini V, Tirziu A and Tseytlin A A 2009 Structure of large spin expansion of anomalous dimensions at strong coupling Nucl. Phys. B 812144 (arXiv:0809.5234 [hep-th])

[11] Ambjorn J, Janik R A and Kristjansen C 2006 Wrapping interactions and a new source of corrections to the spin-chain/string duality Nucl. Phys. B 736288 (arXiv:hep-th/0510171)

[12] Schafer-Nameki S, Zamaklar M and Zarembo K 2006 How accurate is the quantum string Bethe ansatz? J. High Energy Phys. JHEP12(2006)020 (arXiv:hep-th/0610250)

[13] Bajnok Z, Janik R A and Lukowski T 2009 Four-loop twist two, BFKL, wrapping and strings Nucl. Phys. B 816376 (arXiv:0811.4448 [hep-th])

Lukowski T, Rej A and Velizhanin V N 2009 Five-loop anomalous dimension of twist-two operators arXiv:0912.1624

[14] Roiban R and Tseytlin A A 2009 Quantum strings in $\mathrm{AdS}_{5} \times S^{5}$ : strong-coupling corrections to dimension of Konishi operator J. High Energy Phys. JHEP11(2009)013 (arXiv:0906.4294 [hep-th])

[15] Kazakov V A, Marshakov A, Minahan J A and Zarembo K 2004 Classical/quantum integrability in AdS/CFT J. High Energy Phys. JHEP05(2004)024 (arXiv:hep-th/0402207)

Beisert N, Kazakov V A, Sakai K and Zarembo K 2006 The algebraic curve of classical superstrings on $\operatorname{AdS}(5) \times$ S(5) Commun. Math. Phys. 263659 (arXiv:hep-th/0502226)

[16] Gromov N 2009 Integrability in AdS/CFT correspondence: quasi-classical analysis J. Phys. A: Math. Theor. 42254004

Gromov N and Vieira P 2008 Complete one-loop test of AdS/CFT J. High Energy Phys. JHEP04(2008)046 (arXiv:0709.3487 [hep-th])

Beisert N and Freyhult L 2005 Fluctuations and energy shifts in the Bethe ansatz Phys. Lett. B 622343 (arXiv:hep-th/0506243)

[17] Gromov N 2009 Y-system and quasi-classical strings arXiv:0910.3608

[18] Beccaria M, Dunne G V, Forini V, Gromov N, Pawellek M and Tseytlin A (in preparation)

[19] Frolov S, Tirziu A and Tseytlin A A 2007 Logarithmic corrections to higher twist scaling at strong coupling from AdS/CFT Nucl. Phys. B 766232 (arXiv:hep-th/0611269)

[20] Fradkin E S and Tseytlin A A 1982 On quantized string models Ann. Phys. 143413

[21] Drukker N, Gross D J and Tseytlin A A 2000 Green-Schwarz string in $\operatorname{AdS}(5) \times S(5)$ : semiclassical partition function J. High Energy Phys. JHEP04(2000)021 (arXiv:hep-th/0001204)

[22] Schafer-Nameki S 2006 Exact expressions for quantum corrections to spinning strings Phys. Lett. B 639571 (arXiv:hep-th/0602214)

[23] Frolov S and Tseytlin A A 2003 Quantizing three-spin string solution in $\operatorname{AdS}(5) \times S(5) J$. High Energy Phys. JHEP07(2003)016 (arXiv:hep-th/0306130)

[24] Gromov N 2008 (unpublished)

[25] Kirsten K and McKane A J 2004 Functional determinants for general Sturm-Liouville problems J. Phys. A: Math. Gen. 374649 (arXiv:math-ph/0403050)

[26] Dunne G V and Feinberg J 1998 Self-isospectral periodic potentials and supersymmetric quantum mechanics Phys. Rev. D 571271 (arXiv:hep-th/9706012)

[27] Schafer-Nameki S and Zamaklar M 2005 Stringy sums and corrections to the quantum string Bethe ansatz J. High Energy Phys. JHEP10(2005)044 (arXiv:hep-th/0509096) 
[28] Dunne G V 2008 Functional determinants in quantum field theory J. Phys. A: Math. Theor. 41304006 (arXiv:0711.1178 [hep-th])

[29] Braden H W 1985 Periodic functional determinants J. Phys. A: Math. Gen. 182127

Braden H W 1987 Mass corrections to periodic solitons J. Math. Phys. 28929

[30] Whittaker E T and Watson G N 1927 A Course of Modern Analysis 4th edn (Cambridge: Cambridge University Press)

[31] Magnus W and Winkler S 1966 Hill's Equation (New York: Wiley)

[32] Dokshitzer Yu L and Marchesini G 2007 N = 4 SUSY Yang-Mills: three loops made simple(r) Phys. Lett. B 646189 (arXiv:hep-th/0612248)

[33] Beccaria M and Forini V 2009 Four loop reciprocity of twist two operators in $N=4$ SYM J. High Energy Phys. JHEP03(2009)111 (arXiv:0901.1256 [hep-th])

Beccaria M, Forini V and Macorini G 2010 Generalized Gribov-Lipatov reciprocity and AdS/CFT arXiv: 1002.2363

[34] Beccaria M and Tirziu A 2008 (unpublished)

[35] Kleinert H 2004 Path Integrals in Quantum Mechanics, Statistics, Polymer Physics, and Financial Markets (Singapore: World Scientific)

[36] Belokos E D, Bobenko A I, Enolskii V Z, Its A R and Matveev V B 1994 Algebro-Geometric Approach to Nonlinear Integrable Equations (Springer Series in Nonlinear Dynamics) (Berlin: Springer)

[37] Bena I, Polchinski J and Roiban R 2004 Hidden symmetries of the AdS(5) $\times$ S(5) superstring Phys. Rev. D 69046002 (arXiv:hep-th/0305116)

[38] Novikov S, Manakov S V, Pitaevsky L P and Zakharov V E 1984 Theory of Solitons: The Inverse Scattering Method (Contemporary Soviet Mathematics) (New York: Consultants Bureau) pp 276

[39] Arutyunov G, Frolov S and Staudacher M 2004 Bethe ansatz for quantum strings J. High Energy Phys. JHEP10(2004)016 (arXiv:hep-th/0406256)

[40] Vicedo B 2008 Semiclassical quantisation of finite-gap strings J. High Energy Phys. JHEP06(2008)086 (arXiv:0803.1605 [hep-th])

Vicedo B 2008 Finite-g strings arXiv:0810.3402 [hep-th]

[41] Gromov N and Vieira P 2008 The $\operatorname{AdS}(5) \times \mathrm{S}(5)$ superstring quantum spectrum from the algebraic curve Nucl. Phys. B 789175 (arXiv:hep-th/0703191)

[42] Gromov N, Schafer-Nameki S and Vieira P 2008 Efficient precision quantization in AdS/CFT J. High Energy Phys. JHEP12(2008)013 (arXiv:0807.4752 [hep-th])

[43] Beisert N and Tseytlin A A 2005 On quantum corrections to spinning strings and Bethe equations Phys. Lett. B 629102 (arXiv:hep-th/0509084)

[44] Hernandez R and Lopez E 2006 Quantum corrections to the string Bethe ansatz J. High Energy Phys. JHEP07(2006)004 (arXiv:hep-th/0603204)

[45] Park I Y, Tirziu A and Tseytlin A A 2005 Spinning strings in AdS(5) $\times$ S(5) one-loop correction to energy in SL(2) J. High Energy Phys. JHEP03(2005)013 (arXiv:hep-th/0501203)

[46] Gromov N and Vieira P 2008 Complete one-loop test of AdS/CFT J. High Energy Phys. JHEP04(2008)046 (arXiv:0709.3487 [hep-th]) 